Zoraida Violeta López Murgueytio

Estudo sobre a garantia de estabilidade em malha fechada com estimação algébrica de derivadas

São Paulo

2016 
Zoraida Violeta López Murgueytio

\title{
Estudo sobre a garantia de estabilidade em malha fechada com estimação algébrica de derivadas
}

\author{
Tese apresentada à Escola \\ Politécnica da Universidade de São \\ Paulo para obtenção do título de \\ Doutor em Ciências.
}

Área de concentração:

Engenharia de Sistemas

Orientador:

Prof. Dr. Paulo Sérgio Pereira da

Silva

São Paulo

2016 
Este exemplar foi revisado e corrigido em relação à versão original, sob responsabilidade única do autor e com a anuência de seu orientador.

São Paulo, de de

Assinatura do autor:

Assinatura do orientador:

Catalogação-na-publicação

Murgueytio, Zoraida Violeta López

Estudo sobre a garantia de estabilidade em malha fechada com estimação algébrica de derivadas / Z. V. L. Murgueytio -- versão corr. -- São Paulo, 2016. $112 \mathrm{p}$.

Tese (Doutorado) - Escola Politécnica da Universidade de São Paulo. Departamento de Engenharia de Telecomunicações e Controle.

1.Controle (teoria de sistemas e controle) 2.Estimação de derivadas 3.Sistemas não lineares 4.Levitação magnética I.Universidade de São Paulo. Escola Politécnica. Departamento de Engenharia de Telecomunicações e Controle II.t. 


\section{AGRADECIMENTOS}

A Deus, pela graça de ter me permitido concluir este trabalho.

Aos meus pais, Nadjy e Manuel, ao meu esposo Igor e ao meu filho César pelo carinho e apoio incondicional que sempre tive deles.

Ao meu orientador Prof. Dr. Paulo Sérgio Pereira da Silva, pelos tantos conselhos, paciência e dedicação, que me acompanhou durante o processo de elaboração da tese.

À CAPES, pelo financiamento da presente tese e à Escola Politécnica da Universidade de São Paulo, pela oportunidade de realização do curso de doutorado. 


\section{RESUMO}

A presente tese trata do estudo dos estimadores algébricos aplicados a sistemas de controle. Tais estimadores são filtros variantes no tempo que estimam as derivadas dos sinais de entrada e funcionam como uma alternatva ao uso de observadores. Este trabalho inclui uma contribuição teórica que permite determinar um limite superior do erro do estimador algébrico. Também, mostramos um resultado que garante estabilidade em malha fechada no contexto do princípio da separação para esta classe particular de sistemas. Um exemplo de levitação magnética baseado em linearização exata com estimadores algébricos de derivadas até segunda ordem implementados digitalmente é tratado com detalhe. Simulações em computador são apresentadas mostrando excelentes resultados.

Palavras-chaves: Estimadores algébricos, estabilidade, sistemas não lineares, levitação magnética. 


\begin{abstract}
This thesis deals with the algebraic estimators and their application in closed loop control systems. The algebraic estimators can be implemented as time-varying filters that produce an estimation of the derivatives of the input signal, and they can be an interesting alternative for substituting classical observers. This work includes a theoretic contribution that allows to compute a bound of the estimation error of the algebraic estimator. Furthermore it is shown that any derivative estimator that respects this bound will assure closed loop stability in the context of the separation principle, at least for a class of nonlinear systems. An example of magnetic levitation based on exact linearization and in closed loop with an estimator of the first and second derivatives is considered. A digital implementation of the algebraic estimation is also discussed, and simulations are presented, showing excellent results.
\end{abstract}

Keywords: Algebraic estimator, stability, nonlinear control, magnetic levitation. 


\section{Lista de símbolos}

$g$

$h$

$\mathcal{M}$

$\mathcal{N}$

$R_{k}$

$\hat{x}$

$\mathcal{K}$

V

$\lambda$

$\lambda_{n}$

M

$M_{r}$

K

$k_{d}$

$k_{m}$

G

$G_{\phi}$

$y_{\text {ref }}$

$f$

$M_{p}$

$k_{m p}$

$k_{d p}$

$G_{m a}$

$G_{m f}$

Aceleração da gravidade

período de reinicialização do estimador

máximo valor de $y^{(2)}$ em $[0, t]$

mínimo valor de $y^{(3)}$ em $[0, t]$

resto de Taylor

estado estimado

constante de Lypchitz

função de Lyapunov

distancia do solo ao mancal

altura de "contato parcial" com o solo

massa real de cada elemento (modelo flexível)

massa real do modelo rígido

constante da mola

constante do sensor de posição

constante de força mancal

gap desejado

gap inicial

trajetoria desejada

força externa desconhecida

valor presumido de $M_{r}$

valor presumido de $k_{m}$

valor presumido de $k_{d}$

função de transferencia em malha aberta

função de transferencia em malha fechada 


\section{Sumário}

1 INTRODUÇÃO 13

1.1 Organização do trabalho . . . . . . . . . . . . . . . . . 17

2 ESTIMADORES ALGÉBRICOS 18

2.1 Descrição do método algébrico . . . . . . . . . . . . . . . . 18

2.1.1 A ideia de base do método . . . . . . . . . . . . . . . . . . 19

2.2 Dinâmica do estimador de ordem dois . . . . . . . . . . . . . . . . 21

2.2.1 Análise do erro do filtro de segunda ordem . . . . . . . . . . . . . 24

2.3 Dinâmica do estimador de ordem três . . . . . . . . . . . . . . . 26

2.3.1 Análise da precisão do estimador algébrico de ordem três . . . . . . . . 31

2.3.2 Erro de estimação . . . . . . . . . . . . . . . . . 36

3 ESTABILIDADE EM MALHA FECHADA 38

3.1 Estabilidade em malha fechada para o filtro de segunda ordem . . . . . 39

3.2 Estabilidade em malha fechada para o filtro de terceira ordem . . . . . 43

3.2.1 Estabilidade exponencial para o filtro de terceira ordem . . . . . . . . 45

3.2.2 Estabilidade assintótica para o filtro de terceira ordem . . . . . . . . 46

3.3 Prova dos Teoremas . . . . . . . . . . . . . . . . . . . . 48

3.3.1 Considerações comuns aos teoremas 3 e 4 . . . . . . . . . . . . . . 48

3.3.2 Prova do teorema $3 \ldots \ldots \ldots \ldots$. . . . . . . . . . . . . . 51 
3.3 .3 Prova do Teorema $4 \ldots \ldots \ldots \ldots$. . . . . . . . . . . 54

3.4 Implementação sem reinicialização . . . . . . . . . . . . . . . . . 54

4 APLiCAÇÃo NO CONTROLE DE UM MANCAL MAGNETICO 60

4.1 Modelo do mancal magnético . . . . . . . . . . . . . . . . . . . 61

4.1 .1 Modelo rígido . . . . . . . . . . . . . . . . . . 61

4.1 .2 Modelo flexível . . . . . . . . . . . . . . . . . . . . 63

4.2 Linearização exata $(\mathrm{LE}) \ldots \ldots$. . . . . . . . . . . . . 65

4.3 Controle via Linearização Exata com Estimadores Algebricos (LEA) . . 74

4.4 Controle sem termo de gravidade nem de força externa . . . . . . . . . 75

4.5 Simulações do controle LE e LEA . . . . . . . . . . . . . . . . . 77

4.5 .1 Modelo flexível (sem ruído) . . . . . . . . . . . . 78

4.5.1.1 Simulações de desempenho sem ruído do modelo flexível . . . . . . . 78

4.5 .2 Modelo flexível (com ruído) . . . . . . . . . . . . . . 78

4.5.2.1 Simulações de desempenho com ruído do modelo flexível . . . . . . . 78

4.5.2.2 Erro de rastreamento do modelo flexível . . . . . . . . . . . . . . 80

4.5.2.3 Simulação focando o estimador do modelo flexível . . . . . . . . . . . 81

4.5.2.4 Esforço de controle do modelo flexível . . . . . . . . . . . . . . 82

4.5.3 Modelo Rígido (com ruído) . . . . . . . . . . . . . . . . . . . 83

4.5.3.1 Desempenho com ruído do modelo rígido . . . . . . . . . . . . 83

4.5.4 Simulações do controle da seção 4.4 . . . . . . . . . . . . . . . . . . . 84

4.5.4.1 Simulaçao do erro de rastreamento . . . . . . . . . . . . . . 85

4.5.4.2 Esforço de controle . . . . . . . . . . . . . . . . . . . . 86

5 CONSIDERAÇÕES FINAIS $\quad 90$ 
Referências Bibliográficas

A Artigo: Closed-loop performance analysis of algebraic estimators 95 


\section{Lista de Figuras}

3.1 Regra do Trapézio . . . . . . . . . . . . . . . 56

3.2 Implementação digital da integração pelo método trapezoidal . . 57

3.3 Diagrama de Blocos da Integral . . . . . . . . . . . . . . . 58

4.1 Modelo para controle do Mancal Magnético (Modelo Rígido) . . . . 62

4.2 Modelo para simulação do mancal magnético (Modelo flexível) . . 64

4.3 Gráfico do sinal de referência $y_{\text {ref }} \ldots \ldots$. . . . . . . . 66

4.4 Gráfico da derivada do sinal de referência $y_{r e f}^{(1)} \ldots \ldots$. . . . . . . . 67

4.5 Diagrama do sistema em malha fechada . . . . . . . . . . . 71

4.6 Gráfico do lugar das raizes . . . . . . . . . . . . . . . . . 72

4.7 Diagrama do sistema de controle em malha fechada . . . . . . . 73

4.8 Diagrama de estimação e controle em malha fechada ..... . 74

4.9 Diagrama de estimação e controle em malha fechada com ruido . . 75

4.10 Posição real utilizando o controlador LE e LEA. . . . . . . . . . . . 78

4.11 Velocidade real utilizando o controlador LE e LEA. . . . . . . . . . 79

4.12 Aceleração real utilizando o controlador LE e LEA . . . . . . . . 79

4.13 Posição real utilizando o controlador LE e LEA com ruído ... . 80

4.14 Velocidade real utilizando o controlador LE e LEA com ruído . . . 80

4.15 Aceleração real utilizando o controlador LE e LEA . . . . . . . . . 81

4.16 Zoom da aceleração real . . . . . . . . . . . . . . . . . . . 81 
4.17 Comparação entre os erros de rastreamento $z_{1}$ LE e LEA . . . . . . 82

4.18 Comparação entre os erros de rastreamento $z_{2}$ LE e LEA . . . . . . 82

4.19 Comparação entre os erros de rastreamento $z_{3}$ LE e LEA . . . . . . 83

4.20 Estado estimado e estado real (velocidade) . . . . . . . . . . . 83

4.21 Estado estimado e estado real (aceleração) . . . . . . . . . . . . . . 84

4.22 Comparação entre os esforços de controle LE e LEA . . . . . . . . . 84

4.23 Posição real do modelo rígido utilizando o controlador LE e LEA com ruído . . . . . . . . . . . . . . . . . . . 85

4.24 Velocidade real do modelo rígido utilizando o controlador LE e LEA. 85

4.25 Aceleração real do modelo rígido utilizando o controlador LE e LEA. 86

4.26 Posição real utilizando o controlador LE e LEA com ruído ...86

4.27 Velocidade real utilizando o controlador LE e LEA com ruído . . . 87

4.28 Aceleração real utilizando o controlador LE e LEA com ruído . . 87

4.29 Comparação entre os erros de rastreamento $z_{1}$ LE e LEA . . . . . . 88

4.30 Comparação entre os erros de rastreamento $z_{2}$ LE e LEA . . . . . . 88

4.31 Comparação entre os erros de rastreamento $z_{3}$ LE e LEA . . . . . . 89

4.32 Comparação entre os esforços de controle LE e LEA . . . . . . . . . 89 


\section{Lista de Tabelas}

4.1 Valores dos dados utilizados na simulação . . . . . . . . . . . 63

4.2 Modelo rígido e flexível . . . . . . . . . . . . . . . . . 65 


\section{Capítulo 1}

\section{INTRODUÇÃO}

O estudo de controle moderno está baseado no conceito de estado (Ogata 1982), introduzido nos trabalhos de Poincare no final do seculo XIX, o qual mais tarde seria conhecido como método de espaço de estado. Este conceito também foi utilizado nos trabalhos pioneiros de Kalmam e Luenberger no controle por realimentação de estado, tema que até hoje continua sendo objeto de pesquisa. A teoria de estimação de estados e os observadores assintóticos em particular o teorema da separação determinística (Chen 1999) têm sido fundamentais no projeto de controle de sistemas lineares. Embora o mesmo resultado não seja verdadeiro para sistemas não lineares, a literatura já tem apresentado adaptações e generalizações para o caso não linear.

O controle de sistemas não lineares na topologia observador-controlador é um tema muito atual de pesquisa (Cunha et al. 2005, Ahrens e Khalil 2009, Oliveira, Peixoto e Hsu 2010, Oliveira, Peixoto e Hsu 2011). Tal topologia tem relação direta com diferentes versões do teorema da separação para sistemas não lineares (Atassi e Khalil 1999, Atassi e Khalil 2000, Atassi e Khalil 2001) e pode 
ser generalizada para problemas de rastreamento.

Este trabalho de pesquisa se centra no estudo de uma abordagem alternativa para o projeto de estimação de estados. Esta classe de estimadores é denominada de estimadores algébricos, os quais não tem natureza assintótica. Várias pesquisas mostraram que podem ser substituídos os observadores tradicionais pelos estimadores de derivadas (Plestan e Grizzle 1999, Diop et al. 1994, Diop, Grizzle e Chaplais 2000, Ibrir e Diop 2004, Levant 2003).

Um resultado importante no controle não linear mostra que um sistema desse tipo com estado $x$, entrada $y$ definido da seguinte forma:

$$
\begin{aligned}
\dot{x}(t) & =f(x(t), u(t)) \\
y & =h(x(t))
\end{aligned}
$$

é observável se e somente se seu estado puder ser determinado a partir do conhecimento da entrada, da saída e suas derivadas. Em outras palavras, existe uma aplicação $\psi$ tal que (Diop e Fliess 1991):

$$
x=\psi\left(u, \dot{u}, \ldots, u^{(\mu)}, y, \dot{y}, \ldots, y^{(\eta)}\right)
$$

isso mostra que o problema de estimação de estado está diretamente relacionado ao problema de estimação de derivadas.

Trabalhos desenvolvidos por (Fliess e Sira-Ramirez 2004, Fliess e Sira-Ramirez 2004) 
sobre o método algébrico de estimação de derivadas usam as propriedades do cálculo operacional na construção do estimador de derivadas de ordem arbitrária de um sinal dado $y(t)$. Outras pesquisas também mostraram que:

- O estimador pode ser implementado por meio de um filtro linear variante no tempo (Reger, Mai e Sira-Ramirez 2006, Reger, Sira-Ramirez e Fliess 2005)

- Esses filtros também podem ser implementados em computador depois de uma adequada discretização como visto em (Novaes e Silva 2009, Novaes 2010) e que as propriedades fundamentais desses estimadores são mantidas para suas implementações digitais.

- Resultados bem sucedidos são mostrados em (Fliess e Sira-Ramirez 2004) no controle de alguns sistemas não lineares, onde os estados estimados são obtidos de fórmulas algébricas, as quais fornecem uma boa aproximação em tempo real, mesmo num ambiente ruidoso.

Neste trabalho o estimador a ser estudado utilizará a abordagem algébrica para a estimação das derivadas. Apresentaremos inicialmente o estimador algébrico de ordem dois, que é o mais simples desta familia, sendo que ele estima apenas a primeira derivada do sinal dado. Depois disso apresentaremos o estimador algébrico de ordem superior (ordem três) que calcula a primeira e a segunda derivadas do sinal. Também será feita a análise da precisão de ambos estimadores (ordem dois e três), onde se estabelecem fórmulas que permitem a previsão de um limite para o erro de cada estimador. Estas fórmulas são posteriormente utilizadas no estudo da estabilidade em malha fechada, fornecendo um resultado que pode ser considerado semelhante ao teorema de separação.

Um trabalho mais recente (Mboup, Join e Fliess 2009) mostra que os estimadores algébricos podem ser implementados sem a necesidade de re-inicialização. O 
presente trabalho começa utilizando a versão dos filtros variantes no tempo que ainda exigem re-incialização periódica para garantir a precisão da estimação. As demostrações dos teoremas são baseadas nesta versão dos algoritmos, mas uma pequena adaptação dessas demonstrações poderia ser feita para considerar as versões mais recentes dos estimadores, que são também discutidas na presente tese.

$\mathrm{Na}$ dissertação de mestrado (Murgueytio 2011) foram realizadas muitas simulações comparativas de desempenho dos estimadores algébricos num estudo de caso (o pêndulo invertido) onde é mostrado o compromisso entre a precisão da estimação, que implica na robustez em malha fechada e na imunidade a ruído (veja também o artigo (Murgueytio, Silva e Novaes 2013) anexado na presente tese). As comparações foram feitas entre os estimadores algébricos e as medidas perfeitas, de modo que as comparações dos estimadores algébricos com os observadores tradicionais não foram discutidas naqueles trabalhos.

O objetivo deste trabalho não é a avaliação comparativa de desempenho dos estimadores algébricos em nenhum dos casos (isto é, nem a comparação com observadores tradicionais, nem a comparação com a medida perfeita) pois isso é assunto que pode ser encontrado na literatura. O objetivo deste trabalho é a prova de estabilidade em malha fechada. Ressaltamos que a natureza dessas demostrações é diferente das provas dos "teoremas da separação", pois os mesmos incluem a dinâmica do observador, de maneira que a estabilidade se refere a um sistema estendido. No nosso caso, os estimadores são praticamente instantâneos e ainda todas as operações dos estimadores algébricos são obtidas por integrações em janelas finitas. O observador, pelo menos o linear, é um sistema dinâmico cuja saída seria dada por uma integral de convolução: 


$$
\hat{x}=\int_{t_{0}}^{t} H(t-\tau)\left(\begin{array}{l}
u(\tau) \\
y(\tau)
\end{array}\right) d \tau
$$

que é uma integral cuja janela $\left[t_{0}, t\right]$ não é limitada. Para estimadores algébricos, a integral em janela finita de sinais limitados é limitada. Portanto, podemos estudar a estabilidade do sistema em malha fechada com os estimadores algébricos sem incluir a dinâmica dos estimadores, mas apenas mostrando a convergência do estado do sistema.

\subsection{Organização do trabalho}

O capítulo 2 apresenta o método algébrico de forma sucinta, a ideia base do método é apresentada, obtendo-se uma expressão que fornece um sistema triangular de equações de onde podem ser deduzidas as fórmulas explícitas que aproximam as derivadas no tempo dos sinais. Neste capítulo são mostradas a dinâmica dos estimadores de ordem dois e três, assim como a análise do erro cometido por ambos estimadores.

O capítulo 3 estuda a estabilidade em malha fechada para esta classe de estimadores, apresentando os resultados para os estimadores de ordem dois e três.

Uma aplicação para ilustrar o método algébrico estudado é dada no capítulo 4, onde estudamos uma lei de controle estabilizante para um sistema de levitação magnética.

O capítulo 5 apresenta as conclusões do trabalho. 


\section{Capítulo 2}

\section{ESTIMADORES ALGÉBRICOS}

\subsection{Descrição do método algébrico}

O método algébrico de estimação em sistemas de controle proposto por Fliess e co-autores tem contribuído no desenvolvimento de uma série de resultados interessantes na estimação de estados, identificação paramétrica e diagnóstico de falhas entre outros.

Uma abordagem interessante proposta por (Diop e Fliess 1991, Diop et al. 1994) e (Diop, Grizzle e Chaplais 2000), propõe uma metodologia de estimação das derivadas de um sinal dado. Neste trabalho, nosso interesse é numa abordagem essencialmente focada em técnicas de cálculo operacional (transformadas de Laplace) para a estimação de estados, baseada no "método algébrico diferencial" apresentado por (Fliess e Sira-Ramirez 2004) e (Fliess e Sira-Ramirez 2004). O esquema de estimação proposto é baseado na expansão em série de Taylor truncada de um sinal analítico, e sua representação como uma cadeia de integradores. Utilizando o cálculo operacional, eliminamos a influência dos valores iniciais e obtemos um sis- 
tema triangular de equações, que permitem resolver o problema para as respectivas derivadas dos sinais de tempo até uma ordem desejada. Como essa aproximação é válida somente num determinado intervalo de tempo, uma reinicialização é proposta para garantir a exatidão do procedimento. Por tal razão, vão se reiniciar os cálculos após um intervalo de tempo pequeno de comprimento fixo.

\subsubsection{A ideia de base do método}

Seja $y(t)$ um sinal $C^{\infty}$ arbitrária, é proposto um método para obter aproximações para as derivadas de tempo do sinal $y(t)$. Sabemos que o valor do sinal $y(t)$ em $t>0$ é aproximado pela clássica expansão em série de Taylor truncada:

$$
\tilde{y}(t)=\sum_{j=1}^{k} \frac{1}{(j-1) !} y^{(j-1)}(0) t^{(j-1)}
$$

onde $y^{(k)}(0)$ representa a k-ésima derivada no tempo de $y(t)$ avaliada no instante $t=0$.

Obviamente, quanto mais alto o valor de $k$, menor é a diferença $y(t)-\tilde{y}(t)$, e isso pode ser deduzido da fórmula do erro de truncamento da fórmula de Taylor, por exemplo, a fórmula do Resto de Lagrange:

$$
R_{k}(t) \leq \frac{1}{k !} \sup _{\tau \in[0, t]}\left|y^{(k)}(\tau)\right|
$$

Note que, se $y(t)$ for analítica num intervalo $[0, h]$, teremos que $\lim _{k \rightarrow \infty} R_{k}(t)$ será nulo uniformemente em $t$ para $t \in[0, h]$, pelo menos para $h$ menor que o raio de 
convergência da série de Taylor.

Observe agora que a série de Taylor truncada (2.1) pode ser identificada com a resposta de um sistema linear homogêneo com um conjunto de condições iniciais representado pelo valor inicial do sinal calculado no instante $t=0$, e ainda as primeiras $k-1$ derivadas do sinal $y(t)$, também calculadas no instante $t=0$. Nesse caso podemos escrever:

$$
\begin{aligned}
& \tilde{y}^{(k)}(t)=0 \\
& \tilde{y}^{(j)}(0)=y^{(j)}(0), j=0, \ldots, k-1
\end{aligned}
$$

Na notação de cálculo operacional, a aproximação $\tilde{y}(s)$ do sinal $y(t)$ é representada por

$$
s^{k} \tilde{y}(s)-\sum_{j=1}^{k} s^{k-j} \tilde{y}^{(j-1)}(0)=0
$$

A equação anterior pode ser obtida através do formalismo do cálculo operacional, mas também é obtida aplicando-se a transformada de Laplace $^{1}$ na equação (2.2). A k-ésima derivação da equação (2.4) com respeito ao operador $s$ produz a seguinte expressão:

$$
\frac{d^{k}}{d s^{k}}\left(s^{k} \tilde{y}(s)\right)=0
$$

que é independente de todas as condições iniciais desconhecidas. Multiplicando a esquerda por $s^{-j}$, a equação (2.5) para $j=k-1, \ldots, 2,1$, obtemos

$$
s^{-j} \frac{d^{k}}{d s^{k}}\left(s^{k} \tilde{y}(s)\right)=0
$$

Essa é uma expressão importante, que fornece um sistema triangular de

\footnotetext{
${ }^{1}$ Note que $\mathscr{L}\left(\tilde{y}^{k}(t)\right)=s^{k} \tilde{y}(s)-\sum_{j=1}^{k} s^{k-j} \tilde{y}^{j-1}(0)$
} 
equações lineares a partir do qual as derivadas no tempo do sinal aproximado $\tilde{y}$ de ordem entre 1 e $k-1$ podem ser calculadas. A ideia então é adotar essas funções obtidas como as aproximações das derivadas de tempo do sinal original $y(t)$, retransformando para o domínio do tempo (2.6), e obtendo assim fórmulas explícitas para aproximar as diferentes derivadas de tempo de $y(t)$. Na seção seguinte estudaremos o caso particular em que $k=2$, mostrando como (2.6) pode ser resolvida.

\subsection{Dinâmica do estimador de ordem dois}

Até o final deste trabalho o valor real do sinal será denotado por $y(t)$, o valor da sua aproximação polinomial de grau 1 será denotado por $\tilde{y}(t)$ dado por

$$
\tilde{y}=y(0)+y^{(1)}(0) t
$$

A estimativa de derivada dada pelo estimador algébrico será denotada por $\widehat{\dot{y}}(t)$. A derivada (verdadeira) do sinal será denotada por $\dot{y}(t)$, ou ainda por $y^{(1)}(t)$.

Considere-se a aproximação de primeira ordem, no tempo $t=0$, isto é, o truncamento da série de Taylor para $k=2$. Substituindo $k=2$ na equação $(2.1)$ temos:

$$
\tilde{y}(t)=\sum_{j=1}^{2} \frac{1}{(j-1) !} y^{(j-1)}(0) t^{j-1}
$$

Note que $\tilde{y}(t)$ satisfaz a equação diferencial linear homogênea e invariante no tempo

$$
\tilde{y}^{(2)}(t)=0
$$

Pode-se expressar esta equação na notação de cálculo operacional, como visto na 
equação (2.5), obtendo-se

$$
\frac{d^{2}}{d s^{2}}\left(s^{2} \tilde{y}(s)\right)=0
$$

Substituindo os valores $k=2$ e $j=1$, na equação (2.6), obtemos:

$$
s^{-1} \frac{d^{2}}{d s^{2}}\left[s^{2} \tilde{y}(s)\right]=0
$$

De (2.8) teremos

$$
s^{-1} \frac{d}{d s}\left[\frac{d}{d s}\left(s^{2} \tilde{y}(s)\right)\right]=0
$$

e assim:

$$
2 s^{-1} \tilde{y}(s)+4 \frac{d \tilde{y}(s)}{d s}+s \frac{d^{2} \tilde{y}(s)}{d s^{2}}=0
$$

Usando as propriedades básicas das transformadas de Laplace, a equação (2.9) no domínio do tempo é expressa da seguinte forma:

$$
2\left(\int_{0}^{t} \tilde{y}(\sigma) d \sigma\right)-4 t \tilde{y}(t)+\frac{d}{d t}\left[t^{2} \tilde{y}(t)\right]=0
$$

Note que

$$
\frac{d}{d t}\left[t^{2} \tilde{y}(t)\right]=2 t \tilde{y}(t)+t^{2} \tilde{y}^{(1)}(t)
$$

Voltando à equação (2.10) e substituindo a expressão anterior, obtemos:

$$
\left.2\left(\int_{0}^{t} \tilde{y}(\sigma) d \sigma\right)-2 t \tilde{y}(t)+t^{2} \tilde{y}^{(1)}(t)\right)=0
$$

Resolvendo para $\dot{\tilde{y}}^{(1)}$, podemos escrever:

$$
\tilde{y}^{(1)}(t)=\frac{1}{t^{2}}\left[-2 \int_{0}^{t} \tilde{y}(\sigma) d \sigma+2 t \tilde{y}(t)\right]
$$


A ideia de implementação do estimador é substituir, na equação anterior, $\tilde{y}$ por $y$, obtendo a seguinte fórmula aproximada:

$$
\widehat{\dot{y}}(t)=\frac{1}{t^{2}}\left[-2 \int_{0}^{t} y(\sigma) d \sigma+2 t y(t)\right] .
$$

para qualquer $t \geq \epsilon>0$ a expressão da derivada no tempo de primeira ordem de $y(t)$ fornece uma aproximação adequada. No entanto, durante o intervalo inicial $[0, \epsilon]$ podemos ter uma indeterminação no valor da derivada calculada $\dot{\tilde{y}}(t)$. O valor de $\epsilon$ é escolhido por razões de precisão computacional da divisão por um número $t^{2}$ muito pequeno. Por esta razão, pode-se substituir o valor de $\dot{y}$ por um valor constante arbitrário $c$ que em geral é escolhido como o valor da última estimação, se esse valor estiver disponível.

Para que a fórmula de $\dot{y}$ dada em (2.11) mantenha sua precisão na aproximação é necessário reinicializar os cálculos em algum tempo finito $t_{k}>0$, isto é, escolher um intervalo de tempo cujo comprimento $h$ pode ser fixado arbitrariamente pequeno, ver (Murgueytio, Silva e Novaes 2013). Assim assumimos a validade da fórmula (2.11), como é visto na equação (2.12):

$$
\widehat{\dot{y}}(t)=\frac{\left[-2 \int_{t_{k}}^{t} y(\sigma) d \sigma+2\left(t-t_{k}\right) y(t)\right]}{\left(t-t_{k}\right)^{2}}
$$

Na equação acima, $\widehat{\dot{y}}(t)$ denota a estimativa de $\dot{y}(t)$. Assim podem ser obtidos resultados precisos de estimação em todo instante de tempo $t \geq 0$, com a exceção dos instantes iniciais $t \in[0, \epsilon)$. 


\subsubsection{Análise do erro do filtro de segunda ordem}

Faremos a análise de erro para a fórmula (2.12) para $t \in[\epsilon, h]$ onde $\epsilon>0$ é suficientemente grande para evitar a singularidade da divisão por $t^{2}$, e $h$ é o período escolhido de reinicialização do estimador algébrico.

Seja $y:[0, h] \rightarrow \mathbb{R}$ um sinal de classe $C^{2}$. Seja

$$
\tilde{y}(t)=y(0)+y^{(1)}(0) t
$$

a aproximação de Taylor de primeira ordem de $y(t)$. Nesta análise, $\mathcal{M}(t)$ será o máximo valor que $y^{(2)}$ assume no intervalo compacto $[0, t]$, isto é

$$
\mathcal{M}(t)=\sup _{\tau \in[0, t]}\left|y^{(2)}(\tau)\right|
$$

Lembremos que

$$
y(t)=\tilde{y}(t)+R_{2}(t)
$$

onde $R_{2}(t)$ é o resto de Taylor. A fórmula de Lagrange do resto de Taylor nos fornece (vide (Lima 1981)):

$$
\left|R_{2}(t)\right| \leq \mathcal{M}|t|^{2} / 2
$$

Derivando a penúltima equação em ambos os lados, podemos escrever

$$
y^{(1)}(t)=\tilde{y}^{(1)}(t)+R_{2}^{(1)}(t)
$$

Como $\tilde{y}^{(1)}(t)=y^{(1)}(0)$, segue-se que

$$
R_{2}^{(1)}(t)=y^{(1)}(t)-y^{(1)}(0)
$$


Pelo Teorema do Valor Médio (vide (Lima 1981, p. 89)), existe $\xi \in[0, t]$ tal que $y^{(1)}(t)-y^{(1)}(0)=y^{(2)}(\xi)(t-0)$. Portanto

$$
\left|R_{2}^{(1)}(t)\right| \leq \mathcal{M}|t|
$$

Substituindo $y=\tilde{y}+R_{2}$ em (2.11), obtemos

$$
\begin{aligned}
\widehat{\dot{y}}(t)= & \frac{1}{t^{2}}\left[-2 \int_{0}^{t} \tilde{y}(\sigma) d \sigma+2 t \tilde{y}(t)\right]+ \\
& \frac{1}{t^{2}}\left[-2 \int_{0}^{t} R_{2}(\sigma) d \sigma+2 t R_{2}(t)\right] .
\end{aligned}
$$

Lembremos da seção anterior que $\tilde{y}$ é sempre solução de ${ }^{2}$

$$
\tilde{y}^{(1)}(t)=\frac{1}{t^{2}}\left[-2 \int_{0}^{t} \tilde{y}(\sigma) d \sigma+2 t \tilde{y}(t)\right] .
$$

Defina

$$
\Delta(t)=\frac{1}{t^{2}}\left[-2 \int_{0}^{t} R_{2}(\sigma) d \sigma+2 t R_{2}(t)\right]
$$

Então, (2.11) fornece

$$
\widehat{\dot{y}}(t)=\tilde{y}^{(1)}(t)+\Delta(t)=\tilde{y}^{(1)}(0)+\Delta(t)
$$

Portanto o erro cometido na estimação será

$$
\begin{aligned}
e(t) & =\widehat{\dot{y}}(t)-y^{(1)}(t) \\
& =\tilde{y}^{(1)}(0)+\Delta(t)-\tilde{y}^{(1)}(0)-R_{2}^{(1)}(t) \\
& =\Delta(t)-R_{2}^{(1)}(t)
\end{aligned}
$$

Como já temos um limite superior para $R_{2}^{(1)}(t)$, resta calcular um limite superior

\footnotetext{
${ }^{2}$ Isto é equivalente a dizer que a estimativa é exata para sinais polinomiais de grau 1.
} 
para $\Delta(t)$. Para isso note que, usando (2.14), a desigualdade triangular, e o fato de que $\left|\int_{0}^{t} f(\sigma) d \sigma\right| \leq \int_{0}^{t}|f(\sigma)| d \sigma$ para toda função contínua $f$, segue-se que:

$$
\begin{aligned}
|\Delta(t)| & =\left|\frac{1}{t^{2}}\left[-2 \int_{0}^{t} R_{2}(\sigma) d \sigma+2 t R_{2}(t)\right]\right| \\
& \leq \frac{1}{t^{2}}\left\{\left|2 \int_{0}^{t} \mathcal{M} \frac{\sigma^{2}}{2} d \sigma\right|+\mathcal{M}\left|t^{3}\right|\right\} \\
& \leq \frac{4}{3}|t| \mathcal{M}
\end{aligned}
$$

Sendo assim, de (2.13), um limite superior para o erro da estimativa dentro do intervalo $[0, t]$ será:

$$
\sup _{\tau \in[0, t]}|e(t)| \leq \frac{7}{3}|t| \sup _{\tau \in[0, t]}\left|y^{(2)}(t)\right|
$$

em particular, como os estimadores são reinicializados a cada $h$ segundos, um limite de erro para o estimador (2.12) será

$$
\sup _{\tau \in[0, h]}|e(t)| \leq \frac{7}{3}|h| \sup _{\tau \in[0, h]}\left|y^{(2)}(t)\right|
$$

Vemos que o máximo erro cometido é proporcional à máxima derivada segunda do sinal $y(t)$ e ao intervalo de reset $h$ do Estimador Algébrico.

\subsection{Dinâmica do estimador de ordem três}

O procedimento para obter o estimador de ordem três é muito similar ao que foi feito para o estimador de ordem dois.

Seja $y(t)$ o valor real do sinal e $\tilde{y}(t)$ é o valor de sua aproximação polinomial de 
segunda ordem, a qual é denotada da seguinte forma:

$$
\tilde{y}=y(0)+y^{(1)}(0) t+\frac{1}{2 !} y^{(2)}(0) t^{2}
$$

A estimativa da derivada será $\hat{\dot{y}}(t)$ e a derivada (verdadeira) será denotada por $\dot{y}(t)$ ou $y^{(1)}(t)$, a segunda derivada será denotada por $\ddot{y}(t)$ ou $y^{(2)}(t)$ e a estimativa da segunda derivada será $\hat{\ddot{y}}(t)$.

Considere-se a aproximação de segunda ordem, isto é, o truncamento da série de Taylor para $k=3$, teremos:

$$
\tilde{y}(t)=\sum_{j=1}^{3} \frac{1}{(j-1) !} y^{(j-1)}(0) t^{j-1}
$$

Onde $\tilde{y}(t)$ satisfaz a equação diferencial linear homogênea e invariante no tempo

$$
\tilde{y}^{(3)}(t)=0 \text {. }
$$

Expressando essa equação na notação de cálculo operacional, obtemos:

$$
\frac{d^{3}}{d s^{3}}\left(s^{3} \tilde{y}(s)\right)=0
$$

Substituindo os valores $k=3$ e $j=1,2$ na equação (2.6). Fazendo para $k=3$ e $j=2$, obtemos

$$
s^{-2} \frac{d^{3}}{d s^{3}}\left[s^{3} \tilde{y}(s)\right]=0
$$


De (2.20) temos

$$
s^{-2} \frac{d}{d s}\left[\frac{d}{d s}\left[\frac{d}{d s}\left(s^{3} \tilde{y}(s)\right)\right]\right]=0
$$

e assim:

$$
6 s^{-2} \tilde{y}(s)+18 s^{-1} \frac{d \tilde{y}(s)}{d s}+9 \frac{d^{2} \tilde{y}(s)}{d s^{2}}+s \frac{d^{3} \tilde{y}(s)}{d s^{3}}=0
$$

Utilizando as propriedades das transformadas de Laplace na equação (2.21), obtemos a seguinte expressão no domínio do tempo

$$
6\left(\int_{0}^{t} \int_{0}^{\sigma_{1}} \tilde{y}\left(\sigma_{2}\right) d \sigma_{2} \sigma_{1}\right)-18\left(\int_{0}^{t} \sigma_{1} \tilde{y}\left(\sigma_{1}\right) d \sigma_{1}\right)+9 t^{2} \tilde{y}(t)-\frac{d}{d t}\left[t^{3} \tilde{y}(t)\right]=0
$$

Note que

$$
\frac{d}{d t}\left[t^{3} \tilde{y}(t)\right]=3 t^{2} \tilde{y}(t)+t^{3} \dot{\tilde{y}}(t)
$$

substituindo esta expressão na equação (2.22), obtemos:

$$
6\left(\int_{0}^{t} \int_{0}^{\sigma_{1}} \tilde{y}\left(\sigma_{2}\right) d \sigma_{2} \sigma_{1}\right)-18\left(\int_{0}^{t} \sigma_{1} \tilde{y}\left(\sigma_{1}\right) d \sigma_{1}\right)+6 t^{2} \tilde{y}(t)-t^{3} \dot{\tilde{y}}(t)=0
$$

Resolvendo para $\dot{\tilde{y}}$ : 


$$
\dot{\tilde{y}}(t)=\frac{1}{t^{3}}\left[6\left(\int_{0}^{t} \int_{0}^{\sigma_{1}} \tilde{y}\left(\sigma_{2}\right) d \sigma_{2} \sigma_{1}\right)-18\left(\int_{0}^{t} \sigma_{1} \tilde{y}\left(\sigma_{1}\right) d \sigma_{1}\right)+6 t^{2} \tilde{y}(t)\right]
$$

Similarmente como foi considerado para a dinâmica do estimador de segunda ordem, neste caso também substituimos $\tilde{y}$ por $y$, e obtemos a seguinte fórmula aproximada:

$$
\widehat{\dot{y}}(t)=\frac{1}{t^{3}}\left[6\left(\int_{0}^{t} \int_{0}^{\sigma_{1}} y\left(\sigma_{2}\right) d \sigma_{2} \sigma_{1}\right)-18\left(\int_{0}^{t} \sigma_{1} y\left(\sigma_{1}\right) d \sigma_{1}+6 t^{2} y(t)\right]\right.
$$

Agora considerando os valores $k=3$ e $j=1$ na equação (2.6), obtemos:

$$
s^{-1} \frac{d^{3}}{d s^{3}}\left(s^{3} \tilde{y}(s)\right)=0
$$

Analogamente ao caso anterior, obteremos a seguinte expressão:

$$
6 s^{-1} \tilde{y}(s)+18 \frac{d \tilde{y}(s)}{d s}+9 s \frac{d^{2} \tilde{y}(s)}{d s^{2}}+s^{2} \frac{d^{3} \tilde{y}(s)}{d s^{3}}=0
$$

Utilizando as propriedades das transformadas de Laplace e aplicando na equação $(2.25)$

$$
6\left(\int_{0}^{t} \tilde{y}\left(\sigma_{1}\right) d \sigma_{1}\right)-18 t \tilde{y}(t)+9 \frac{d}{d t}\left[t^{2} \tilde{y}(t)\right]-\frac{d^{2}}{d t^{2}}\left[t^{3} \tilde{y}(t)\right]=0
$$

Note-se que 
- $\frac{d}{d t}\left[t^{2} \tilde{y}(t)\right]=2 t \tilde{y}(t)+t^{2} \dot{\tilde{y}}(t)$

- $\frac{d^{2}}{d t^{2}}\left[t^{3} \tilde{y}(t)\right]=6 t \tilde{y}(t)+6 t^{2} \dot{\tilde{y}}(t)+t^{3} \ddot{\tilde{y}}(t)$

Substituindo as expressões anteriores na equação (2.26), temos:

$$
6\left(\int_{0}^{t} \tilde{y}\left(\sigma_{1}\right) d \sigma_{1}\right)-6 t \tilde{y}(t)+3 t^{2} \dot{\tilde{y}}(t)-t^{3} \ddot{\tilde{y}}(t)=0
$$

Resolvendo para $\ddot{\tilde{y}}$, teremos:

$$
\ddot{\tilde{y}}(t)=\frac{1}{t^{3}}\left[6 \left(\int_{0}^{t} \tilde{y}\left(\sigma_{1}\right) d \sigma_{1}+3 t^{2} \dot{\tilde{y}}(t)-6 t \tilde{y}(t)\right.\right.
$$

Substituindo $\tilde{y}$ por $y$ na equação anterior, obtemos:

$$
\widehat{\ddot{y}}(t)=\frac{1}{t^{3}}\left[6\left(\int_{0}^{t} y(\sigma) d \sigma\right)+3 t^{2} \dot{y}(t)-6 t y(t)\right]
$$

Observe-se que a equação (2.28) é não implementavel porque usa $\dot{y}$ desconhecido. Em resumo teremos as formulas das aproximações dadas pelas equações (2.23) e (2.28):

$$
\begin{gathered}
\widehat{\dot{y}}(t)=\frac{1}{t^{3}}\left[6\left(\int_{0}^{t} \int_{0}^{\sigma_{1}} y\left(\sigma_{2}\right) d \sigma_{2} \sigma_{1}\right)-18\left(\int_{0}^{t} \sigma_{1} y\left(\sigma_{1}\right) d \sigma_{1}+6 t^{2} y(t)\right]\right. \\
\widehat{\ddot{y}}(t)=\frac{1}{t^{3}}\left[6\left(\int_{0}^{t} y(\sigma) d \sigma\right)+3 t^{2} \dot{y}(t)-6 t y(t)\right]
\end{gathered}
$$




\subsubsection{Análise da precisão do estimador algébrico de ordem} três

Seja $y:[0, h] \rightarrow \mathbb{R}$ um sinal de classe $C^{3}$. E seja

$$
\tilde{y}(t)=y(0)+y^{(1)}(0) t+\frac{y^{(2)}(0)}{2 !} t^{2}
$$

a aproximação de Taylor de segunda ordem de $y(t)$. Nesta análise, $\mathcal{N}(t)$ será o máximo valor que $y^{(3)}$ assume no intervalo compacto $[0, t]$, isto é

$$
\mathcal{N}(t)=\sup _{\tau \in[0, t]}\left|y^{(3)}(\tau)\right|
$$

Lembremos que

$$
y(t)=\tilde{y}(t)+R_{3}(t)
$$

onde $R_{3}(t)$ é o resto de Taylor.

A fórmula de Lagrange do resto de Taylor nos fornece

$$
\left|R_{3}(t)\right| \leq \mathcal{N}|t|^{3} / 3 \text { ! }
$$

Derivando a equação (2.32) em ambos os lados:

$$
y^{(1)}(t)-\tilde{y}^{(1)}(t)=R_{3}^{(1)}(t)
$$




$$
\begin{gathered}
y^{(1)}(t)-\frac{d}{d t}\left[y(0)+y^{(1)} t+\frac{y^{(2)}(0)}{2} t^{2}\right]=R_{3}^{1}(t) \\
y^{(1)}(t)=y^{(1)}(0)+y^{(2)}(0) t+R_{3}^{(1)}(t)
\end{gathered}
$$

Utilizando a fórmula de Lagrange do resto de Taylor, obtemos:

$$
\left|R_{3}^{(1)}(t)\right| \leq \frac{1}{2}\left|y^{(3)}(0)\right||t|^{2}
$$

Logo, substituindo $y=\tilde{y}+R_{3}$ na equação (2.29):

$$
\begin{array}{r}
\widehat{\dot{y}}(t)=\frac{1}{t^{3}}\left[6\left(\int_{0}^{t} \int_{0}^{\sigma_{1}} \tilde{y}\left(\sigma_{2}\right) d \sigma_{2} \sigma_{1}\right)-18\left(\int_{0}^{t} \sigma_{1} \tilde{y}\left(\sigma_{1}\right) d \sigma_{1}+6 t^{2} \tilde{y}(t)\right]+\right. \\
\frac{1}{t^{3}}\left[6\left(\int_{0}^{t} \int_{0}^{\sigma_{1}} R_{3}\left(\sigma_{2}\right) d \sigma_{2} \sigma_{1}\right)-18\left(\int_{0}^{t} \sigma_{1} R_{3}\left(\sigma_{1}\right) d \sigma_{1}+6 t^{2} R_{3}(t)\right]\right.
\end{array}
$$

Lembremos da seção anterior que $\tilde{y}$ é sempre solução de:

$$
\tilde{y}^{(1)}(t)=\frac{1}{t^{3}}\left[6\left(\int_{0}^{t} \int_{0}^{\sigma_{1}} \tilde{y}\left(\sigma_{2}\right) d \sigma_{2} \sigma_{1}\right)-18\left(\int_{0}^{t} \sigma_{1} \tilde{y}\left(\sigma_{1}\right) d \sigma_{1}+6 t^{2} \tilde{y}(t)\right] .\right.
$$

Defina:

$$
\Delta(t)=\frac{1}{t^{3}}\left[6\left(\int_{0}^{t} \int_{0}^{\sigma_{1}} R_{3}\left(\sigma_{2}\right) d \sigma_{2} \sigma_{1}\right)-18\left(\int_{0}^{t} \sigma_{1} R_{3}\left(\sigma_{1}\right) d \sigma_{1}+6 t^{2} R_{3}(t)\right]\right.
$$

Então,(2.29) fornece

$$
\widehat{\dot{y}}(t)=\tilde{y}^{(1)}(t)+\Delta(t)
$$

O erro cometido é 


$$
\begin{aligned}
e(t) & =\widehat{\dot{y}}(t)-y^{(1)}(t) \\
& =\tilde{y}^{(1)}(t)+\Delta(t)-\tilde{y}^{(1)}(t)-R_{3}^{(1)}(t) \\
& =\Delta(t)-R_{3}^{(1)}(t)
\end{aligned}
$$

Logo, calculamos um limite superior para $\Delta(t)$ dado por

$$
\begin{aligned}
|\Delta(t)| & =\mid \frac{1}{t^{3}}\left[6\left(\int_{0}^{t} \int_{0}^{\sigma_{1}} R_{3}\left(\sigma_{2}\right) d \sigma_{2} \sigma_{1}\right)-18\left(\int_{0}^{t} \sigma_{1} R_{3}\left(\sigma_{1}\right) d \sigma_{1}+6 t^{2} R_{3}(t)\right] \mid\right. \\
& \leq \frac{1}{\left|t^{3}\right|}\left\{\left|6\left(\int_{0}^{t} \int_{0}^{\sigma_{1}} R_{3}\left(\sigma_{2}\right) d \sigma_{2} \sigma_{1}\right)\right|-\mid 18\left(\int_{0}^{t} \sigma_{1} R_{3}\left(\sigma_{1}\right) d \sigma_{1}|+| 6 t^{2} R_{3}(t) \mid\right\}\right. \\
& \leq \frac{33}{20}|t|^{2} \mathcal{N}
\end{aligned}
$$

Considerando as equações (2.35), (2.36) e (2.34) obtemos

$$
\sup _{\tau \in[0, t]}|e(t)| \leq \frac{33}{20}|t|^{2} \mathcal{N}+\frac{1}{2}|t|^{2} \mathcal{N}
$$

Assim, um limite superior para o erro da estimativa dentro do intervalo $[0, t]$ será:

$$
\sup _{\tau \in[0, t]}|e(t)| \leq \frac{43}{20}|t| \sup _{\tau \in[0, t]}\left|y^{(3)}(t)\right|
$$

Em particular, um limite para $h$ é:

$$
\sup _{\tau \in[0, h]}|e(t)| \leq \frac{43}{20}|h| \sup _{\tau \in[0, h]}\left|y^{(3)}(t)\right|
$$


Logo, derivando (2.32) duas vezes em ambos os lados, obtém-se

$$
y^{(2)}(t)=\tilde{y}^{(2)}(t)+R_{3}^{(2)}(t)
$$

Sabendo que $\tilde{y}^{(2)}(t)=y^{(2)}(0)$, pelo teorema do Valor Médio, existe $\xi \in[0, t]$ tal que $y^{(2)}(t)-y^{(2)}(0)=y^{(3)}(\xi)(t-0)$. Portanto

$$
\left|R_{3}^{(2)}(t)\right| \leq \mathcal{N}|t|
$$

Substituindo $y=\tilde{y}+R_{3}$ na equação (2.30), obtemos:

$$
\begin{aligned}
\widehat{\ddot{y}}(t)= & \frac{1}{t^{3}}\left[6\left(\int_{0}^{t} \tilde{y}\left(\sigma_{1}\right) d \sigma_{1}\right)+3 t^{2} \dot{\tilde{y}}(t)-6 t \tilde{y}(t)\right]+ \\
& \frac{1}{t^{3}}\left[6\left(\int_{0}^{t} \tilde{R}_{3}\left(\sigma_{1}\right) d \sigma_{1}\right)+3 t^{2} R_{3}^{(1)}(t)-6 t R_{3}(t)\right]
\end{aligned}
$$

Lembremos que $\tilde{y}$ é sempre solução de

$$
\tilde{y}^{(2)}(t)=\frac{1}{t^{3}}\left[6\left(\int_{0}^{t} \tilde{y}\left(\sigma_{1}\right) d \sigma_{1}\right)+3 t^{2} \dot{\tilde{y}}(t)-6 t \tilde{y}(t)\right]
$$

Defina:

$$
\Delta(t)=\frac{1}{t^{3}}\left[6\left(\int_{0}^{t} \tilde{R}_{3}\left(\sigma_{1}\right) d \sigma_{1}\right)+3 t^{2} R_{3}^{(1)}(t)-6 t R_{3}(t)\right]
$$

Então, (2.30) fornece:

$$
\hat{\ddot{y}}(t)=\tilde{y}^{(2)}+\Delta(t)
$$

O erro de estimação é: 


$$
\begin{gathered}
e(t)=\hat{\ddot{y}}(t)-y^{(2)} \\
e(t)=\tilde{y}^{(2)}(t)+\Delta(t)-\left(\tilde{y}^{(2)}(t)+R_{3}^{(2)}(t)\right) \\
e(t)=\Delta(t)-R_{3}^{(2)}(t)
\end{gathered}
$$

Logo calculamos um limite superior para $\Delta(t)$

$$
\begin{gathered}
|\Delta(t)|=\left|\frac{1}{t^{3}}\left[6\left(\int_{0}^{t} R_{3}\left(\sigma_{1}\right) d \sigma_{1}\right)+3 t^{2} R_{3}^{(1)}(t)-6 t R_{3}(t)\right]\right| \\
|\Delta(t)| \leq \frac{1}{|t|^{3}}\left|\left(6 \int_{0}^{t} R_{3}\left(\sigma_{1}\right) d \sigma_{1}\right)\right|+\left|3 t^{2} R_{3}^{(1)}(t)\right|+\left|6 t R_{3}(t)\right| \\
|\Delta(t)| \leq \frac{1}{|t|^{3}}\left[\frac{\mathcal{N}|t|^{4}}{4}+\frac{3 \mathcal{N}|t|^{4}}{2}+\mathcal{N}|t|^{4}\right] \\
|\Delta(t)| \leq \frac{1}{|t|^{3}}\left[\frac{11 \mathcal{N}|t|^{4}}{4}\right]
\end{gathered}
$$

Sabendo que: $\left|R_{3}^{(2)}\right| \leq \mathcal{N}|t|$ e que $e(t)=\Delta(t)-R_{3}^{(2)}(t)$

teremos:

$$
|e(t)| \leq \frac{11}{4} \mathcal{N}|t|+\mathcal{N}|t|
$$

Um limite superior para o erro da estimativa dentro do intervalo $[0, t]$ será:

$$
\sup _{\tau \in[0, t]}|e(t)| \leq \frac{15}{4}|t| \sup _{\tau \in[0, t]}\left|y^{(3)}(t)\right|
$$

em particular: 


$$
\sup _{\tau \in[0, h]}|e(t)| \leq \frac{15}{4}|h| \sup _{\tau \in[0, h]}\left|y^{(3)}(t)\right|
$$

\subsubsection{Erro de estimação}

Notemos que existe um tipo de erro que se comete ao realizar a implementação. Como sabemos, nós temos a equação (2.30) a qual será novamente apresentada para facilitar a compreensão do desemvolvimento:

$$
\widehat{\ddot{y}}_{1}(t)=\frac{1}{t^{3}}\left[6\left(\int_{0}^{t} y(\sigma) d \sigma\right)+3 t^{2} \dot{y}(t)+6 t y(t)\right]
$$

Mas na realidade nós desconhecemos o valor exato de $\dot{y}(t)$, então para a implementação consideraremos o valor obtido na estimação da primeira derivada $\hat{\dot{y}}(t)$ no lugar de $\dot{y}(t)$. Assim vamos obter uma equação dada da seguinte forma:

$$
\widehat{\ddot{y}}(t)=\frac{1}{t^{3}}\left[6\left(\int_{0}^{t} y(\sigma) d \sigma\right)+3 t^{2} \hat{\dot{y}}(t)+6 t y(t)\right]
$$

Logo, subtraindo as duas equações anteriores obtemos:

$$
\left\|\hat{\ddot{y}}(t)-\hat{\ddot{y}}_{1}(t)\right\| \leq\left\|\frac{3}{t}(\hat{\dot{y}}(t)-\dot{y}(t))\right\|
$$

Lembrando que das equações (2.41) e (2.38) temos:

$$
\left\|\hat{\ddot{y}}_{1}(t)-\ddot{y}(t)\right\| \leq \frac{15}{4}|t| \sup _{t \in[0, t]}\left|y^{(3)}(t)\right|
$$


$\mathrm{e}$

$$
\|\hat{\dot{y}}(t)-\dot{y}(t)\| \leq \frac{43}{20}|t|^{2} \sup _{t \in[0, t]}\left|y^{(3)}(t)\right|
$$

Substituindo (2.46) em (2.44), obtemos:

$$
\left\|\hat{\ddot{y}}(t)-\hat{\ddot{y}}_{1}(t)\right\| \leq \frac{129}{20}|t| \sup _{t \in[0, t]}\left|y^{(3)}(t)\right|
$$

Depois

$$
\begin{aligned}
\|\ddot{y}(t)-\hat{\ddot{y}}(t)\| & =\left\|\ddot{y}(t)-\hat{\ddot{y}}_{1}(t)+\hat{\ddot{y}}_{1}(t)-\hat{\ddot{y}}(t)\right\| \\
& \leq\left\|\ddot{y}(t)-\hat{\ddot{y}}_{1}(t)\right\|+\left\|\hat{\ddot{y}}_{1}(t)-\hat{\ddot{y}}(t)\right\|
\end{aligned}
$$

Utilizando (2.45) e (2.47), obtemos um erro de estimação final que consideraremos na demonstração dos teoremas:

$$
\|\ddot{y}(t)-\hat{\ddot{y}}(t)\| \leq \frac{51}{5}|t| \sup _{\tau \in[0, t]}\left|y^{(3)}(t)\right|
$$




\section{Capítulo 3}

\section{ESTABILIDADE EM MALHA}

\section{FECHADA}

Este capítulo introduz um teorema que possui natureza semelhante a de um teorema da separação ao realizar o estudo da estabilidade em malha fechada de uma classe de sistemas. Nosso resultado mostrará que mesmo que a dinâmica do sistema em malha fechada seja aumentada pela presença da dinâmica do estimador, a prova da estabilidade em malha fechada somente considera a dinâmica do sistema original alterada pela presença do erro de estimação (2.38) e (2.41).Podese dizer então que o sistema em malha fechada estudado na prova do teorema de estabilidade tem a mesma ordem do sistema em malha aberta, fato que não acontece na maioria das demonstrações do teorema da separação (vide por exemplo (Atassi e Khalil 1999), (Atassi e Khalil 2000) e (Atassi e Khalil 2001)). Na seção 3.2 começaremos introduzindo os resultados da estabilidade exponencial e assintótica para os filtros de segunda ordem (as demonstrações podem ser vistas em (Murgueytio, Silva e Novaes 2013)). Nas seções seguintes faremos um estudo da estabilidade exponencial e assintótica para os filtros de terceira ordem. Embora 
a classe de sistemas seja restrita, acreditamos que as técnicas aqui desenvolvidas podem ser estendidas para classes de sistemas mais gerais.

\subsection{Estabilidade em malha fechada para o filtro de segunda ordem}

Dados $y(t) \in \mathbb{R}^{m}$ e $u(t) \in \mathbb{R}^{l}$. Considere um sistema afim de classe $C^{1}$ com entrada $u(t)$ e saída $y(t)$, da forma:

$$
\begin{aligned}
\dot{y}(t) & =y^{(1)}(t) \\
\dot{y}^{(1)}(t) & =F\left(t, y(t), y^{(1)}(t)\right)+G\left(t, y(t), y^{(1)}(t)\right) u(t)
\end{aligned}
$$

Seja

$$
\begin{aligned}
x(t) & =\left(y(t)^{\top}, y^{(1)}(t)^{\top}\right) \\
f(t, x(t)) & =\left(y^{(1)}(t)^{\top}, F(t, x(t))^{\top}\right)^{\top} \\
g(t, x(t)) & =\left(0, G(t, x(t))^{\top}\right)^{\top} \\
h(t, x(t)) & =y(t) .
\end{aligned}
$$

Logo teremos: 


$$
\begin{aligned}
\dot{x}(t) & =f(t, x(t))+g(t, x(t)) u(t) \\
y(t) & =h(t, x(t)) \\
x\left(t_{0}\right) & =x_{0}
\end{aligned}
$$

Assumiremos que a seguinte realimentação de estado estabiliza assintoticamente a origem do sistema (3.1)

$$
u(t)=\alpha\left(t, y(t), y^{(1)}(t)\right)
$$

Considere-se a seguinte lei de controle:

$$
u(t)=\alpha(t, y(t), \hat{\dot{y}}(t))
$$

Enunciaremos os teoremas 1 e 2 de estabilidade em malha fechada para o estimador de ordem 2. Nota-se que as hipóteses e demonstrações destes teoremas 1 e 2 serão muito semelhantes ao caso de estimadores de terceira ordem que serão vistos na seção seguinte.

HIPÓTESE hip 1. As seguintes hipóteses valem para o sistema (3.1) e para uma lei de controle $(3.5)$ de classe $C^{1}$

- $F(t, 0,0)=0, \forall t \geq t_{0}$.

- Existe $\gamma$ positivo tal que $\left\|G\left(t, y, y^{(1)}\right)\right\| \leq \gamma$, para todos os $y, y^{(1)}$ pertencentes a $\mathbb{R}^{m}$. 
- Seja $x$ definido por (3.2) e seja

$$
P(t, x)=F(t, x)+G(t, x) \alpha(t, x)
$$

Assuma que $\frac{\partial P}{\partial x}$ é uniformemente limitada. Em particular, como $P(t, 0)=$ 0 para todo $t$, existe $\delta>0$ tal que $\|P(t, x)\| \leq \delta\|x\|$.

- $\alpha(t, 0,0)=0, \forall t \geq t_{0}$.

- A aplicação $\alpha$ é (globalmente) Lipschitz com relação à terceira variável, isto é, existe $K>0$ tal que

$$
\begin{aligned}
& \left\|\alpha\left(t, y, y^{(1)}\right)-\alpha\left(t, y, \hat{y}^{(1)}\right)\right\| \leq K\left\|y^{(1)}-\hat{y}^{(1)}\right\|, \\
& \forall y, y^{(1)}, \hat{y}^{(1)} \in \mathbb{R}^{l} \mathrm{e} \forall t \geq t_{0}
\end{aligned}
$$

HIPÓTESE hip 2. A origem do sistema em malha fechada com a realimentação (3.4) é globalmente exponencialmente estável ${ }^{1}$.

Teorema 1. Assuma que aplicamos ao sistema (3.1) a lei de controle (3.5) onde $\alpha(\cdot)$ obedece às hipóteses h1 e h2, e $\hat{\dot{y}}(t)$ é saída do estimador algébrico (2.12) reinicializado periodicamente a cada $h$ segundos. Então existe $\bar{h}$ suficientemente pequeno tal que a origem do sistema (3.1) em malha fechada com a lei de controle (3.5) é globalmente exponencialmente estável, para todo $h \leq \bar{h}$.

HIPÓTESE hip 3. Considere $x(t), f(t, x), g(t, x)$, e $h(t, x)$ definidos como em (3.2). Seja $\alpha$ a lei de controle (3.4). Considere o sistema (3.1) em malha fechada

\footnotetext{
${ }^{1}$ Consulte a Definição 3.5 de (Khalil 1996) para a definição de estabilidade exponencial global.
} 
com (3.4) reescrito como

$$
\begin{aligned}
\dot{x}(t) & =f(t, x(t))+g(t, x(t)) \alpha(t, x(t)) \\
y(t) & =h(x(t)) \\
x\left(t_{0}\right) & =x_{0}
\end{aligned}
$$

Seja $n=2 m$ e assuma que exista uma função $V:\left[t_{0}, \infty\right) \times \mathbb{R}^{n} \rightarrow \mathbb{R}$ de classe $C^{1}$ e funções $W_{i}: \mathbb{R}^{n} \mapsto \mathbb{R}, i=1,2,3$ tais que:

(i) $W_{i}$ é contínua e definida positiva em torno da origem, $i=1,2,3$, sendo que $W_{1}$ é radialmente ilimitada.

(ii) $W_{1}(x) \leq V(t, x) \leq W_{2}(x), \forall t \geq t_{0}$ e $x \in \mathbb{R}^{n}$.

(iii) $\dot{V}(t, x)=\frac{\partial V}{\partial t}+\frac{\partial V}{\partial x} \theta(t, x) \leq-W_{3}$, onde $\theta=f+g \alpha$.

(iv) Existe $\epsilon>0$ suficientemente pequeno tal que a função $W_{4}(x)$ definida por

$$
W_{4}(x)=W_{3}(x)-\epsilon\left\|\frac{\partial V}{\partial x}\right\|\|x\|
$$

é definida positiva em torno da origem para $x \in \mathbb{R}^{n}$.

Teorema 2. Assuma que aplicamos no sistema (3.1) a lei de controle (3.5). Assuma que $\alpha(\cdot)$ obedece às hipóteses $h 1$ e h3. Então existe $\bar{h}$ suficientemente pequeno tal que a origem do sistema (3.1) em malha fechada com a lei de controle (3.5) é globalmente uniformemente assintoticamente estável, para todo $h \leq \bar{h}$.

A prova dos teoremas 1 e 2 pode ser encontrada em (Murgueytio, Silva e Novaes 2013) 


\subsection{Estabilidade em malha fechada para o filtro de terceira ordem}

Seja $y(t) \in \mathbb{R}^{m}$ e $u(t) \in \mathbb{R}^{l}$. Considere um sistema afim de classe $C^{2}$ com entrada $u(t)$ e saída $y(t)$, da forma:

$$
\begin{aligned}
\dot{y}(t) & =y^{(1)}(t) \\
\dot{y}^{(1)}(t) & =y^{(2)}(t) \\
\dot{y}^{(2)}(t) & =F\left(t, y(t), y^{(1)}(t), y^{(2)}(t)\right)+G\left(t, y(t), y^{(1)}(t), y^{(2)}(t)\right) u(t)
\end{aligned}
$$

Seja

$$
\begin{aligned}
x(t) & =\left(y(t)^{\top}, y^{(1)}(t)^{\top}, y^{(2)}(t)^{\top}\right) \\
f(t, x(t)) & =\left(y^{(1)}(t)^{\top}, y^{(2)}(t)^{\top}, F(t, x(t))^{\top}\right)^{\top} \\
g(t, x(t)) & =\left(0,0, G(t, x(t))^{\top}\right)^{\top} \\
h(t, x(t)) & =y(t) .
\end{aligned}
$$

Nota-se que a classe de sistemas estudada é uma subclasse dos sistemas afins variantes no tempo dados por

$$
\begin{aligned}
\dot{x}(t) & =f(t, x(t))+g(t, x(t)) u(t) \\
y(t) & =h(t, x(t)) \\
x\left(t_{0}\right) & =x_{0}
\end{aligned}
$$


Assuma que a origem é um ponto de equilíbrio para o sistema (3.8) para entrada nula. Em outras palavras para o sistema reescrito na forma (3.10) temos

$$
f(t, 0)=0, \forall t \geq t_{0}
$$

Como é usual em teoria de sistemas não lineares, não há perda de generalidade em estudarmos somente a estabilidade da origem. A estabilidade em torno de outros pontos de equilíbrio com $y \neq 0$ é completamente análoga. Por outro lado, para classe de sistemas estudada, $\dot{x}=0$ implica em $y^{(1)}=0$

Assumiremos que uma realimentação de estado

$$
u(t)=\alpha\left(t, y(t), y^{(1)}(t), y^{(2)}(t)\right)
$$

estabiliza assintoticamente a origem do sistema (3.8). Em alguns momentos será mais conveniente utilizar a notação compacta da equação (3.10). Neste caso, sendo coerente com a notação da equação (3.9a), vamos utilizar a notação

$$
\alpha(t, x) \doteq \alpha\left(t, y, y^{(1)}, y^{(2)}(t)\right)
$$

onde $\doteq$ denota "igual por definição". Nos Teoremas 3 e 4, assumimos que a realimentação do estado estimado $\hat{x}$ definido por

$$
\hat{x}=(y, \hat{\dot{y}}, \hat{\ddot{y}}(t))
$$

é aplicada no sistema (3.8). Em outras palavras, a seguinte lei de controle será considerada:

$$
u(t)=\alpha(t, y(t), \hat{\dot{y}}(t), \hat{y}(t))
$$


onde $\hat{\dot{y}}(t)$ e $\hat{\ddot{y}}(t)$ é saída do estimador algébrico (2.22) e (2.23) reinicializado periodicamente a cada $h$ segundos. Em alguns momentos será conveniente denotar esta lei de controle na forma compacta abaixo:

$$
u=\alpha(t, \hat{x})
$$

\subsubsection{Estabilidade exponencial para o filtro de terceira ordem}

Antes de enunciar o primeiro teorema de estabilidade, vamos apresentar as hipóteses adotadas neste teorema.

Assuma que

HIPÓTESE Hip 1. As seguintes hipóteses valem para o sistema (3.8) e para uma lei de controle (3.14) de classe $C^{1}$

- $F(t, 0,0,0)=0, \forall t \geq t_{0}$.

- Existe $\gamma$ positivo tal $\left\|G\left(t, y, y^{(1)}, y^{(2)}\right)\right\| \leq \gamma$, para todos os $y, y^{(1)}, y^{(2)}$ pertencentes a $\mathbb{R}^{m}$.

- Seja $x$ definido por (3.9a) e seja

$$
P(t, x)=F(t, x)+G(t, x) \alpha(t, x)
$$

Assuma que $\frac{\partial P}{\partial x}$ é uniformemente limitada. Em particular, como $P(t, 0)=0$ para todo $t$, existe $^{2} \delta>0$ tal que $\|P(t, x)\| \leq \delta\|x\|$.

- $\alpha(t, 0,0,0)=0, \forall t \geq t_{0}$.

\footnotetext{
${ }^{2}$ A existência de $\delta$ é garantida pela desigualdade do valor médio (Lima 1981).
} 
- A aplicação $\alpha$ é (globalmente) Lipschitz com relação à terceira variável, isto é, existe $K>0$ tal que

$$
\begin{gathered}
\left\|\alpha\left(t, y, y^{(1)}, y^{(2)}\right)-\alpha\left(t, y, \hat{y}^{(1)}, \hat{y}^{(2)}\right)\right\| \leq \mathcal{K}\left\|\left(y^{(1)}, y^{(2)}\right)-\left(\hat{y}^{(1)}, \hat{y}^{(2)}\right)\right\|, \\
\forall y, y^{(1)}, \hat{y}^{(1)} \in \mathbb{R}^{l} \mathrm{e} \forall t \geq t_{0}
\end{gathered}
$$

HIPÓTESE Hip 2. A origem do sistema em malha fechada com a realimentação (3.11) é globalmente exponencialmente estável ${ }^{3}$.

O teorema a seguir apresenta a primeira versão do princípio da separação para esta classe de sistemas aliada aos estimadores algébricos da seção 2.3.

Teorema 3. Assuma que aplicamos ao sistema (3.8) a lei de controle (3.14) onde $\alpha(\cdot)$ obedece às hipótese Hip 1 e Hip 2, $\hat{\dot{y}}(t)$ e $\hat{\ddot{y}}(t)$ é saída do estimador algébrico (2.22) e (2.23) reinicializado periodicamente a cada $h$ segundos. Então existe $\bar{h}$ suficientemente pequeno tal que a origem do sistema (3.8) em malha fechada com a lei de controle (3.14) é globalmente exponencialmente estável para todo $h \leq \bar{h}$

\subsubsection{Estabilidade assintótica para o filtro de terceira ordem}

Apresentamos agora a hipótese do segundo teorema de estabilidade que substitui a hipótese Hip 2.

Observação : A hipótese Hip 3 é bastante natural a menos de sua parte (iv) que é uma hipótese técnica. De fato, isto é confirmado pelo Teorema 6 que é um

\footnotetext{
${ }^{3}$ Consulte a Definição 3.5 de (Khalil 1996) para a definição de estabilidade exponencial global.
} 
teorema converso que será apresentado na prova do Teorema 4.

HIPÓTESE Hip 3. Considere $x(t), f(t, x), g(t, x)$, e $h(t, x)$ definidos como em (3.9). Seja $\alpha$ a lei de controle (3.11). Considere o sistema (3.8) em malha fechada com (3.11) reescrito como

$$
\begin{aligned}
\dot{x}(t) & =f(t, x(t))+g(t, x(t)) \alpha(t, x(t)) \\
y(t) & =h(x(t)) \\
x\left(t_{0}\right) & =x_{0}
\end{aligned}
$$

Seja $n=2 m$ e assuma que existe uma função $V:\left[t_{0}, \infty\right] \times \mathbb{R}^{n} \rightarrow \mathbb{R}$ de classe $C^{1}$ e funções $W_{i}: \mathbb{R}^{n} \mapsto \mathbb{R}, i=1,2,3$ tais que:

(i) $W_{i}$ é contínua e definida positiva em torno da origem, $i=1,2,3$, sendo que $W_{1}$ é radialmente ilimitada.

(ii) $W_{1}(x) \leq V(t, x) \leq W_{2}(x), \forall t \geq t_{0}$ e $x \in \mathbb{R}^{n}$.

(iii) $\dot{V}(t, x)=\frac{\partial V}{\partial t}+\frac{\partial V}{\partial x} \theta(t, x) \leq-W_{3}$, onde $\theta=f+g \alpha$.

(iv) Existe $\epsilon>0$ suficientemente pequeno tal que a função $W_{4}(x)$ definida por

$$
W_{4}(x)=W_{3}(x)-\epsilon\left\|\frac{\partial V}{\partial x}\right\|\|x\|
$$

é definida positiva em torno da origem para $x \in \mathbb{R}^{n}$.

Teorema 4. Assuma que aplicamos no sistema (3.8) a lei de controle (3.14). Assuma que $\alpha(\cdot)$ obedece às hipóteses Hip 1 e Hip 3. Então existe $\bar{h}$ suficientemente pequeno tal que a origem do sistema (3.8) em malha fechada com a lei de controle (3.14) é globalmente uniformemente assintoticamente estável, para todo $h \leq \bar{h}$ 


\subsection{Prova dos Teoremas}

\subsubsection{Considerações comuns aos teoremas 3 e 4}

Note que o sistema em malha fechada com a realimentação de estado estimado é da forma

$$
\begin{aligned}
\dot{y}(t) & =y^{(1)}(t) \\
\dot{y}^{(1)}(t) & =y^{(2)}(t) \\
\dot{y}^{(2)}(t) & =F\left(t, y(t), y^{(1)}(t), y^{(2)}\right) \\
& +G\left(t, y(t), y^{(1)}(t), y^{(2)}\right) \alpha(t, y(t), \hat{\dot{y}}(t), \hat{\ddot{y}}(t))
\end{aligned}
$$

onde $\hat{\dot{y}}$ e $\hat{y}$ são obtidos de (2.22) e (2.23). A demostração de estabilidade considerará $\hat{\dot{y}}$ e $\hat{\ddot{y}}$ como uma função do tempo, ou seja, a ordem do sistema em malha fechada será a ordem do sistema original.

A prova considera o sistema em malha fechada com estimador (3.19) como uma aproximação do sistema em malha fechada com uma realimentação estabilizante (3.18) expresso assim:

$$
\begin{aligned}
\dot{y}(t) & =y^{(1)}(t) \\
\dot{y}^{(1)}(t) & =y^{(2)}(t) \\
\dot{y}^{(2)}(t) & =F\left(t, y(t), y^{(1)}(t), y^{(2)}\right) \\
& +G\left(t, y(t), y^{(1)}(t), y^{(2)}\right) \alpha(t, y(t), \dot{y}(t), \ddot{y}(t))
\end{aligned}
$$


Em outras palavras, o sistema (3.19) é o sistema (3.20) alterado por uma incerteza, que é limitada pelo erro de estimação. Sendo assim, prova-se que o estado do sistema (3.19) é convergente para o ponto de equilíbrio através da mesma função de Lyapunov do sistema (3.20), que continua definida negativa mesmo na presença do erro de estimação

De (3.19c), (2.48) e (2.39) teremos:

$$
\begin{aligned}
\|e(t)\| & =\|\left(y^{(1)}(t)-\hat{\dot{y}}(t), y^{(2)}(t)-\hat{\ddot{y}}(t) \|\right. \\
& \leq h L\|F(t, x)+G(t, x) \alpha(t, y, \hat{\dot{y}}, \hat{y})\|
\end{aligned}
$$

onde $L=\frac{43}{20} h+\frac{51}{5}$. Da desigualdade triangular segue-se que

$$
\begin{aligned}
\|e(t)\| \leq & h L\left\|F+G \alpha\left(t, y, y^{(1)}, y^{(2)}\right)\right\|+ \\
& h L\left\|G\left[\alpha(t, y, \hat{\dot{y}}, \hat{\ddot{y}})-\alpha\left(t, y, y^{(1)}, y^{(2)}\right)\right]\right\|
\end{aligned}
$$

Agora usamos a aplicação $P(t, x)$ definida na hipóteses Hip 1, a propriedade de Lipschitz de $\alpha$, e o conhecimento de que $G$ é uniformemente ilimitada, isto é $G \leq \delta$

$$
\begin{aligned}
\|e(t)\| & \leq h L\|P(t, x)\|+h L \gamma \mathcal{K}\|e(t)\| \\
& \leq h L \delta\|x\|+h L \gamma \mathcal{K}\|e(t)\|
\end{aligned}
$$


Assim:

$$
\|e(t)\| \leq \frac{h L \delta\|x\|}{1-h L \gamma \mathcal{K}}
$$

Logo, substituindo L:

$$
\|e(t)\| \leq \frac{h\left(\frac{43}{20} h+\frac{51}{5}\right) \delta\|x\|}{1-h\left(\frac{43}{20} h+\frac{51}{5}\right) \delta \mathcal{K}}
$$

Se $h$ for suficientemente pequeno de modo que $h\left(\frac{43}{20} h+\frac{15}{4}\right) \delta \mathcal{K} \leq 1$, então

$$
\|e(t)\| \leq h \mu\|x\|
$$

onde $\mu=h L \delta /(1-h L \gamma \mathcal{K})>0$.

Usando as notações (3.9), (3.12), (3.13) e (3.15), escrevemos (3.18) na forma

$$
\begin{aligned}
\dot{x} & =f(t, x)+g(t, x)[\alpha(t, \hat{x})] \\
& =f(t, x)+g(t, x) \alpha(t, x)+g[\alpha(t, \hat{x})-\alpha(t, x)]
\end{aligned}
$$

logo denotando:

$$
\theta(t, x)=f(t, x)+g(t, x) \alpha(t, x)
$$

Escrevemos o sistema em malha fechada 


$$
\dot{x}(t)=\theta(t, x(t))+g[\alpha(t, \hat{x})-\alpha(t, x)]
$$

onde $\hat{x}$ é definido por (3.13). Se $V(t, x)$ for uma função de Lyapunov para o sistema, teremos:

$$
\dot{V}=\frac{\partial V}{\partial t}+\frac{\partial V}{\partial x} \theta(t, x)+\frac{\partial V}{\partial x} g[\alpha(t, \hat{x})-\alpha(t, x)]
$$

Seja

$$
\phi=-\left(\frac{\partial V}{\partial t}+\frac{\partial V}{\partial x} \theta(t, x)\right)
$$

Como $\| G \mid \leq \gamma$, de (3.9c) segue-se que $\|g\| \leq \gamma$. Portanto, da propriedade de Lipschitz para $\alpha$ que:

$$
\dot{V} \leq-\phi+\gamma \mathcal{K}\left\|\frac{\partial V}{\partial x}\right\|\|e\|
$$

Podemos deduzir então de (3.21) que

$$
\dot{V} \leq-\phi+h \gamma \mathcal{K} \mu\left\|\frac{\partial V}{\partial x}\right\|\|x\|
$$

\subsubsection{Prova do teorema 3}

Antes de iniciar a demonstração são enunciados dois teoremas de Lyapunov e dois corolários que serão utilizados para as demostrações:

Teorema 5. (Khalil 1996, Teo. 3.8) 
Seja $x=0$ um ponto de equilíbrio para o sistema

$$
\dot{x}(t)=\theta(t, x(t))
$$

$\operatorname{com} \theta:[0, \infty) \times \mathbb{R}^{n} \rightarrow \mathbb{R}$. Se $V:[0, \infty) \times \mathbb{R}^{n} \rightarrow \mathbb{R}$ é uma função continuamente diferenciavel tal que:

- $W_{1}(x) \leq V(t, x) \leq W_{2}(x)$

- $\frac{\partial V}{\partial t}+\frac{\partial V}{\partial x} f(t, x) \leq-W_{3}(x)$

$\forall t \geq 0, \forall x \in D$ onde $W_{1}(x), W_{2}(x)$, e $W_{3}(x)$ são funções continuas definidas positivas sob D. Então, $x=0$ é uniformemente assintoticamente estável.

Teorema 6. (Khalil 1996, Teo. 3.12)

Seja

$$
\dot{x}(t)=\theta(t, x(t))
$$

um sistema, com $\theta: \mathbb{R} \times \mathbb{R}^{n} \rightarrow \mathbb{R}^{n}$ de classe $C^{1}$, possuindo um ponto de equilíbrio na origem que é globalmente exponencialmente estável. Assuma que $\frac{\partial \theta}{\partial x}$ é uniformemente limitado. Então existem constantes positivas $c_{1}, c_{2}, c_{3}$, e $c_{4}$, e uma função de Lyapunov $V: \mathbb{R} \times \mathbb{R}^{n} \rightarrow \mathbb{R}$ de classe $C^{1}$ tal que

- $c_{1}\|x\|^{2} \leq V(t, x) \leq c_{2}\|x\|^{2}$.

- $\dot{V}=\frac{\partial V}{\partial t}+\frac{\partial V}{\partial x} \theta(t, x) \leq-c_{3}\|x\|^{2}$.

- $\left\|\frac{\partial V}{\partial x}\right\| \leq c_{4}\|x\|$.

Corolário 1. (Khalil 1996, Cor. 3.3) Suponha que todas as hipóteses do teorema 5 são satisfeitas globalmente (para todo $x \in \mathbb{R}^{n}$ ) e $W_{1}(x)$ é radialmete limitada. Então, $x=0$ é globalmente uniformemente assintoticamente estável. 
Corolário 2. (Khalil 1996, Cor. 3.4) Suponha que todas as hipóteses do teorema 5 são satisfeitas com

$$
W_{1}(x) \geq k_{1}\|x\|^{c}, \quad W_{2}(x) \geq k_{2}\|x\|^{c}, \quad W_{3}(x) \geq k_{3}\|x\|^{c}
$$

para algumas constantes positivas $k_{1}, k_{2}, k_{3}$ e c. Então, $x=0$ é exponencialmente estável. Alem disso, se as hipóteses se mantêm globalmente, então $x=0$ é globalmente exponencialmente estável.

Para iniciar a prova do Teorema 3, note que, se

$$
\theta=\left[\begin{array}{c}
y^{(1)} \\
y^{(2)} \\
P(t, x)
\end{array}\right]
$$

onde P é dado pela equação (3.16) do enunciado da Hipotese Hip 1. Então

$$
\frac{\partial \theta}{\partial x}=\left[\begin{array}{c}
U \\
\frac{\partial P}{\partial x}
\end{array}\right]
$$

onde

$$
U=\left[\begin{array}{ccc}
0 & I_{m} & 0 \\
0 & 0 & I_{m}
\end{array}\right]
$$

Portanto, como $\left\|\frac{\partial P}{\partial x}\right\|$ é uniformemente limitada (vide hipótese Hip 1), teremos que $\frac{\partial \theta}{\partial x}$ é também uniformemente limitada. Utizaremos a função de Lyapunov $V$ cuja existência é garantida pelo Teorema 6. A partir de (3.24) e (3.25), segue-se que

$$
\dot{V} \leq-c_{3}\|x\|^{2}+h \gamma \mathcal{K} \mu c_{4}\|x\|^{2}
$$


assim, para $h$ suficientemente pequeno teremos $N=c_{3}-h \gamma \mathcal{K} \mu c_{4}>0$ e ainda

$$
\dot{V} \leq-N\|x\|^{2}
$$

Esta última condição aliada à primeira e à terceira do teorema converso provam a estabilidade exponencial global do sistema em malha fechada com a realimentação do estado estimado (vide Corolário 2).

\subsubsection{Prova do Teorema 4}

De (3.25) e da parte (iii) da hipótese Hip 3 teremos:

$$
\dot{V} \leq-W_{3}+h \gamma \mathcal{K} \mu\left\|\frac{\partial V}{\partial x}\right\|\|x\|
$$

Agora assuma que $h<\epsilon /(\gamma \mathcal{K} \mu)$, onde $\epsilon$ é definido na parte (iv) de Hip 3. Segue-se que

$$
\dot{V} \leq-W_{4}
$$

Desta última condição, das partes (i) e (ii) da hipótese Hip 3, e do Corolário 1 de (Khalil 1996), segue-se que a origem é globalmente uniformemente assintoticamente estável.

\subsection{Implementação sem reinicialização}

Até agora o método algébrico necessita de uma reinicialização a cada h segundos para garantir a precisão da estimação, mas com o seguinte procedimento não vamos precisar reinicializar estes cálculos. 
Vamos começar com o estimador de segunda ordem. Dada a equação de forma geral (2.11):

$$
\hat{\dot{y}}(t)=\frac{1}{\left(t-t_{0}\right)^{2}}\left[-2 \int_{t_{0}}^{t} y(\sigma) d \sigma+2\left(t-t_{0}\right) y(t)\right]
$$

fazendo $t=t_{0}+h$, obtemos:

$$
\hat{\dot{y}}\left(t_{0}+h\right)=\frac{1}{h^{2}}\left[-2 \int_{t_{0}}^{t_{0}+h} y(\sigma) d \sigma+2 h y\left(t_{0}+h\right)\right]
$$

Logo consideramos $t=t_{0}+h$, obtendo

$$
\hat{\dot{y}}(t)=\frac{1}{h^{2}}\left[-2 \int_{t-h}^{t} y(\sigma) d \sigma+2 h y(t)\right]
$$

Para fazer a implementação de (3.26) devemos efetuar a integração mostrada na equação. A ideia utilizada será o método numérico conhecido como "Regra ou Fórmula do Trapézio" como dado na figura 3.1.

Onde: $r=\frac{h}{n}$ e $t=k r$ 


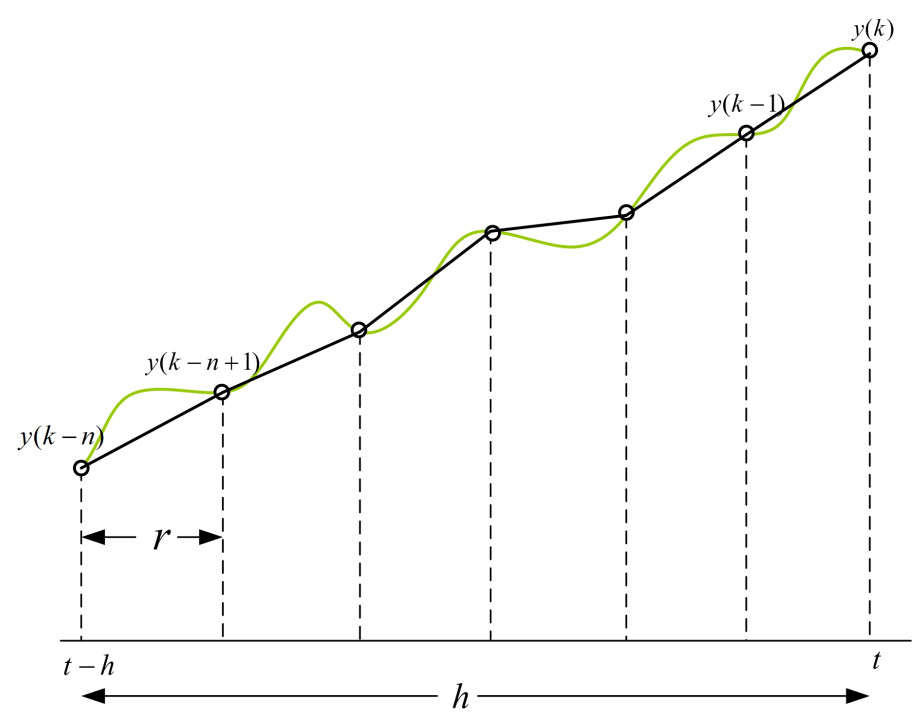

Figura 3.1: Regra do Trapézio

Assim:

$$
\begin{aligned}
\int_{t-h}^{t} y(\sigma) d \sigma \cong & \frac{y(k-n)+y(k-n+1)}{2} r+ \\
& \frac{y(k-n+1)+y(k-n+2)}{2} r+ \\
& \frac{y(k-n+2)+y(k-n+3)}{2} r+ \\
& \ldots \\
& \frac{y(k-2)+y(k-1)}{2} r+ \\
& \frac{y(k-1)+y(k)}{2} r
\end{aligned}
$$

Então:

$$
\int_{t-h}^{t} y(\sigma) d \sigma \cong \frac{y(k)+y(k-n)}{2} r+r \sum_{j=k-n+1}^{k-1} y(j)
$$

Cuja implementação em filtro digital será dada por: 


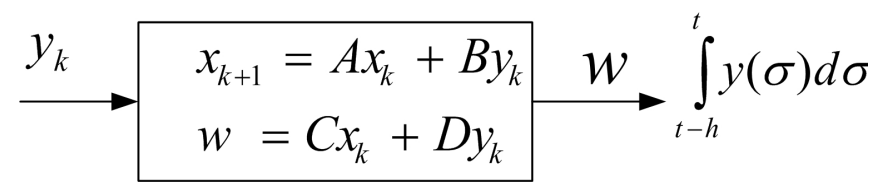

Figura 3.2: Implementação digital da integração pelo método trapezoidal

onde:

$$
\begin{gathered}
A=\left(\begin{array}{ccccc}
0 & 1 & 0 & \ldots & 0 \\
0 & 0 & 1 & \ldots & 0 \\
\vdots & \vdots & \vdots & \ddots & \vdots \\
0 & 0 & 0 & \ldots & 1 \\
0 & 0 & 0 & \ldots & 0
\end{array}\right), \quad B=\left(\begin{array}{c}
0 \\
0 \\
\vdots \\
0 \\
1
\end{array}\right), \quad x_{k}=\left(\begin{array}{c}
y(k-n) \\
y(k-n+1) \\
\vdots \\
y(k-2) \\
y(k-1)
\end{array}\right) \\
C=\left(\begin{array}{ll}
\frac{r}{2} & r \ldots r
\end{array}\right), \quad \text { e } D=\left(\frac{r}{2}\right)
\end{gathered}
$$

Logo mostramos o diagrama de blocos da implementação do estimador na figura 3.3 .

Por outro lado, no caso de querermos implementar o estimador de terceira ordem, da equação (2.23), obteremos a forma geral seguinte:

$$
\widehat{\dot{y}}(t)=\frac{1}{\left(t-t_{0}\right)^{3}}\left[6\left(\int_{t_{0}}^{t} \int_{t_{0}}^{\sigma_{1}} y\left(\sigma_{2}\right) d \sigma_{2} d \sigma_{1}\right)-18\left(\int_{t_{0}}^{t}\left(\sigma_{1}-t_{0}\right) y\left(\sigma_{1}\right) d \sigma_{1}+6\left(t-t_{0}\right)^{2} y(t)\right]\right.
$$

Neste caso teremos uma integral dupla, para resolver: 


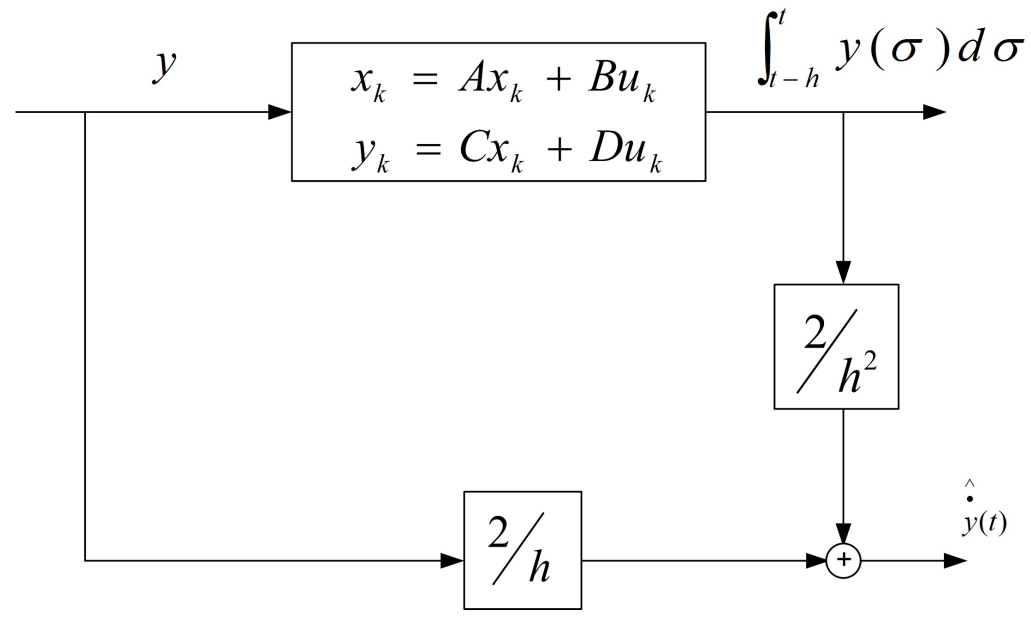

Figura 3.3: Diagrama de Blocos da Integral

$$
\int_{t_{0}}^{t}\left(\int_{t_{0}}^{\sigma_{1}} y\left(\sigma_{2}\right) d \sigma_{2}\right) d \sigma_{1}
$$

Considere-se:

$$
\begin{gathered}
\varphi\left(\sigma_{1}\right)=\int_{t_{0}}^{\sigma_{1}} y\left(\sigma_{2}\right) d \sigma_{2}, \quad \Rightarrow \quad d \varphi=y d \sigma_{1} \\
\psi\left(\sigma_{1}\right)=\sigma_{1}, \quad \Rightarrow \quad d \psi=d \sigma_{1}
\end{gathered}
$$

Utilizando integração por partes, lembrando que: $\psi(t)=t, \quad \varphi\left(t_{0}\right)=0$ 


$$
\begin{aligned}
\int_{t_{0}}^{t} \varphi d \psi & =\left.\varphi \psi\right|_{t} ^{t_{0}}-\int_{t_{0}}^{t} \psi d \varphi \\
& =\varphi(t) \psi(t)-\varphi\left(t_{0}\right) \psi\left(t_{0}\right)-\int_{t_{0}}^{t} \psi d \varphi \\
& =t \int_{t_{0}}^{t} y\left(\sigma_{1}\right) d \sigma_{1}-\int_{t_{0}}^{t} \sigma_{1} y\left(\sigma_{1}\right) d \sigma_{1}
\end{aligned}
$$

Logo, fazemos $t_{0}=t-h$ e obtemos:

$$
\int_{t_{0}}^{t} \varphi d \psi=t \int_{t-h}^{t} y\left(\sigma_{1}\right) d \sigma_{1}-\int_{t-h}^{t} \sigma_{1} y\left(\sigma_{1}\right)=\int_{t-h}^{t}\left(t-\sigma_{1}\right) y\left(\sigma_{1}\right) d \sigma_{1}
$$

Substituindo a equação anterior na equação (3.27), e fazendo $t_{0}=t-h$ e $\sigma_{1}=\sigma$, obtemos:

$$
\widehat{\dot{y}}(t)=\frac{1}{h^{3}}\left[6 \left(\int_{t-h}^{t}(t-\sigma) y(\sigma) d \sigma-18\left(\int_{t-h}^{t}(\sigma-t+h) y(\sigma) d \sigma+6 h^{2} y(t)\right]\right.\right.
$$

Fazendo o mesmo procedimento para a fórmula (2.28), obtemos:

$$
\widehat{\ddot{y}}(t)=\frac{1}{h^{3}}\left[6\left(\int_{t-h}^{t} y(\sigma) d \sigma+3 h^{2} \widehat{\dot{y}}(t)-6 h y(t)\right]\right.
$$

Observação : As deduções dos teoremas 1,2, 3 e 4 podem ser adaptadas facilmente à implementação dos estimadores descrita nesta seção. 


\section{Capítulo 4}

\section{APLICAÇÃO NO CONTROLE DE UM MANCAL}

\section{MAGNETICO}

Neste capítulo aplicaremos os estimadores algébricos para estimação de estado de um sistema de levitação magnética (Moraes e Silva 2015a, Moraes e Silva 2015b). Um sistema de controle por linearização exata será proposto. Para tornar o controle robusto, a derivação de uma das equações foi feita de modo a eliminar o desconhecimento da massa (carga).

Simulações do sistema em malha fechada mostraram uma excelente performance do estimador aliado ao controle proposto. Para simular o sistema utilizaremos dois modelos. Um primeiro modelo rígido e um segundo modelo flexível.

É importante notar que o sistema de controle proposto é projetado a partir do modelo rígido, mas é testado em ambos os modelos com diversas variações para avaliar a robustez. 


\subsection{Modelo do mancal magnético}

A força magnética de um mancal magnético é dada por (4.1) como visto em (Stephan et al. 2013).

$$
F=k_{m} \frac{u^{2}}{x_{1}^{2}}
$$

onde:

$u=$ corrente na bobina em A.

$x_{1}=$ gap em m.

$k_{m}=$ constante de força mancal $N m^{2} / A^{2}$.

\subsubsection{Modelo rígido}

Nesta seção apresentamos o modelo rígido da planta.

Note que:

$x_{1}$ é a posição em metros, $x_{2}=\dot{x}_{1}, k_{d}$ é a constante do sensor em volts por metro, $g$ é a aceleração da gravidade, $M_{r}$ é a massa "real" do corpo levitado e $F$ a força aplicada pelo mancal magnético.

Da figura 4.1 teremos que o sistema descrito no espaço de estados é dado da seguinte forma:

$$
\dot{x}=A x+B\left(-F\left(u, x_{1}\right)+g_{1}\left(x_{1}\right)\right)
$$




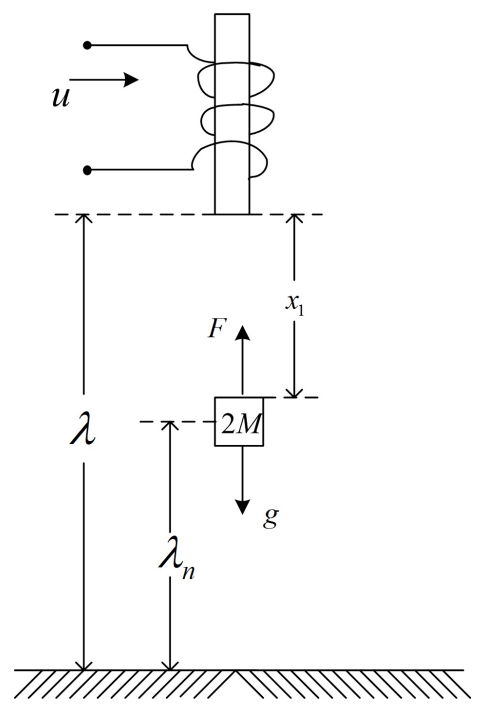

Figura 4.1: Modelo para controle do Mancal Magnético (Modelo Rígido)

onde:

$$
x=\left(\begin{array}{c}
x_{1} \\
x_{2}
\end{array}\right), \quad A=\left(\begin{array}{cc}
0 & 1 \\
0 & 0
\end{array}\right), \quad B=\left(\begin{array}{c}
0 \\
\frac{1}{M_{r}}
\end{array}\right)
$$

Lembrando também que $M_{r}=2 M$ e que $g_{1}\left(x_{1}\right)=\varphi\left(x_{1}\right)$. O termo $g_{1}$ modela a ação da gravidade e o contato da massa com o solo. A razão de incluir um contato elástico é representar algo mais próximo da realidade, já que o contato da massa com o solo não deixa de existir instantaneamente. Mais ainda o modelo com descontinuidade gera problemas numéricos sem interpretação física. A função $g_{1}$ é definida por:

$$
\varphi\left(x_{1}\right)=\left\{\begin{array}{l}
0, \quad x_{1} \geq \lambda \\
-\frac{M_{r} g}{\lambda_{n}}\left(x_{1}-\lambda\right), \quad \lambda-\lambda_{n}<x_{1}<\lambda \\
M_{r} g, \quad x_{1} \leq \lambda-\lambda_{n}
\end{array}\right.
$$


Note que o modelo acima considera $x_{1}=\lambda$ como sendo o contato perfeito no solo e para $x_{1}<\lambda-\lambda_{n}$ temos a ausência de interação entre o solo e a massa. Para $\lambda-\lambda_{n}<x_{1}<\lambda$ temos o anulamento parcial pelo contato (elástico) com o solo. Resaltamos que $\lambda_{n}$ modela a altura a partir do qual há um "contato parcial" com o solo. Isto representa algum tipo de flexibilidade do solo. Para $x_{1}$ entre esses valores a flexibilidade do contato solo/massa é modelada pela função $\varphi$ proposta.

A tabela 1 abaixo mostra os valores que serão usados na simulação do sistema flexível do mancal magnético.

\begin{tabular}{|c|c|}
\hline$g=9,8$ & Aceleração da gravidade $\mathrm{m} / \mathrm{s}^{2}$ \\
\hline$G \phi=1 * 10^{-3}$ & Gap inicial em metros \\
\hline$G=3 * 10^{-4}$ & Gap desejado em metros \\
\hline$K=2 * 10^{5}$ & Constante da mola $\mathrm{N} / \mathrm{m}$ \\
\hline$k_{m}=5 * 10^{-6}$ & Constante de força mancal $N \mathrm{~m}^{2} / \mathrm{A}^{2}$ \\
\hline$k_{d}=2000$ & Constante do sensor de posição em $\mathrm{V} / \mathrm{m}$ \\
\hline$M=2$ & Massa real de cada elemento (flexível) \\
\hline$M_{r}=2 * M$ & Massa real do modelo rígido \\
\hline$\lambda=k d * G \phi$ & Distancia do solo ao mancal \\
\hline$\lambda_{n}=\lambda / 200$ & Altura de "contato parcial " com o solo \\
\hline
\end{tabular}

Tabela 4.1: Valores dos dados utilizados na simulação

\subsubsection{Modelo flexível}

O modelo proposto é da forma :

$$
\dot{x}=A_{f}+B_{f}\left(\begin{array}{c}
-F \\
0
\end{array}\right)+B_{f}\left(\begin{array}{l}
g_{1}\left(x_{1}\right) \\
g_{2}\left(x_{2}\right)
\end{array}\right)
$$

onde: 

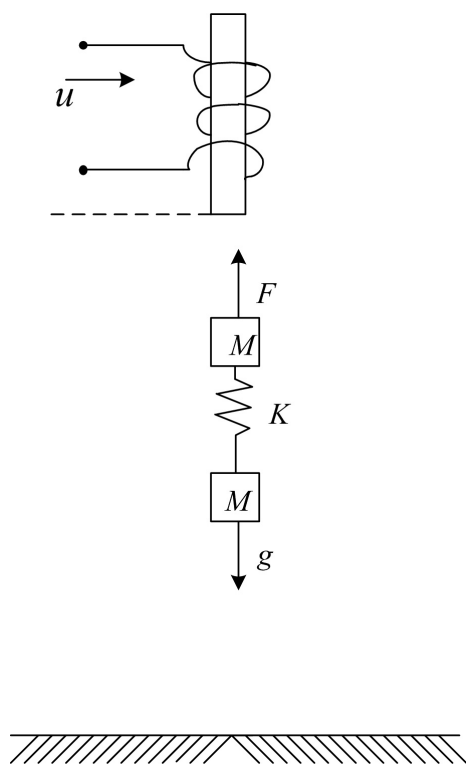

Figura 4.2: Modelo para simulação do mancal magnético (Modelo flexível)

$$
\begin{gathered}
A_{f}=\left(\begin{array}{cc}
0 & I_{2} \\
-M_{1}^{-1} k_{1} & 0
\end{array}\right), \quad B_{f}=\left(\begin{array}{c}
0 \\
M_{1}^{-1}
\end{array}\right) \\
M_{1}=\left(\begin{array}{cc}
M & 0 \\
0 & M
\end{array}\right), \quad k_{1}=\left(\begin{array}{cc}
k & -k \\
-k & k
\end{array}\right)
\end{gathered}
$$

Então:

- O vetor de estados é da forma:

$$
x=\left(\begin{array}{c}
x_{1} \\
x_{2} \\
x_{3} \\
x_{4}
\end{array}\right), \quad x_{3}=\dot{x}_{1}, \quad x_{4}=\dot{x}_{2}
$$

- $F$ é dado pela equação (4.1), $g_{1}\left(x_{1}\right)=\varphi\left(x_{1}\right)$ e $g_{2}\left(x_{2}\right)=\varphi\left(x_{2}\right)$, onde $\varphi$ é dado 
pela equação $(4.2)$

Tal modelo procura captar o contato no solo de um corpo flexível. Note que quando $x_{1}$ é igual a $x_{2}$ não há efeito de mola. Optou-se por modelar o contato no solo agindo nas duas massas, embora poderíamos também assumir $g_{1}\left(x_{1}\right)=0$, que modelaria o contato no solo apenas da massa inferior. Os resultados de simulação obtidos são análogos com essa segunda escolha.

Normaliza-se todas as coordenadas em volts no sensor através da transformação de similaridade descritas na tabela a seguir:

\begin{tabular}{|c|c|}
\hline Rígido & Flexível \\
\hline \hline$\tilde{A}=T^{-1} A T$ & $\tilde{A}_{f}=T^{-1} A_{f} T$ \\
\hline$\tilde{B}=T^{-1} B$ & $\tilde{B}_{f}=T^{-1} B_{f}$ \\
\hline$\tilde{C}=C T$ & $\tilde{C}_{f}=C_{f} T$ \\
\hline$\tilde{D}=D$ & $\tilde{D}_{f}=D_{f}$ \\
\hline$T=\frac{1}{k_{d}} I_{2}$ & $T=\frac{1}{k_{d}} I_{4}$ \\
\hline
\end{tabular}

Tabela 4.2: Modelo rígido e flexível

Denotamos $\tilde{x}_{1}=k_{d} x_{1}$, sendo que $\tilde{x}_{1}$ é a saída do sensor de posição em volts. Note que $\tilde{x}_{2}=\dot{\tilde{x}}_{1}$ para o modelo rígido e para o modelo flexível $\tilde{x}_{2}=\dot{\tilde{x}}_{1}$ e $\tilde{x}_{4}=\dot{\tilde{x}}_{3}$.

\subsection{Linearização exata (LE)}

Considere a trajetória desejada $y_{r e f}(t)$. Defina o erro de rastreamento e suas derivadas.

$$
z_{1}(t)=\tilde{x}_{1}(t)-y_{\text {ref }}(t) \quad(\text { em } \quad \text { volts })
$$




$$
\begin{aligned}
& z_{2}(t)=\tilde{x}_{1}^{(1)}(t)-y_{\text {ref }}^{(1)}(t) \quad(\text { em } \quad \text { volts } / s) \\
& z_{3}(t)=\tilde{x}_{1}^{(2)}(t)-y_{\text {ref }}^{(2)}(t) \quad\left(\text { em } \quad \text { volts } / s^{2}\right)
\end{aligned}
$$

Como sinal de referência $y_{r e f}$ utilizaremos arcos de parábola de modo a termos $y_{r e f}^{(3)}=0$, ver figura 4.3 , com o custo de ter saltos em $y_{r e f}^{(2)}$.

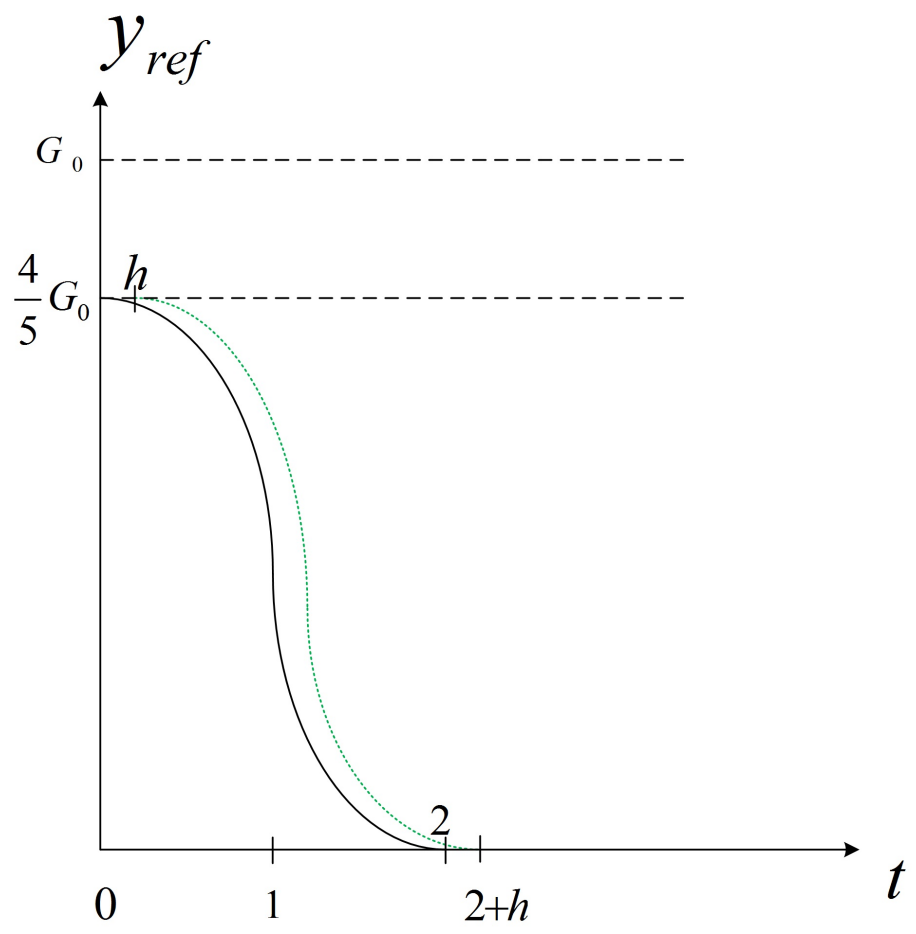

Figura 4.3: Gráfico do sinal de referência $y_{r e f}$

Embora a referência assim construída não possa ser perfeitamente rastreável (pois $y_{\text {ref }}^{(3)}$ não está definida em $t=1$ nem em $t=2$ ), utilizaremos ganhos suficientemente altos para que os saltos da figura 4.4 não provoquem saltos grandes no erro de rastreamento $z_{1}(t)$.

Para dedução da lei de controle por linearização exata consideremos o modelo rígido sem contato com o solo. O contato com o solo será considerado na simulação, 


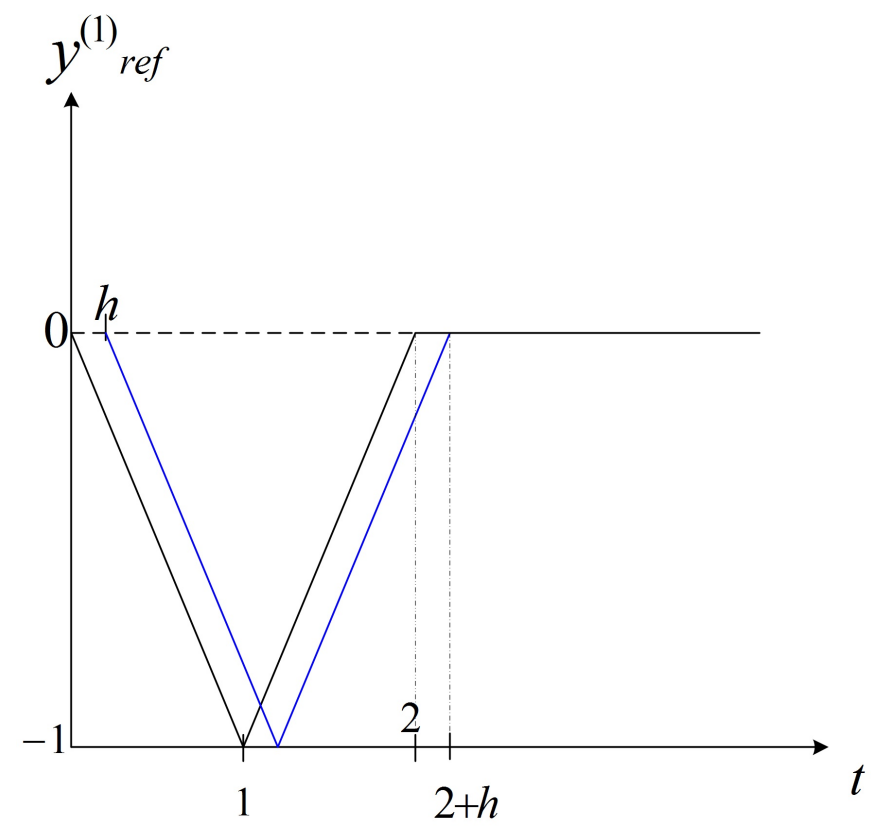

Figura 4.4: Gráfico da derivada do sinal de referência $y_{r e f}^{(1)}$

teremos então:

$\dot{z}_{1}=z_{2}$

$\dot{z}_{2}=\tilde{x}_{1}^{(2)}(t)-y_{\text {ref }}^{(2)}(t)$

Logo:

$\dot{z}_{2}=\frac{k_{d}}{M_{r}}\left[-F\left(\frac{\tilde{x}_{1}}{k_{d}}, u\right)+M_{r} g+f\right]-y_{r e f}^{(2)}$

Onde:

$F=\frac{k_{m} u^{2}}{\left(\frac{\tilde{x}_{1}}{k_{d}}\right)^{2}}, M_{r}=2 M$ e $f$ é uma força externa desconhecida.

Assim teremos que:

$\dot{z}_{2}=\frac{k_{d}}{M_{r}}\left[-F+M_{r} g+f\right]-y_{\text {ref }}^{(2)}$ 
Fazendo $\dot{z}_{2}=v$, sendo $v$ a nova entrada externa, obtemos:

$-F \frac{k_{d}}{M_{r}}=-k_{d} g-\frac{k_{d}}{M_{r}} f+y_{r e f}^{(2)}+v$

Logo:

$$
\frac{k_{m} u^{2}}{\left(\frac{\tilde{x}_{1}}{k_{d}}\right)^{2}} \frac{k_{d}}{M_{r}}=k_{d} g+k_{d} f-y_{r e f}^{(2)}-v
$$

Da equação (4.3), obtemos:

Defina:

$$
\eta=\sqrt{\frac{M_{p}}{k_{d p}}\left(\frac{\tilde{x}_{1}}{k_{d p}}\right)^{2} \frac{1}{k_{m p}}\left[k_{d p} g+\frac{k_{d p}}{M_{p}} f-y_{r e f}^{(2)}-v\right]}
$$

Onde $M_{p}, k_{m p}$ e $k_{d p}$ são os valores presumidos de $M_{r}, k_{m}$ e $k_{d}$ respectivamente.

Considere também a lei de controle linearizante:

$$
u= \begin{cases}\eta & \text { se } \eta \geq 0 \\ 0 & \text { se } \eta<0 \quad \text { (região de saturação) }\end{cases}
$$

A razão da região de saturação da (4.5) é que a força magnética nunca é repulsiva. Prosseguindo com a dedução das equações do sistema em malha fechada teremos:

$\dot{z}_{1}=z_{2}$
$\dot{z}_{2}=\frac{k_{d}}{M_{r}}\left[\frac{-k_{m} u^{2}}{\frac{\tilde{x}_{1}^{2}}{k_{d}^{2}}}+M_{r} g+f\right]-y_{r e f}^{(2)}$

Lembrando que fora da região de saturação $u^{2}=\eta$, e portanto 


$$
\dot{z}_{2}=\frac{-k_{d}^{3} k_{m} M_{p} \tilde{x}_{1}^{2}}{M_{r} \tilde{x}_{1}^{2} k_{d p}^{3} k_{m p}}\left[k_{d p} g+\frac{k_{d p}}{M_{p}} f-y_{r e f}^{(2)}-v\right]+k_{d} g+\frac{k_{d}}{M_{r}} f-y_{r e f}^{(2)}
$$

Fazendo:

$$
K_{1}=\frac{k_{d}^{3} k_{m} M_{p}}{k_{d p}^{3} k_{m p} M_{r}}
$$

Obtemos:

$$
\dot{z}_{2}=-K_{1}\left[k_{d p} g+\frac{k_{d p}}{M_{p}} f-y_{r e f}^{(2)}-v\right]+k_{d} g+\frac{k_{d}}{M_{r}} f-y_{r e f}^{(2)}
$$

Ordenando e fatorando a equação anterior:

$\dot{z}_{2}=-K_{1}\left(k_{d p}-\frac{k_{d}}{K_{1}}\right) g-K_{1}\left(\frac{k_{d p}}{M_{p}}-\frac{k_{d}}{K_{1} M_{r}}\right) f-K_{1}\left(-1+\frac{1}{K_{1}}\right) y_{r e f}^{(2)}+K_{1} v$

Considerando:

$$
\begin{gathered}
v_{0}=\left(k_{d p}-\frac{k_{d}}{K_{1}}\right) g \\
v_{1}=\left(\frac{k_{d p}}{M_{p}}-\frac{k_{d}}{K_{1} M_{r}}\right) f \\
v_{2}=\left(-1+\frac{1}{K_{1}}\right) y_{\text {ref }}^{(2)}
\end{gathered}
$$

Obtemos

$$
\dot{z}_{2}=K_{1} v-K_{1} v_{0}-K_{1} v_{1}-K_{1} v_{2}
$$

Então teremos o seguinte sistema em malha fechada 


$$
\left\{\begin{array}{l}
\dot{z}_{1}=z_{2} \\
\dot{z}_{2}=K_{1}\left(v-v_{0}-v_{1}-v_{2}\right)=z_{3}
\end{array}\right.
$$

Logo, derivando-se $z_{3}=\dot{z}_{2}$ teremos (considerando a força externa constante e $\left.y_{\text {ref }}^{(3)}=0\right)$

$$
\left\{\begin{array}{l}
\dot{z}_{1}=z_{2} \\
\dot{z}_{2}=z_{3} \\
\dot{z}_{3}=K_{1} \dot{v}=K_{1} w \quad(\text { onde } \quad w=\dot{v})
\end{array}\right.
$$

Assim a lei de controle estabilizante é

$$
\dot{v}=w
$$

n $\quad$ Onde $w=F z, \quad F=\left(-f_{1}-f_{2}-f_{3}\right)$ e $z=\left(z_{1} z_{2} z_{3}\right)^{T}$ fornece o seguinte sistema em malha fechada como visto na figura 4.6.

$$
\left(\begin{array}{c}
\dot{z}_{1} \\
\dot{z}_{2} \\
\dot{z}_{3}
\end{array}\right)=\left(\begin{array}{ccc}
0 & 1 & 0 \\
0 & 0 & 1 \\
-f_{1} & -f_{2} & -f_{3}
\end{array}\right)\left(\begin{array}{l}
z_{1} \\
z_{2} \\
z_{3}
\end{array}\right)
$$

Claramente a escolha de $f_{1}, f_{2}$ e $f_{3}$ impõe pólos que são as raizes de

$$
\Pi(s)=s^{3}+f_{3} s^{2}+f_{2} s+f_{1}
$$


de modo que, para impor tres pólos em $-p$ faremos

$$
f_{1}=p^{3}, \quad f_{2}=3 p^{2}, \quad f_{3}=3 p
$$

Para simular o efeito do desconhecimento de $K_{1}$ na estabilidade em malha fechada pode-se considerar o lugar geométrico das raizes do sistema para $K_{1}$ variando no intervalo $\langle 0, \infty\rangle$, ver figura 4.5

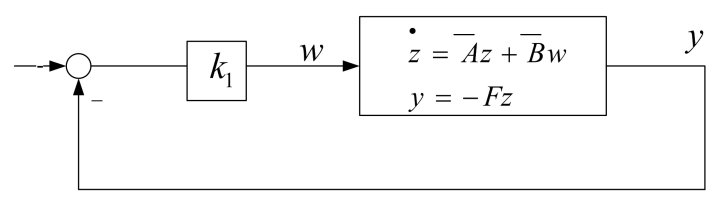

Figura 4.5: Diagrama do sistema em malha fechada

onde as matrizes do sistema são dadas por

$$
\bar{A}=\left(\begin{array}{lll}
0 & 1 & 0 \\
0 & 0 & 1 \\
0 & 0 & 0
\end{array}\right), \quad \bar{B}=\left(\begin{array}{l}
0 \\
0 \\
1
\end{array}\right), \quad \bar{C}=-F
$$

O lugar das raízes em questão é mostrado na figura 4.6. Note que para $K_{1}$ suficientemente grande o sistema em malha fechada é sempre estável.

Agora assuma que $G_{m a}$ denota a função de tranferencia em malha aberta dada por:

$$
G_{m a}=\frac{K_{1}\left(3 p s^{2}+3 p^{2} s+1\right)}{s^{3}}
$$




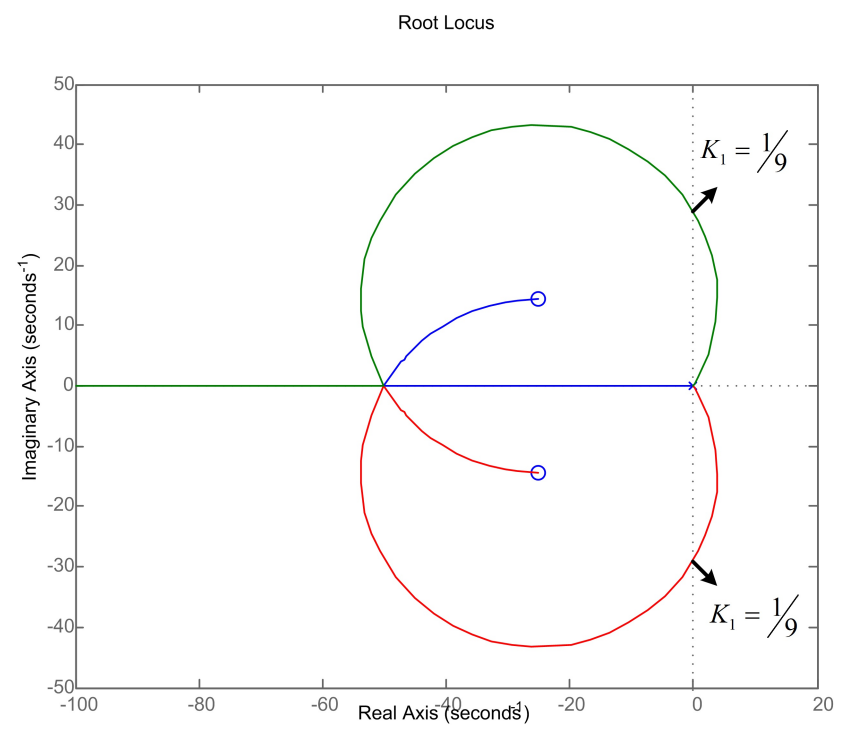

Figura 4.6: Gráfico do lugar das raizes

Logo fechando a malha obtemos:

$$
G_{m f}(s)=\frac{K_{1}\left(3 p s^{2}+3 p^{2} s+p^{3}\right)}{s^{3}+3 p K_{1} s^{2}+3 p^{2} K_{1} s+p^{3} K_{1}}
$$

Utilizando o critério de estabilidade de Routh

$$
\begin{aligned}
& s^{3} \quad 1 \quad 3 p^{2} K_{1} \\
& s^{2} \quad 3 p K_{1} \quad p^{3} K_{1} \\
& \begin{array}{lll}
s^{1} & b_{1} & b_{2}
\end{array} \\
& \begin{array}{lll}
s^{0} & c_{1} & c_{2}
\end{array}
\end{aligned}
$$

Obtemos:

$$
\begin{gathered}
b_{1}=\frac{9 p^{2} K_{1}-p^{2}}{3} \\
c_{1}=p^{3} K_{1}
\end{gathered}
$$


Como $b_{1}$ e $c_{1}$ são elementos da primeira coluna, ambos precissam ser maiores que zero, isto é:

$$
\frac{9 p^{2} K_{1}-p^{2}}{3}>0 \quad \Rightarrow \quad K_{1}>\frac{1}{9}
$$

$$
K_{1}>0
$$

Então os valores que podem fazer com que o sistema seja estavel são $K_{1}>\frac{1}{9}$

Lembrando que a lei de controle estabilizante é dada pela equação (4.6) e a linearizante é dada pela equação (4.5). Esta lei de controle pode ser visualizada no diagrama dado na figura 4.7. Nas simulações feitas na seguinte seção aplicaremos um ruído aditivo gerado por computador como mostrado na figura 4.9.

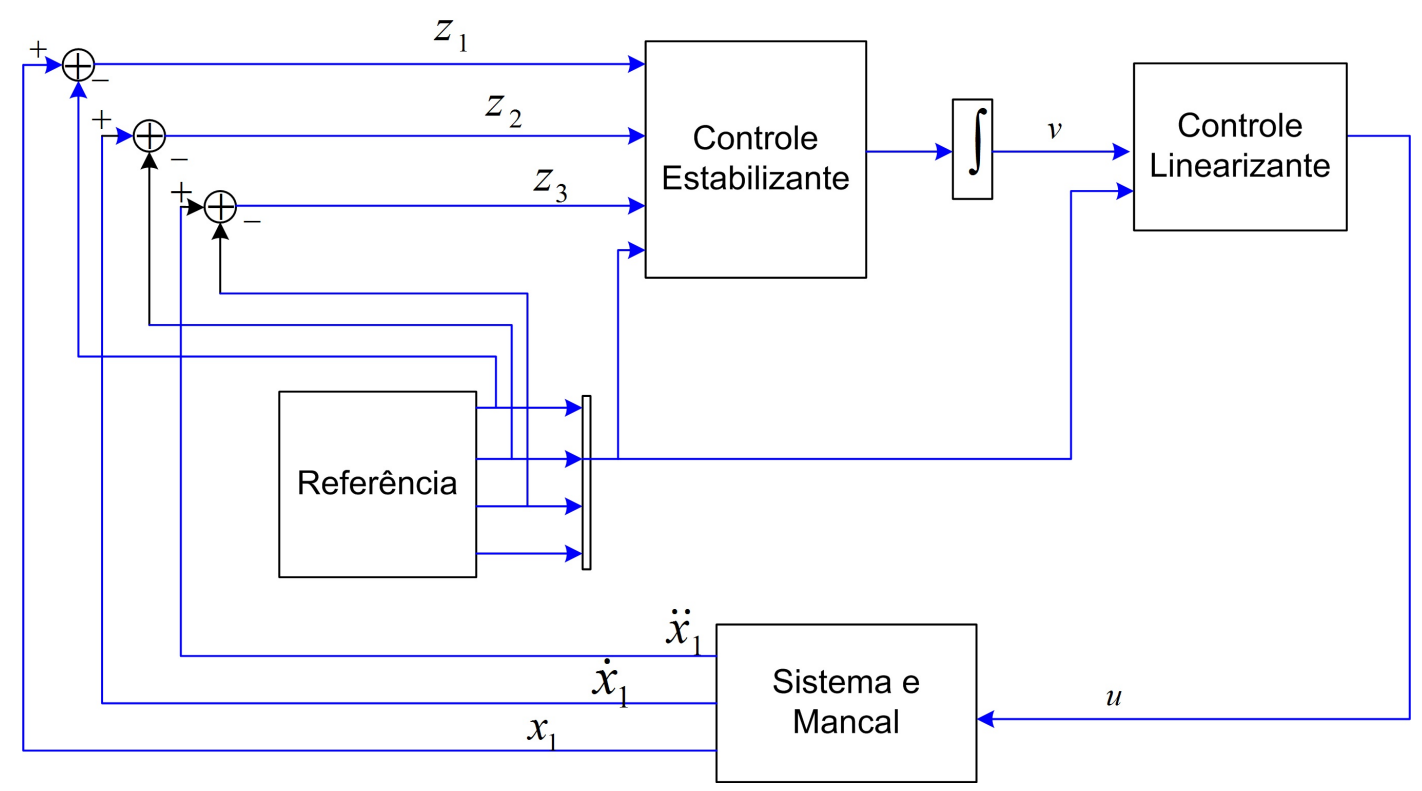

Figura 4.7: Diagrama do sistema de controle em malha fechada 


\subsection{Controle via Linearização Exata com Esti- madores Algebricos (LEA)}

Note que a implementação de tal lei de controle necessita da informação da primeira e da segunda derivadas de $z_{1}(t)$. O controle será então implementado usando os estimadores algébricos correspondentes como visto na figura 4.8. Nas simulações aplicaremos também um ruído aditivo gerado por computador mostrado na figura 4.9. Note que se a estimação for perfeita teremos em malha fechada um sistema equivalente dado na figura 4.7, o esquema implementado é então equivalente a substituir $\dot{z}_{1}$ e $\ddot{x}_{1}$ pelas devidas estimativas algébricas, garantindo então a estabilidade em malha fechada.

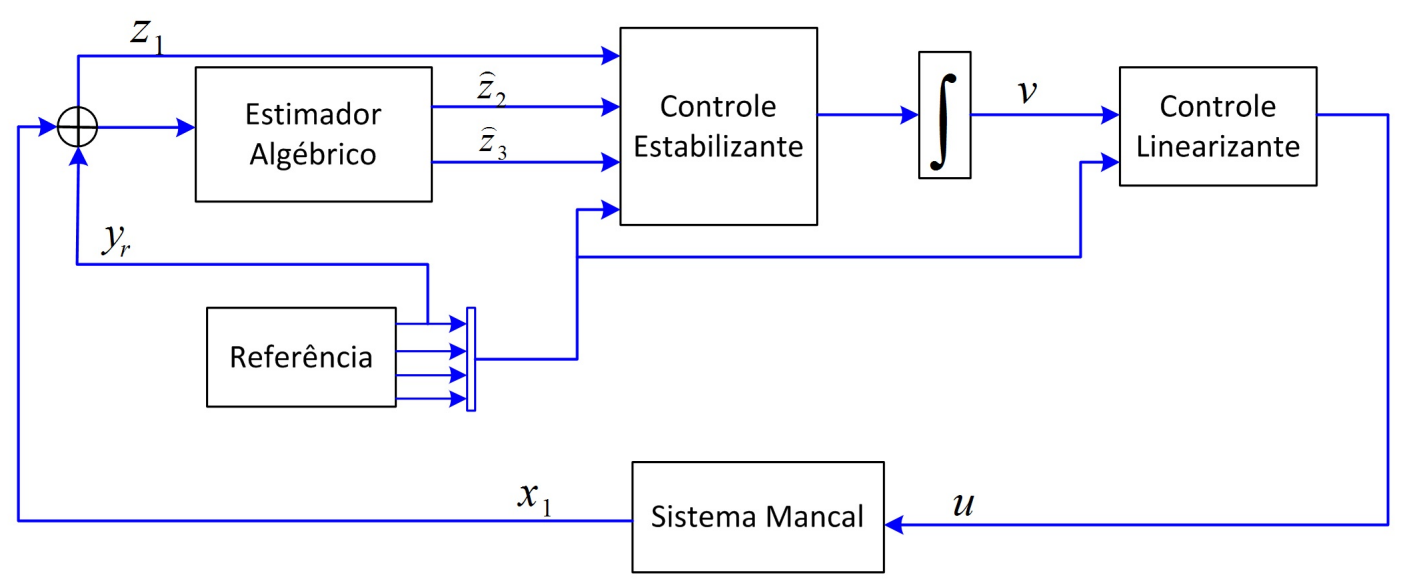

Figura 4.8: Diagrama de estimação e controle em malha fechada 


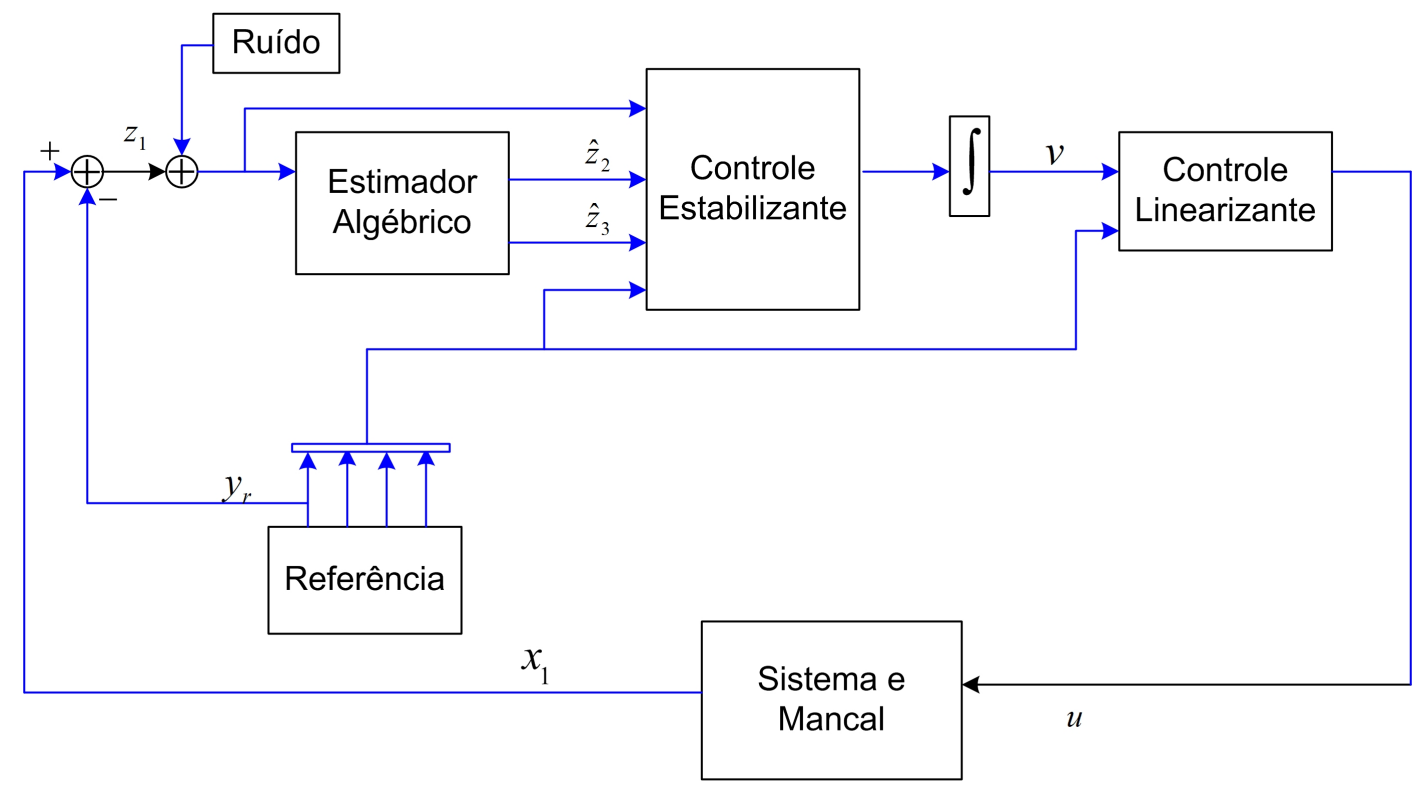

Figura 4.9: Diagrama de estimação e controle em malha fechada com ruido

\subsection{Controle sem termo de gravidade nem de força externa}

Considere agora que, ao invés de utilizar a lei de controle

$$
u= \begin{cases}\eta & \text { se } \eta \geq 0 \\ 0 & \text { se } \eta<0 \quad \text { (região de saturação) }\end{cases}
$$

para $\eta$ dado por (4.4), tomarmos

$$
\eta=\sqrt{\frac{M_{p}}{k_{d p}}\left(\frac{\tilde{x}_{1}}{k_{d p}}\right)^{2} \frac{1}{k_{m p}}\left[-y_{r e f}^{(2)}-v\right]}
$$

Recalculando $\dot{z}_{2}$ como foi feito anteriormente na seção 4.2 


$$
\dot{z}_{2}=\frac{k_{d}}{M_{r}}\left[\frac{-k_{m} u^{2}}{\frac{\tilde{x}_{1}^{2}}{k_{d}^{2}}}+M_{r} g+f\right]-y_{r e f}^{(2)}
$$

Substituindo o valor de $u$, obtemos:

$$
\dot{z}_{2}=\frac{-k_{d}^{3} k_{m} M_{p}}{k_{d p}^{3} k_{m p} M_{r}}\left[-y_{r e f}^{(2)}-v\right]+k_{d} g+\frac{k_{d}}{M_{r}} f-y_{r e f}^{(2)}
$$

Lembrando que $K_{1}=\frac{k_{d}^{3} K_{m} M_{p}}{k_{d p}^{3} k_{m p} M_{r}}$

$$
\begin{gathered}
\dot{z}_{2}=-K_{1}\left[-y_{r e f}^{(2)}-v\right]+k_{d} g+\frac{k_{d}}{M_{r}} f-y_{\text {ref }}^{(2)} \\
\dot{z}_{2}=-K_{1}\left(-\frac{k_{d}}{K_{1}}\right) g-K_{1}\left(-\frac{k_{d}}{k_{1} M_{r}}\right) f-K_{1}\left(-1+\frac{1}{K_{1}}\right) y_{r e f}^{(2)}+K_{1} v
\end{gathered}
$$

Sejam

$$
\begin{gathered}
v_{0}^{\prime}=\left(-\frac{k_{d}}{K_{1}}\right) g \\
v_{1}^{\prime}=\left(-\frac{k_{d}}{K_{1} M_{r}}\right) f \\
v_{2}^{\prime}=\left(-1+\frac{1}{K_{1}}\right) y_{r e f}^{(2)}
\end{gathered}
$$

A tendência é termos $v_{0}^{\prime}$ e $v_{1}^{\prime}$ não nulos enquanto $v_{0}, v_{1}, v_{2}$ seriam nulos para $k_{d p}=k_{d}, M_{p}=M_{r}, k_{m p}=k_{m}$.

A nova expressão de $\dot{z}_{2}$ será: 


$$
\dot{z}_{2}=K_{1}\left(v-v_{0}^{\prime}-v_{1}^{\prime}-v_{2}^{\prime}\right)
$$

onde $v_{0}^{\prime}, v_{1}^{\prime}, v_{2}^{\prime}$ serão constantes, eventualmente diferentes de $v_{0}, v_{1}, v_{2}$. Como estimamos (ou medimos) $\dot{z}_{2}=z_{3}$, a dinâmica em malha fechada com a lei de controle

$$
w=F z
$$

será análoga à obtida anteriormente em (4.7), sendo que a condição inicial de $z_{3}=\dot{z}_{2}$ é que muda. A estratégia de controle funcionará portanto de maneira semelhante a menos dos transitórios.

\subsection{Simulações do controle LE e LEA}

Nesta seção serão apresentados os resultados das simulações obtidas mediante as abordagens de estimação e controle LE e LEA descritas anteriormente. As implementações serão feitas em simulink e utilizarão os valores dados na tabela 4.1. Uma perturbação do tipo degrau é aplicada no tempo $t=2$ segundos, também é aplicado um ruído gerado pelo computador, utilizando Band-Limited White Noise do Matlab/Simulink ${ }^{\circledR}$. Começaremos apresentando as simulações feitas com o sistema flexível, depois o sistema rigído e por último as simulações com o controle sem termo de gravidade e nem de força externa. 


\subsubsection{Modelo flexível (sem ruído)}

\subsubsection{Simulações de desempenho sem ruído do modelo flexível}

Nas figuras 4.10, 4.11 e 4.12 são mostradas comparações do posicionamento, velocidade e aceleração real obtidas mediante as estratégias de LE e LEA do sistema flexível. Observa-se que o comportamento do estimador-controlador LEA é bem próximo do controle LE (estimação perfeita).

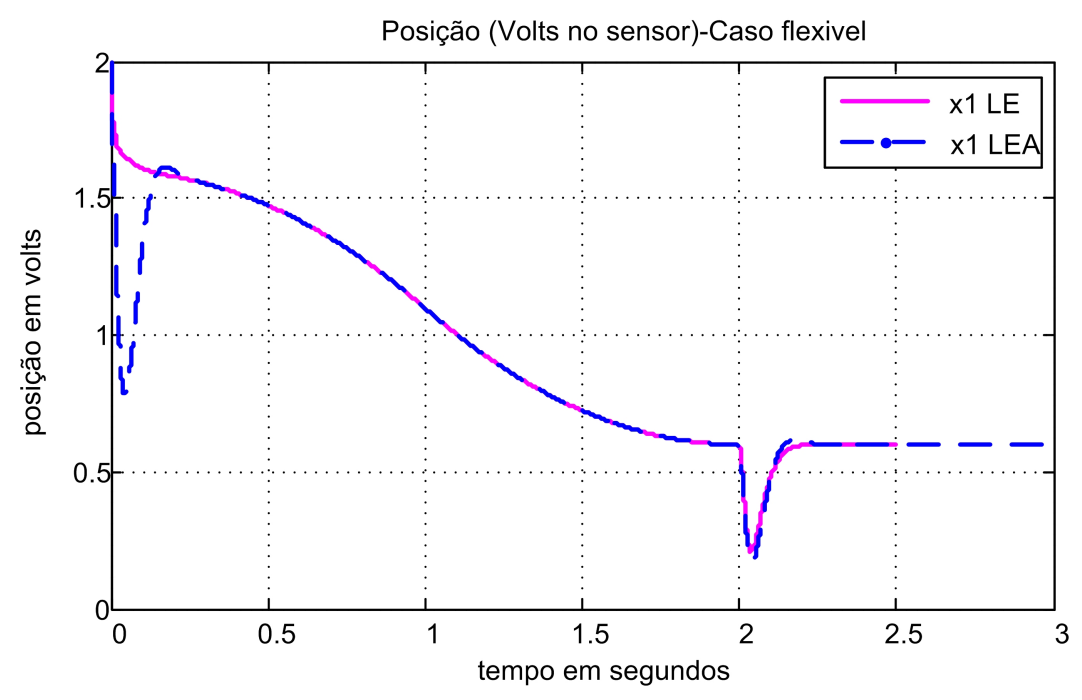

Figura 4.10: Posição real utilizando o controlador LE e LEA.

\subsubsection{Modelo flexível (com ruído)}

\subsubsection{Simulações de desempenho com ruído do modelo flexível}

As figuras 4.13, 4.14 e 4.15 mostram também as comparações do posicionamento, velocidade e aceleração obtidas pelas estratégias LE e LEA do sistema flexível, neste caso afetadas pelo ruído. A figura 4.16 mostra o "zoom" da figura 


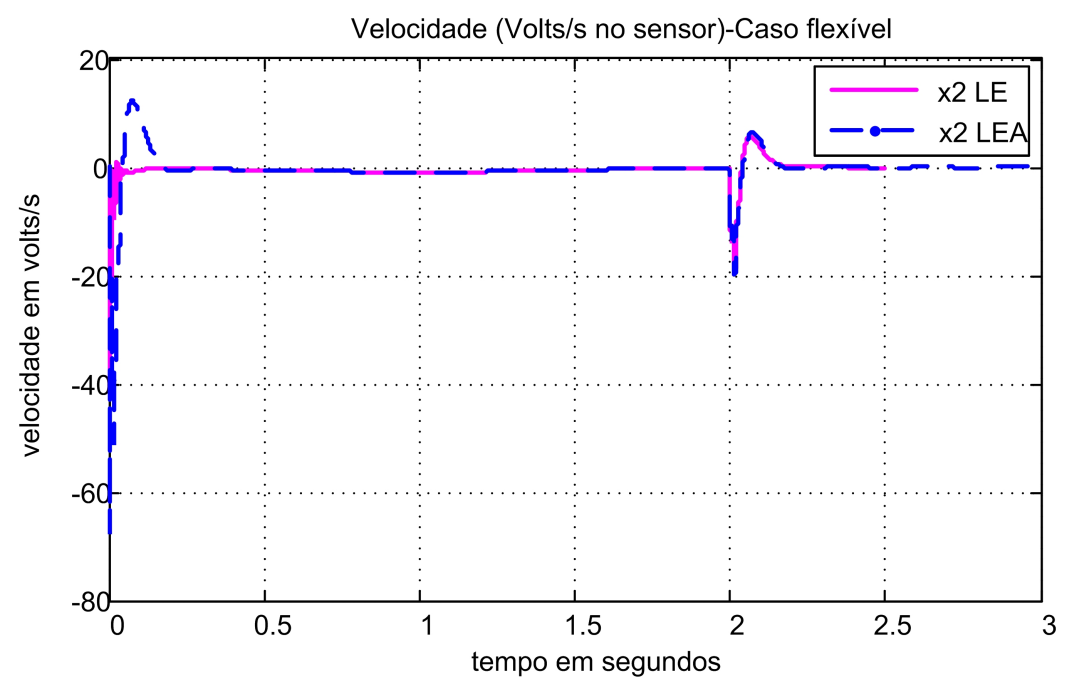

Figura 4.11: Velocidade real utilizando o controlador LE e LEA.

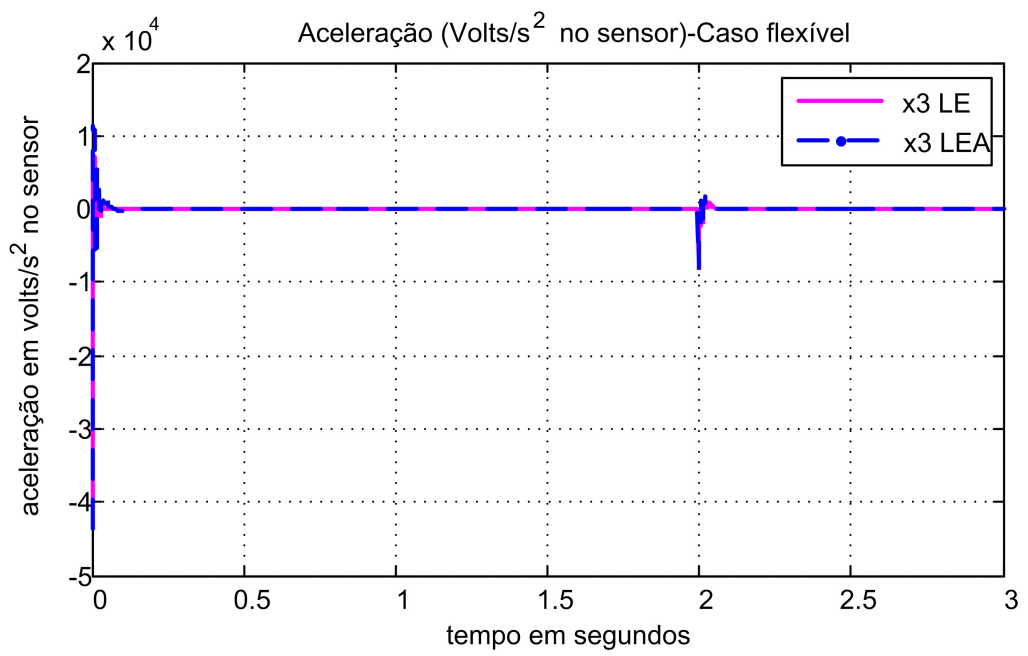

Figura 4.12: Aceleração real utilizando o controlador LE e LEA

4.15 onde a aceleração real $x_{3}$ obtida pelo controle LEA estabiliza no tempo 0.05 segundos aproximadamente, no caso do controle LE estabiliza em 0.04 segundos aproximadamente. 


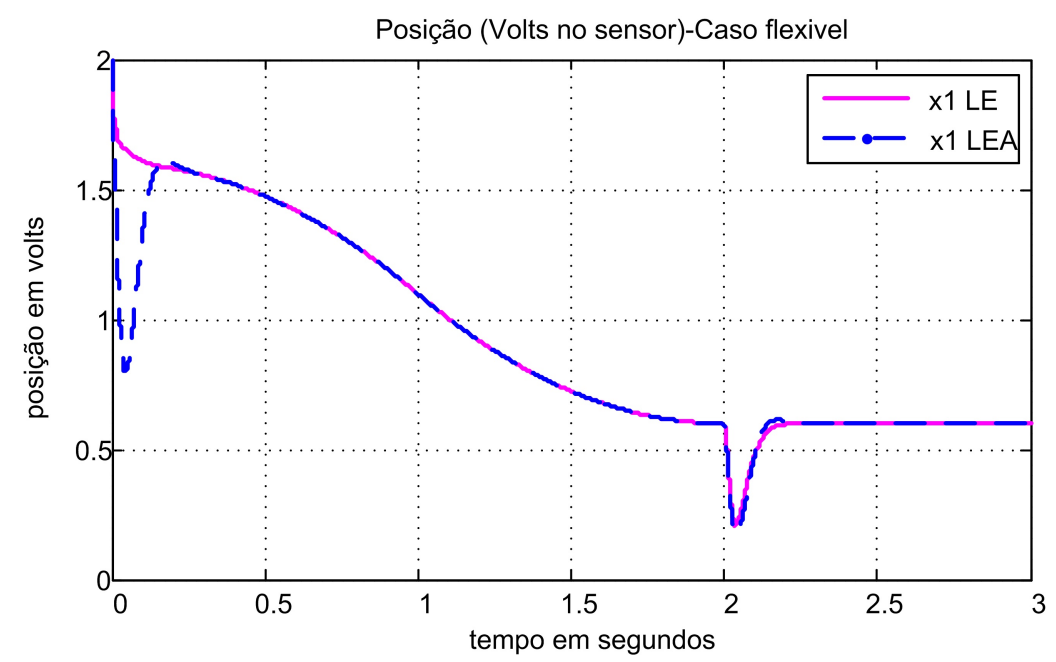

Figura 4.13: Posição real utilizando o controlador LE e LEA com ruído

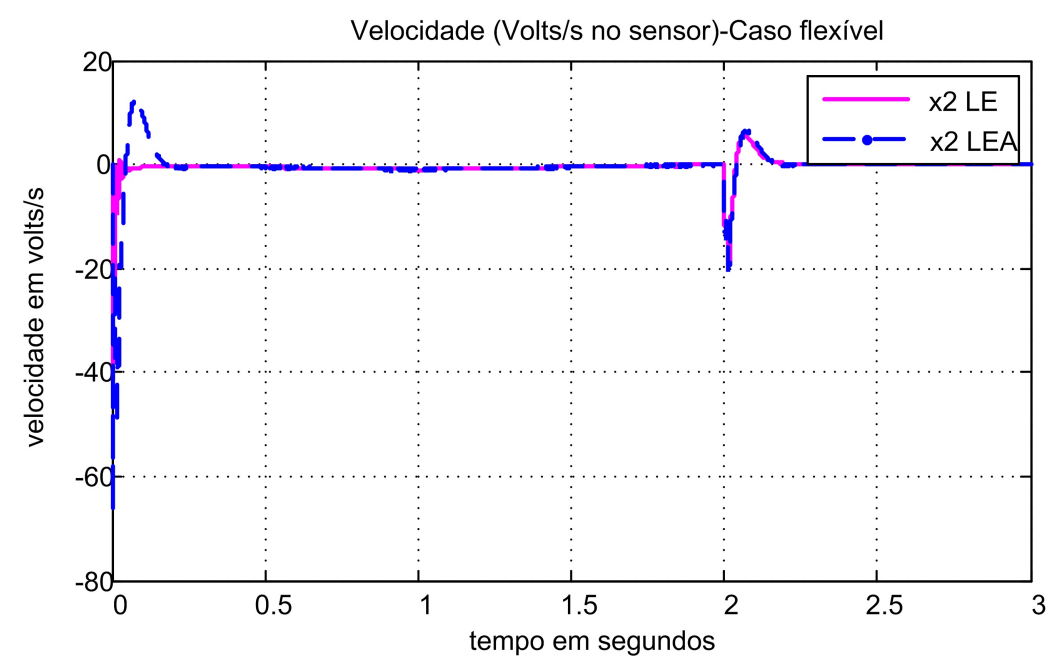

Figura 4.14: Velocidade real utilizando o controlador LE e LEA com ruído

\subsubsection{Erro de rastreamento do modelo flexível}

As figuras 4.17, 4.18 e 4.19 apresentam os erros de rastreamento $z_{1}, z_{2}$ e $z_{3}$. 


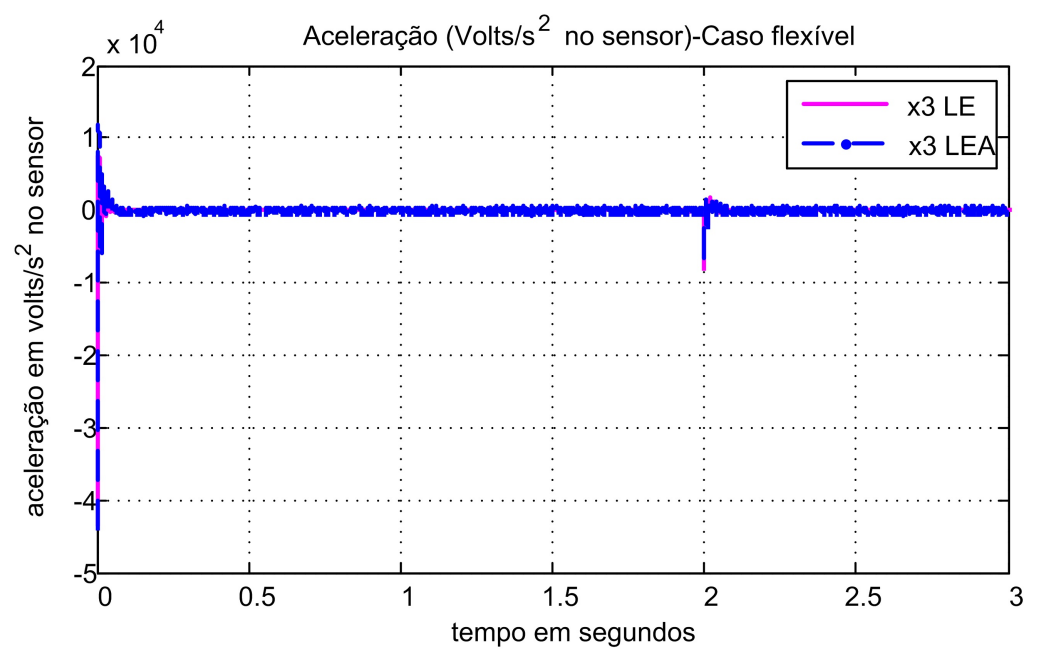

Figura 4.15: Aceleração real utilizando o controlador LE e LEA

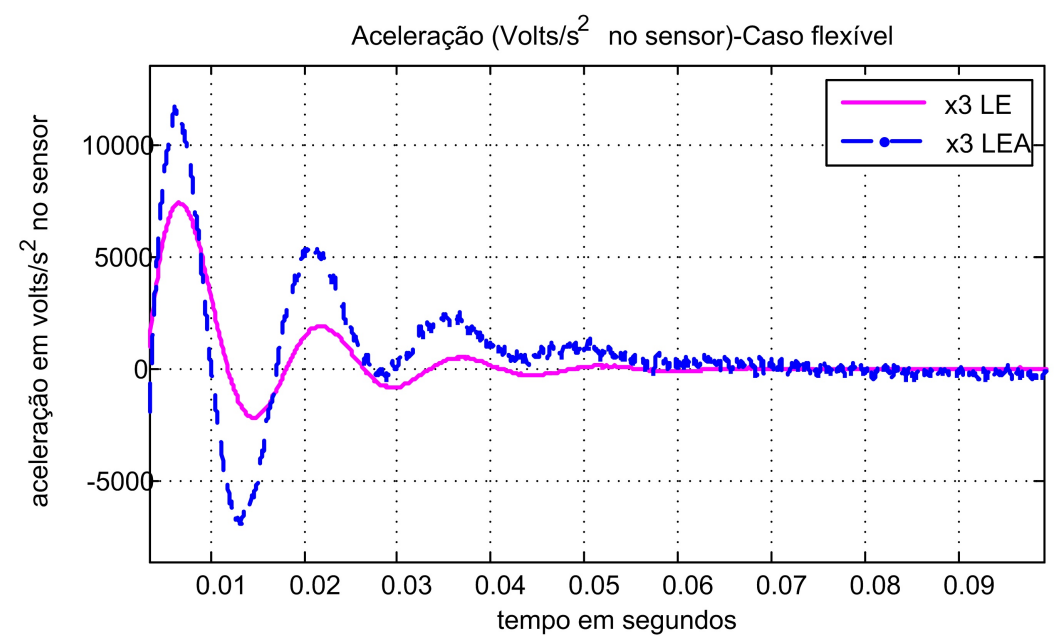

Figura 4.16: Zoom da aceleração real

\subsubsection{Simulação focando o estimador do modelo flexível}

As simulações seguintes mostram alguns resultados obtidos para o sistema de controle LEA no caso flexível, num ambiente ruidoso. As figuras 4.20 e 4.21 apresentam as comparações entre estados reais e os estados estimados. 


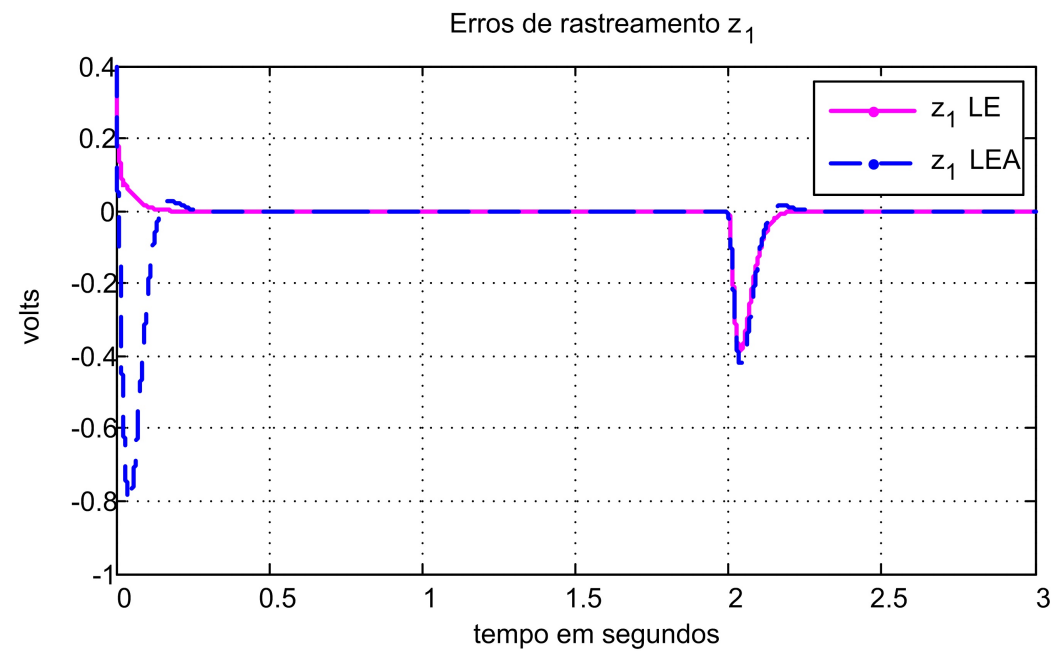

Figura 4.17: Comparação entre os erros de rastreamento $z_{1}$ LE e LEA

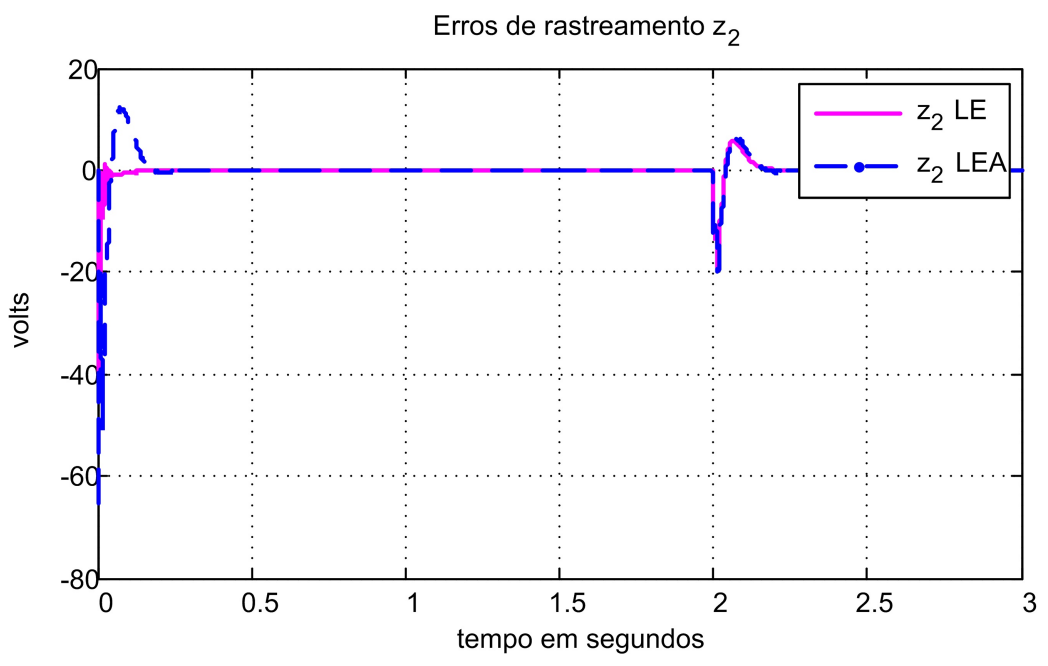

Figura 4.18: Comparação entre os erros de rastreamento $z_{2}$ LE e LEA

\subsubsection{Esforço de controle do modelo flexível}

Apresentamos o esforço de controle mostrado na figura 4.22 das abordagens LE e LEA. 


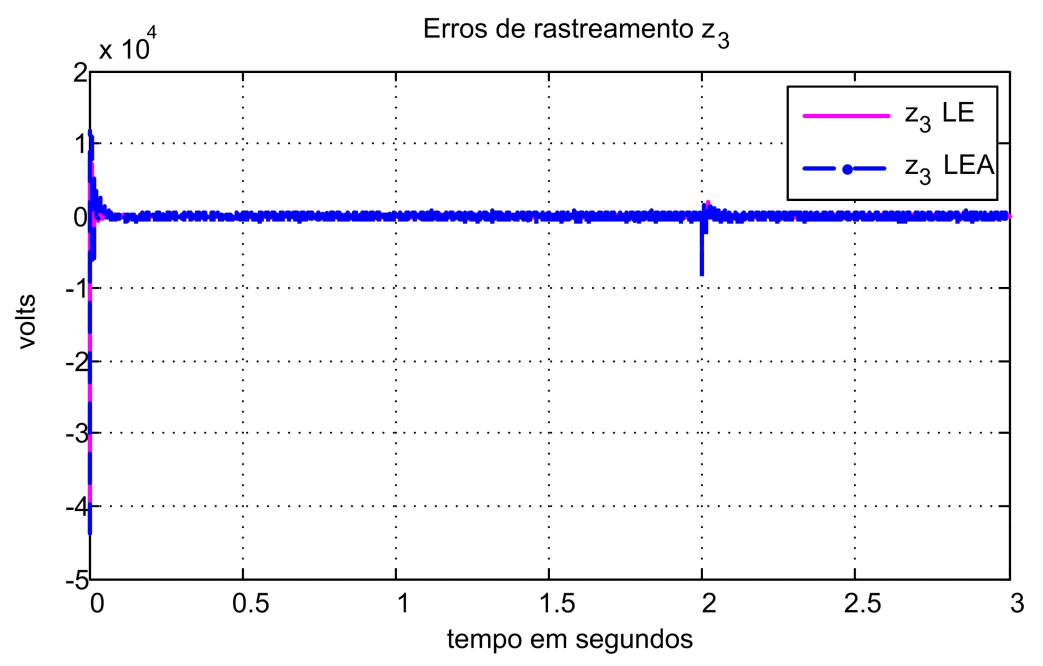

Figura 4.19: Comparação entre os erros de rastreamento $z_{3}$ LE e LEA

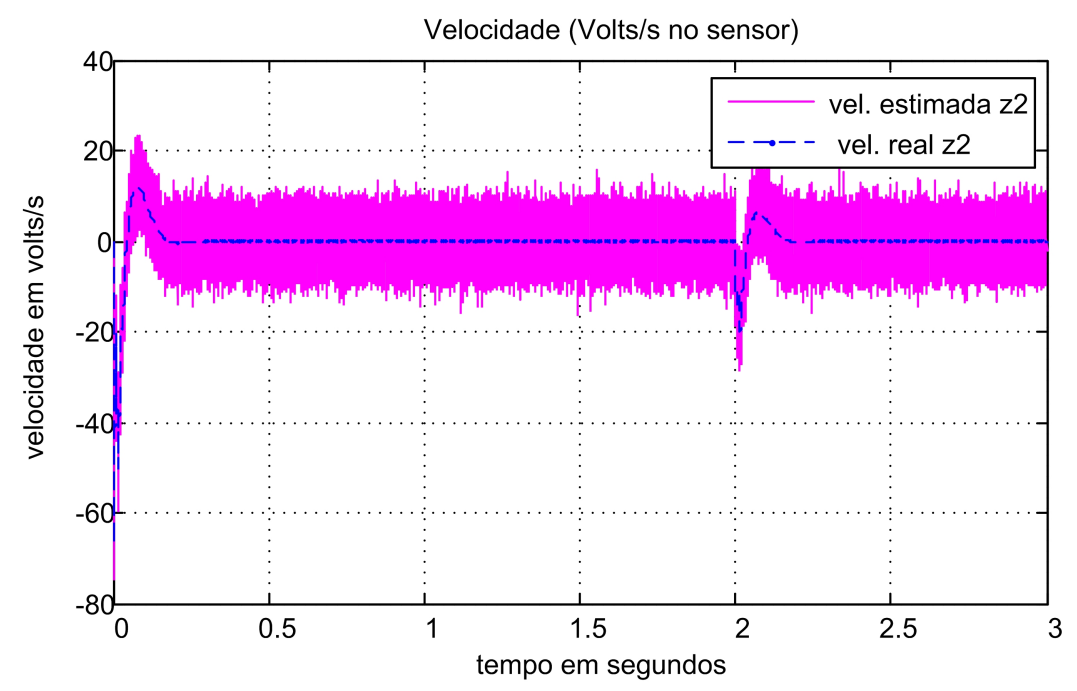

Figura 4.20: Estado estimado e estado real (velocidade)

\subsubsection{Modelo Rígido (com ruído)}

\subsubsection{Desempenho com ruído do modelo rígido}

As seguintes simulações são feitas com o modelo rígido e com adição de ruído. 


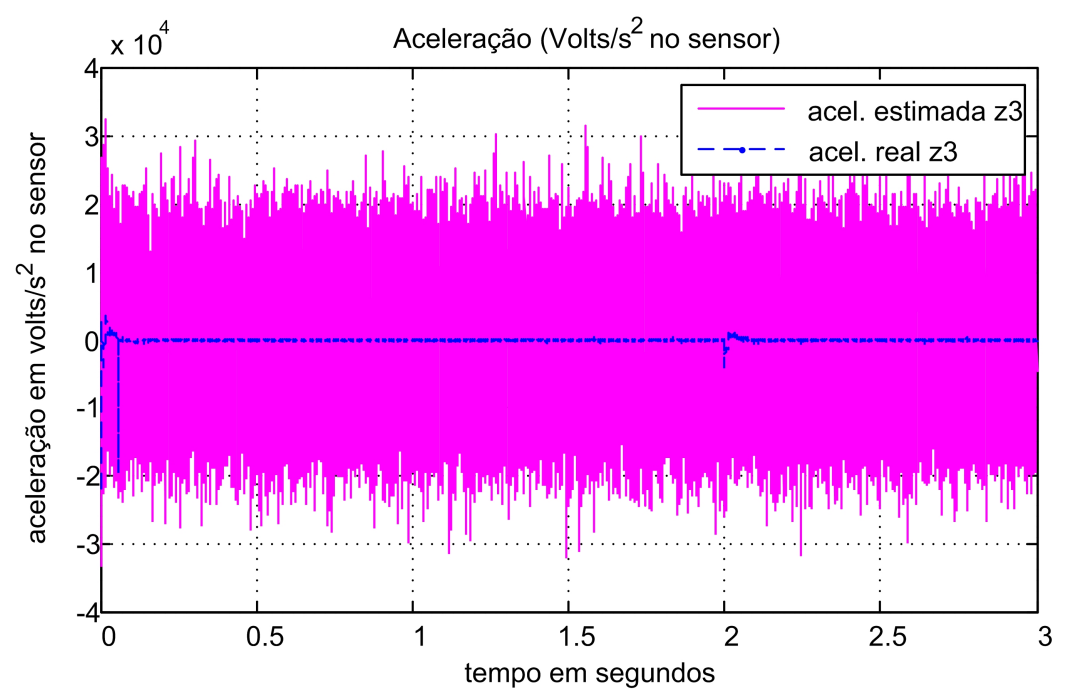

Figura 4.21: Estado estimado e estado real (aceleração)

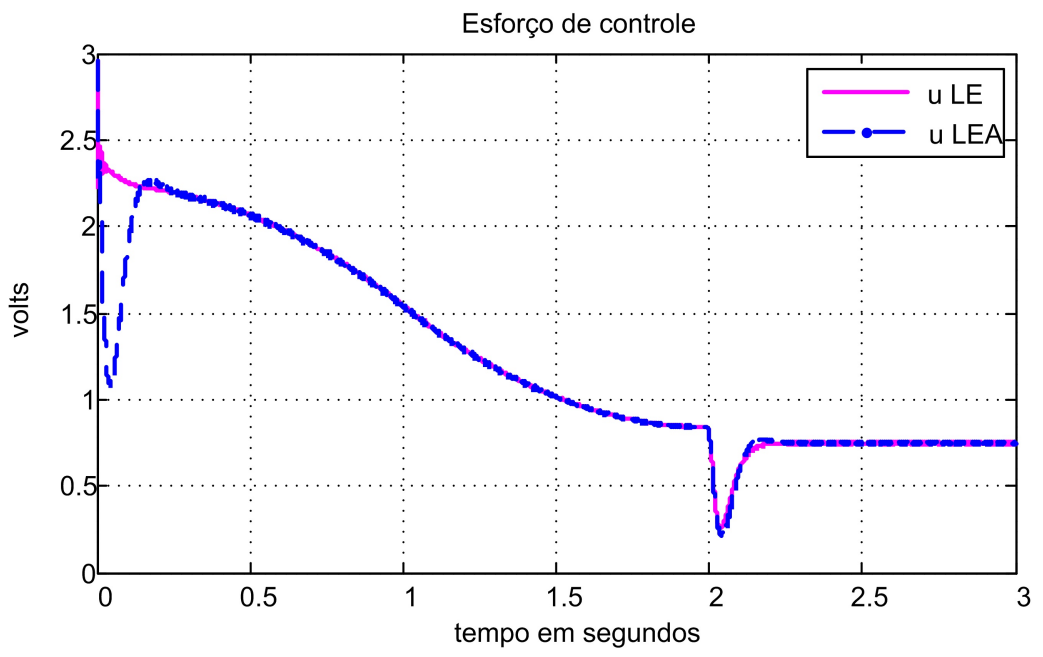

Figura 4.22: Comparação entre os esforços de controle LE e LEA

\subsubsection{Simulações do controle da seção 4.4}

Nesta seção são apresentada simulações do modelo flexível em ambiente ruidoso, especificamente do controle sem termo de gravidade nem força externa como mostra a seção 4.4 . 


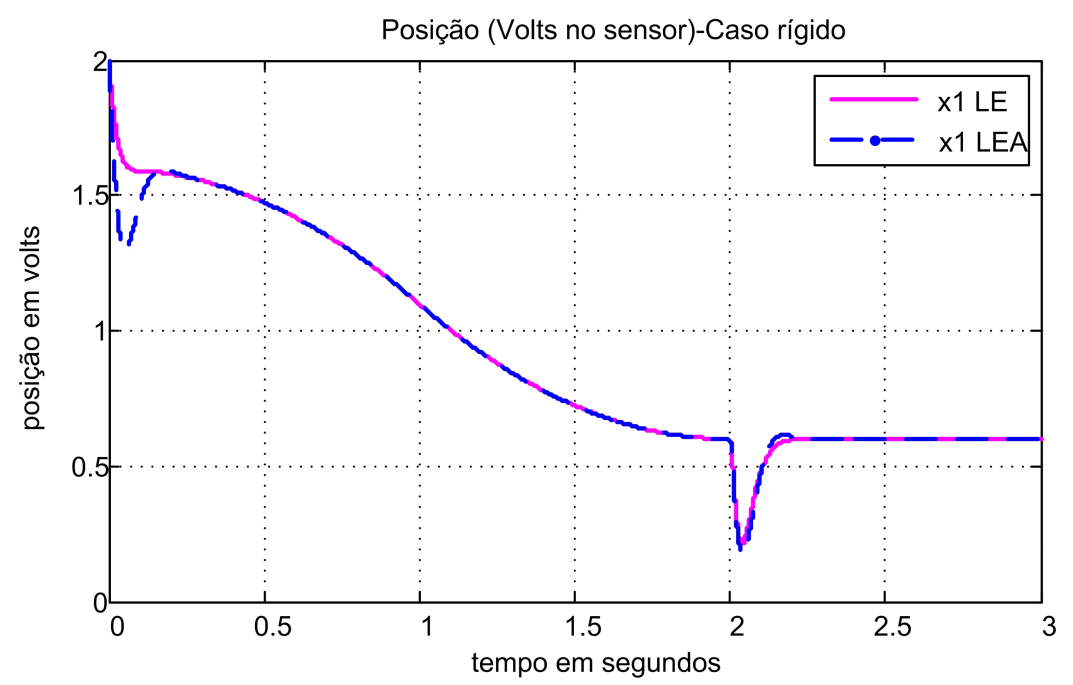

Figura 4.23: Posição real do modelo rígido utilizando o controlador LE e LEA com ruído

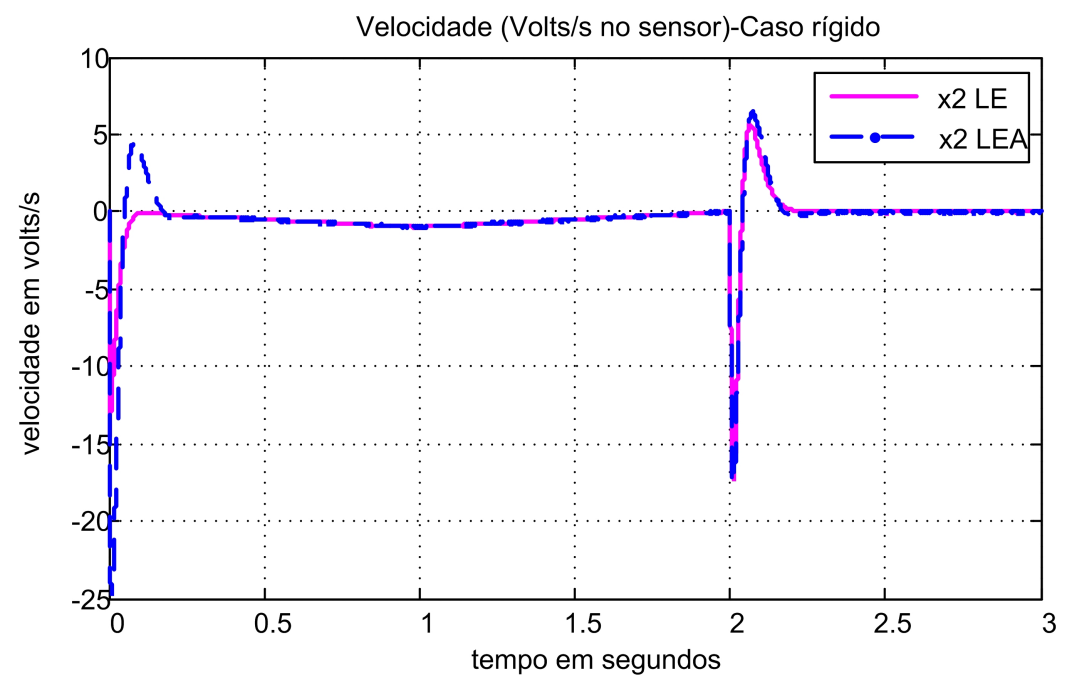

Figura 4.24: Velocidade real do modelo rígido utilizando o controlador LE e LEA.

\subsubsection{Simulaçao do erro de rastreamento}

As figuras 4.29, 4.30 e 4.31 apresentam os erros de rastreamento $z_{1}, z_{2}$ e $z_{3}$. 


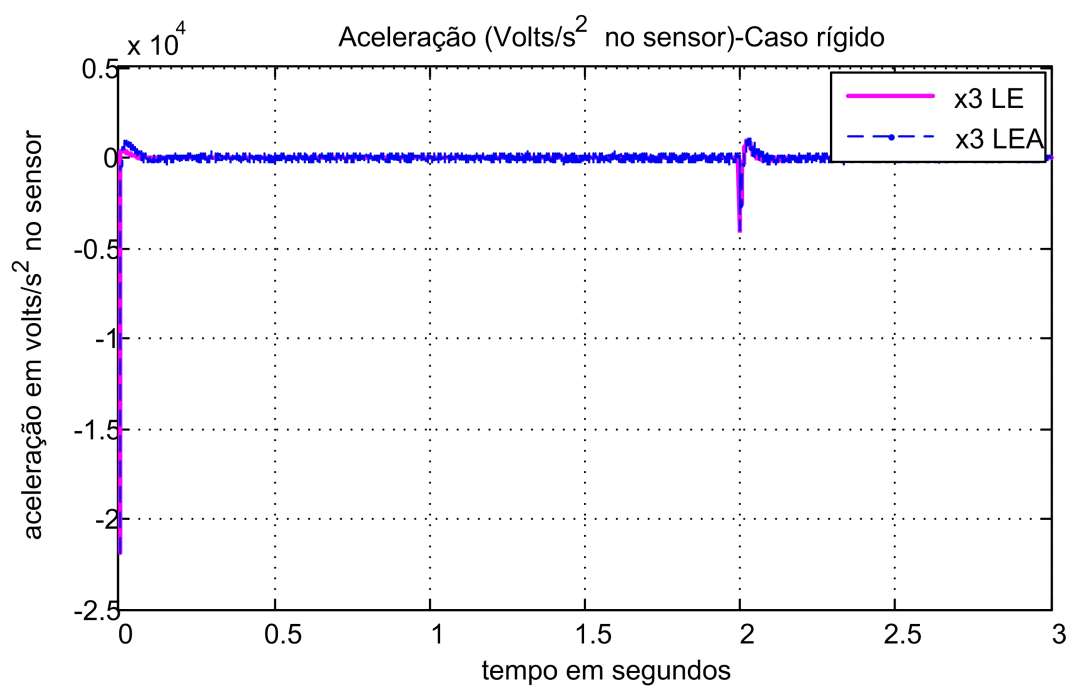

Figura 4.25: Aceleração real do modelo rígido utilizando o controlador LE e LEA.

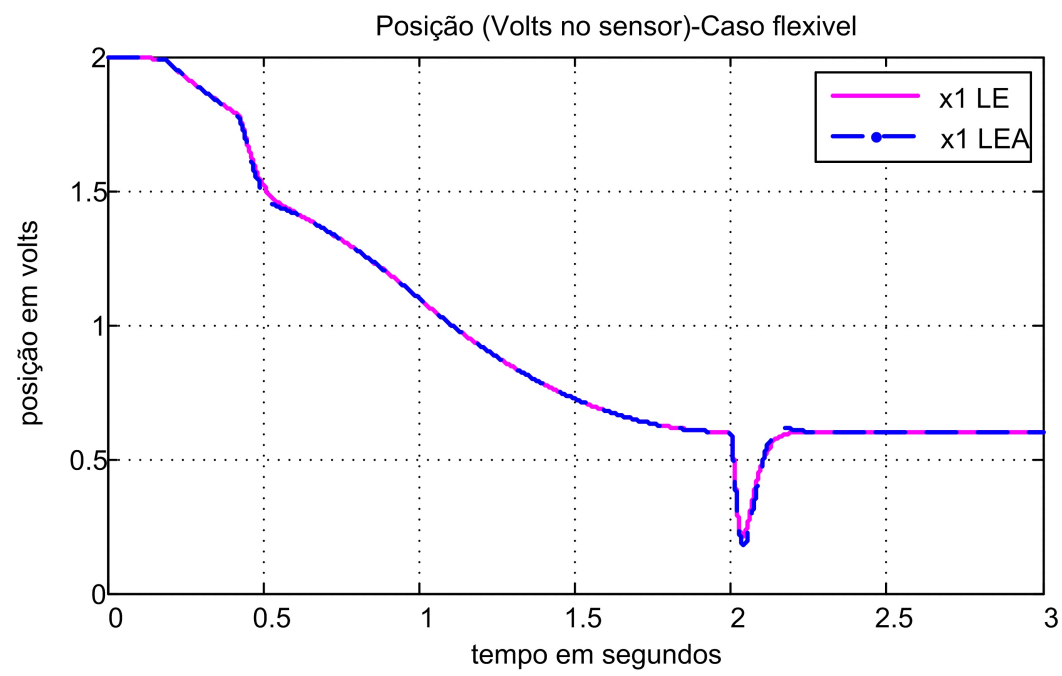

Figura 4.26: Posição real utilizando o controlador LE e LEA com ruído

\subsubsection{Esforço de controle}

Apresentamos o esforço de controle mostrado na figura 4.32 das abordagens LE e LEA. 


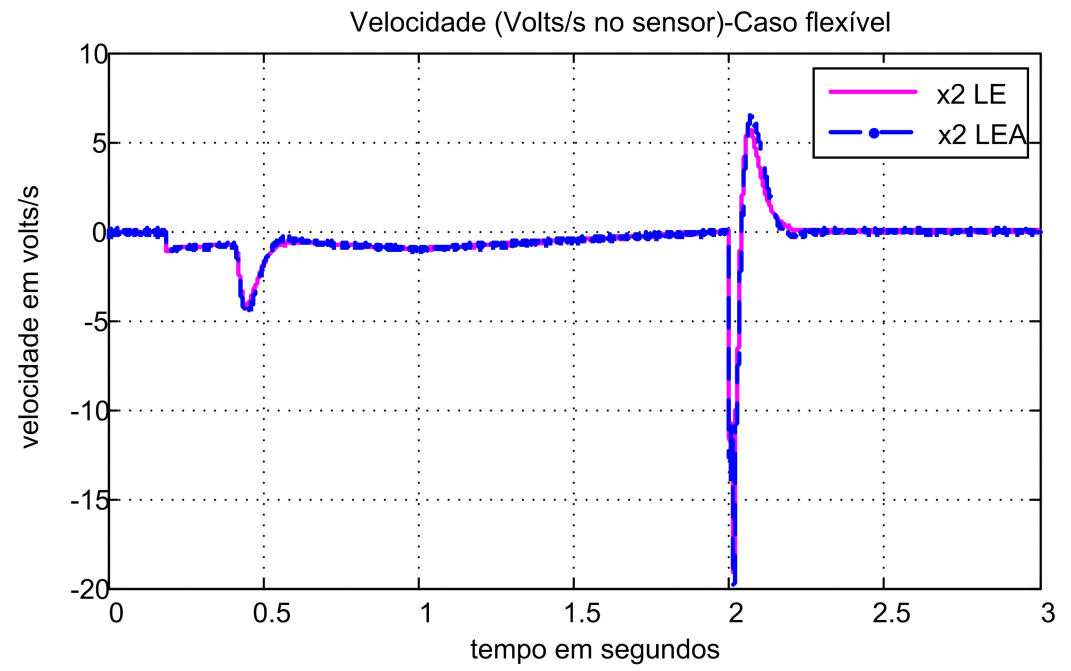

Figura 4.27: Velocidade real utilizando o controlador LE e LEA com ruído

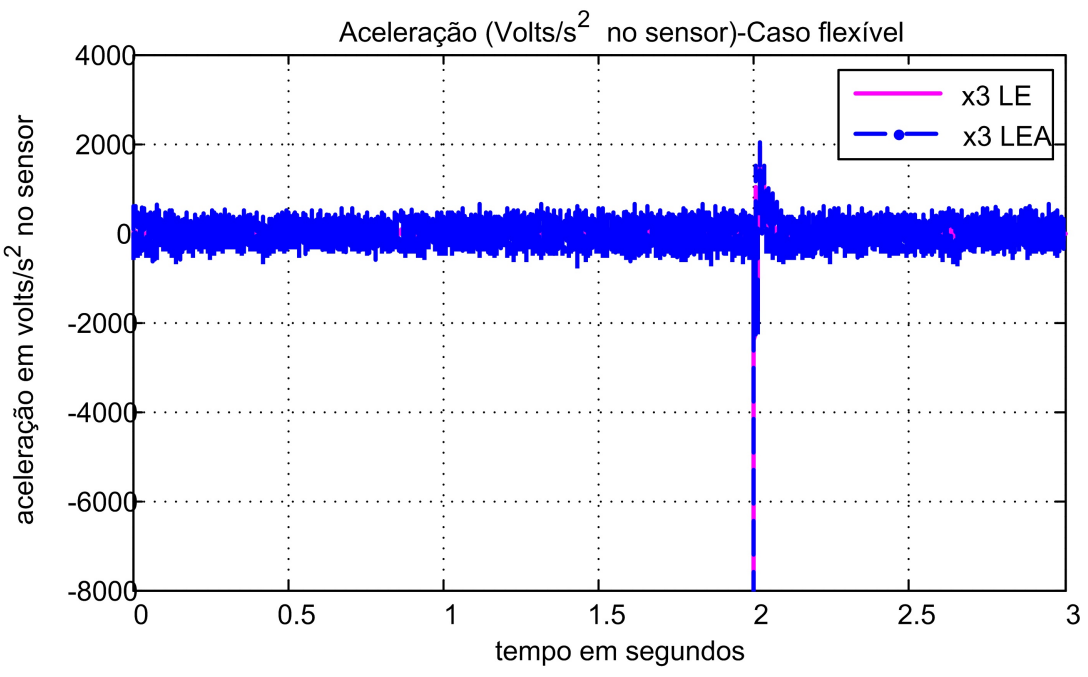

Figura 4.28: Aceleração real utilizando o controlador LE e LEA com ruído 


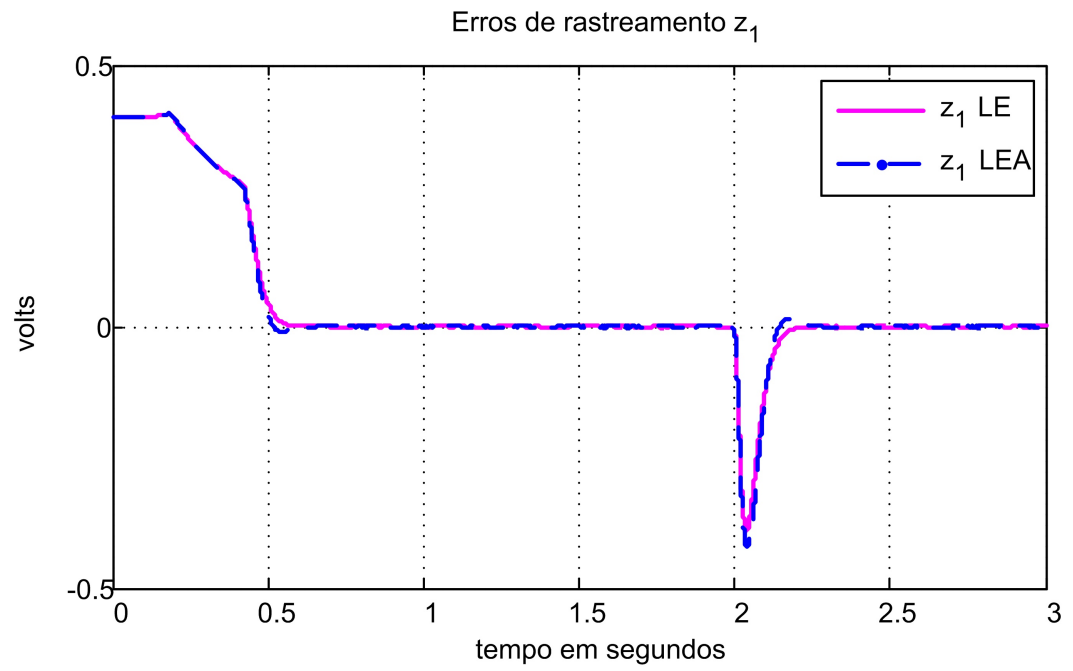

Figura 4.29: Comparação entre os erros de rastreamento $z_{1}$ LE e LEA

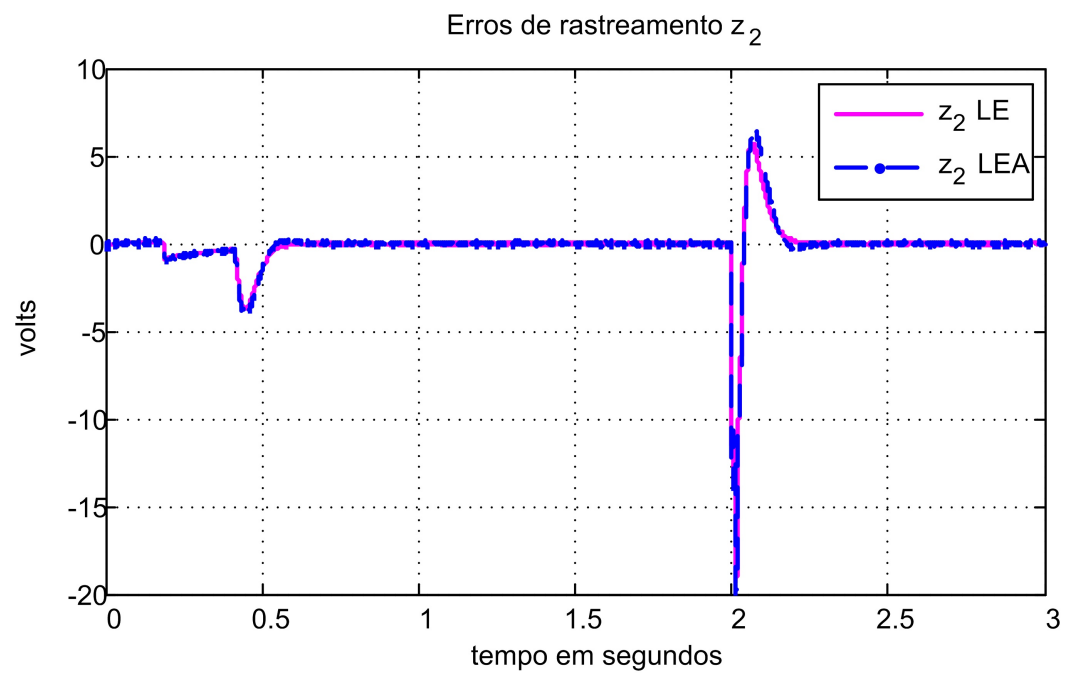

Figura 4.30: Comparação entre os erros de rastreamento $z_{2}$ LE e LEA 


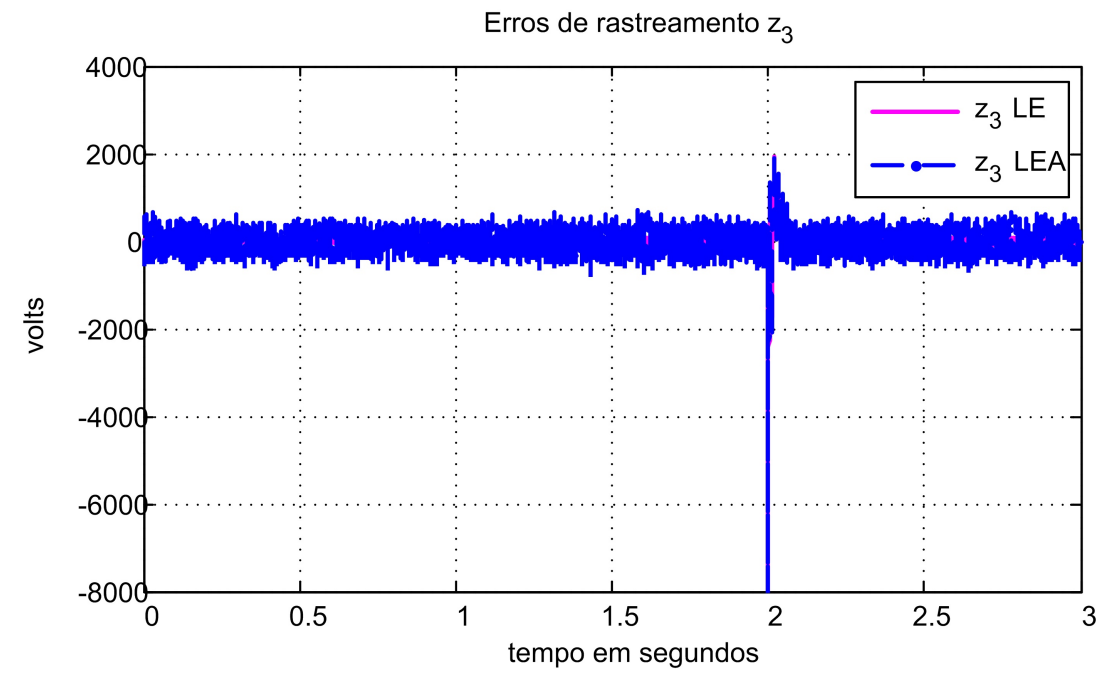

Figura 4.31: Comparação entre os erros de rastreamento $z_{3}$ LE e LEA

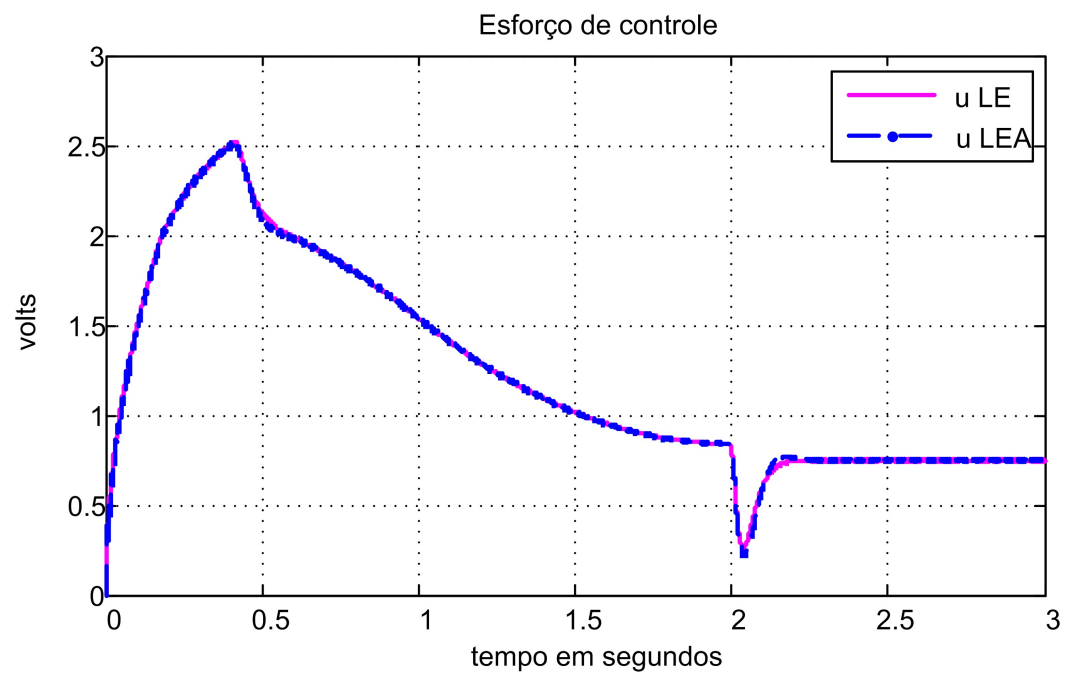

Figura 4.32: Comparação entre os esforços de controle LE e LEA 


\section{Capítulo 5}

\section{CONSIDERAÇÕES FINAIS}

Este trabalho é a continuação de minha dissertação de mestrado (Murgueytio 2011), (Murgueytio, Silva e Novaes 2013) que desenvolveu um estudo de casos, aplicando a estimação algébrica em malha fechada para o controle de um pêndulo invertido. Em (Murgueytio 2011), (Murgueytio, Silva e Novaes 2013) avaliamos o com-

promisso de precisão, robustez e rejeição a ruído num contexto heurístico. A presente tese considerou os aspectos formais da teoria e diversos resultados matemáticos foram demonstrados:

- Expressões teóricas para a precisão do estimador de ordem 2 e 3 foram obtidas mostrando-se que a mesma pode ser arbitrariamente grande quando se faz o valor de $h$ ser arbitrariamente pequeno.

- Foram demostrados teoremas de estabilidade em malha fechada com estimadores de ordem 2 e 3 , tendo a expressão do erro de estimação como ferramenta fundamental.

Embora exista uma semelhança entre os teoremas aqui demonstrados, com o 
princípio da separação, a prova destes resultados não é da mesma natureza das demonstrações tradicionais, visto que a dinâmica do estimador não é levada em conta, mas apenas a estimativa do erro cometido. Mais ainda, o resultado vale para qualquer estimador que respeite os mesmos limites de erro. A aplicação dos estimadores algébricos em malha fechada foi implementada digitalmente para controlar um sistema de levitação magnética. Foram obtidos excelentes resultados combinando-se os estimadores algébricos de segunda e terceira ordem com a linearização exata. 


\section{Referências Bibliográficas}

Ahrens e Khalil 2009 AHRENS, J.; KHALIL, H. High-gain observers in the presence of measurement noise: A switched-gain approach. Automatica, v. 45, p. 936-943, 2009.

Atassi e Khalil 1999 ATASSI, A.; KHALIL, H. A separation principle for the stabilization of a class of nonlinear systems. IEEE Trans. Automat. Contr., v. 44, p. $1672-1687,1999$.

Atassi e Khalil 2000 ATASSI, A.; KHALIL, H. Separation results for the stabilization of nonlinear systems using different high-gain observer designs. Syst. \& Control Letters, v. 39, p. 183-191, 2000.

Atassi e Khalil 2001 ATASSI, A.; KHALIL, H. A separation principle for the control of a class of nonlinear systems. IEEE Trans. Automat. Contr., v. 46, p. 742-746, 2001.

Chen 1999 CHEN, C. T. Linear system theory and design. 3. ed. New York: Oxford University Press, 1999.

Cunha et al. 2005 CUNHA, J. P. V. S. et al. Controle de sistemas lineares incertos por modos deslizantes e observador de alto ganho sem peaking. Revista Controle \& Automação (SBA), v. 16, p. 449-466, 2005.

Diop e Fliess 1991 DIOP, S.; FLIESS, M. On nonlinear observability. In: ECC'91. [S.l.: s.n.], 1991. p. 152-157.

Diop, Grizzle e Chaplais 2000 DIOP, S.; GRIZZLE, J. W.; CHAPLAIS, F. On numerical differentiation algorithms for nonlinear estimation. In: IEEE CDC'2000. [S.l.: s.n.], 2000.

Diop et al. 1994 DIOP, S. et al. Interpolation and numerical differentiation for observer desing. In: 1994 American Control Conference. Baltimore: [s.n.], 1994. p. 1329-1333. 
Fliess e Sira-Ramirez 2004 FLIESS, M.; SIRA-RAMIREZ, H. Control via state estimations of some nonlinear systems. In: IFAC Nolcos Conference. Stutgart, Germany: [s.n.], 2004.

Fliess e Sira-Ramirez 2004 FLIESS, M.; SIRA-RAMIREZ, H. Reconstructeurs d'etats. C.R. Acad. Sci. Paris: I, v. 338, p. 91-96, 2004.

Ibrir e Diop 2004 IBRIR, S.; DIOP, S. A numerical procedure for filtering and efficient high-order signal differentiation. Int. J. Appl. Math. Compt. Sci, v. 14, p. 201-208, 2004.

Khalil 1996 KHALIL, H. K. Nonlinear Systems. 2. ed. Upper Saddle River, New Jersey: Prentice-Hall, 1996.

Levant 2003 LEVANT, A. Higher-order sliding modes, differentiation and output-feedback control. Int. J. Control, v. 76, p. 924-941, 2003.

Lima 1981 LIMA, E. L. Curso de Análise, vol. 2. Rio de janeiro: IMPA Projeto Euclides, 1981. 558 p.

Mboup, Join e Fliess 2009 MBOUP, M.; JOIN, C.; FLIESS, M. Numerical differentiation with annihilators in noisy environment. Numerical Algorithms, v. 50, n. 4, p. 439-467, 2009.

Moraes e Silva 2015a MORAES, M. S.; SILVA, P. S. P. Model-free control of magnetic levitation systems through algebraic derivative estimation. In: COBEM2015. Rio de Janeiro: [s.n.], 2015a.

Moraes e Silva 2015b MORAES, M. S.; SILVA, P. S. P. Algebraic derivative estimation and applications in adaptive control of magnetic levitation systems. In: COBEM2015. Rio de Janeiro: [s.n.], 2015b.

Murgueytio 2011 MURGUEYTIO, Z. V. L. Estimação Algébrica Aplicada aos Sistemas de Controle - Um Estudo de Casos. Dissertação (Mestrado) - Escola Politécnica, 2011.

Murgueytio, Silva e Novaes 2013 MURGUEYTIO, Z. V. L.; SILVA, P. S. P.; NOVAES, C. E. B. Closed-loop performance analysis of algebraic estimators. $J$ Control Autom Electr Syst, v. 24, p. 725-740, 2013.

Novaes 2010 NOVAES, C. E. B. Métodos de estimação de derivadas via cálculo operacional e aplicações a problemas de controle. Dissertação (Mestrado) Escola Politécnica, 2010. 
Novaes e Silva 2009 NOVAES, C. E. B.; SILVA, P. S. P. On non-asymptotic state observers and their implementation in discrete time. In: DINCON'09. Bauru: [s.n.], 2009. p. 45-53.

Ogata 1982 OGATA, K. Engenharia de Controle Moderno. Rio de Janeiro: Prentice-Hall do Brasil, 1982.

Oliveira, Peixoto e Hsu 2010 OLIVEIRA, T. R.; PEIXOTO, A. J.; HSU, L. Controle por realimentação de saída para sistemas incertos fortemente não-lineares. Revista Controle \& Automação (SBA), v. 21, p. 69-81, 2010.

Oliveira, Peixoto e Hsu 2011 OLIVEIRA, T. R.; PEIXOTO, A. J.; HSU, L. Controle por realimentação de saída e modos deslizantes via função de chaveamento periódica aplicado ao problema de busca extremal. Revista Controle \& Automação (SBA), v. 22, p. 412-424, 2011.

Plestan e Grizzle 1999 PLESTAN, F.; GRIZZLE, J. W. Synthesis of nonlinear observers via structural analysis and numerical differentiation. In: 1999 European Control Conference. September: [s.n.], 1999.

Reger, Mai e Sira-Ramirez 2006 REGER, J.; MAI, P.; SIRA-RAMIREZ, $\mathrm{H}$. Robust algebraic state estimation of chaotic systems. In: IEEE 2006 CCA/CACSD/ISIC. Munich, Germany: [s.n.], 2006. p. 326-331.

Reger, Sira-Ramirez e Fliess 2005 REGER, J.; SIRA-RAMIREZ, H.; FLIESS, M. On non-asymptotic observation of nonliner systems. In: 44th IEEE Conference on Decision and Control. Sevilla, Spain: [s.n.], 2005.

Stephan et al. 2013 STEPHAN, R. et al. Mancais Magnéticos: mecatrônica sem atrito. Rio de janeiro: Ciência Moderna, 2013. 227 p. 
Apêndice A

Artigo: Closed-loop performance analysis of algebraic estimators 


\title{
Closed-Loop Performance Analysis of Algebraic Estimators
}

\author{
Zoraida Violeta Lopez Murgueytio • \\ Paulo Sérgio Pereira da Silva . \\ Carlos Eduardo de Brito Novaes
}

Received: 18 April 2011 / Revised: 3 April 2012 / Accepted: 26 July 2012 / Published online: 12 October 2013

(C) Brazilian Society for Automatics-SBA 2013

\begin{abstract}
The closed-loop behavior of algebraic estimators is studied in this work via the simulation of an example. The algebraic estimators can be implemented as time-varying filters that gives an estimation of derivatives of the input signal. One considers here the inverted pendulum over a car as a representant of a class of systems with state $x=\left(y^{\top}, \dot{y}^{\top}\right)^{\top}$, where $y \in \mathbb{R}^{m}$ is the output and $\dot{y} \in \mathbb{R}^{m}$ is its time derivative. For this class of systems, which includes several important mechanical systems, the estimation of the state relies on the determination of the derivative of the output. The case study that is presented in this paper indicates many interesting properties of those estimators and allows one to state many conjectures that can be considered in a future research. This work includes also a theoretical contribution that allows to compute a bound of the error of the second-order algebraic estimator. Furthermore, it is shown that all estimators that respects this bound will assure closed-loop stability in the context of the separation principle for this particular class of systems.
\end{abstract}

Keywords Algebraic estimators - Stability - Control of mechanical systems $\cdot$ Nonlinear systems $\cdot$ Separation principle.

Z. V. L. Murgueytio · P. S. Pereira da Silva ( ） · C. E. de Brito Novaes PTC, Escola Politécnica da Universidade de São Paulo, São Paulo, SP 05508-900, Brazil

e-mail: paulo@lac.usp.br

Z. V. L. Murgueytio

e-mail: zoraida_1@hotmail.com

C. E. de Brito Novaes

e-mail: carlosnov@gmail.com

\section{Introduction}

The control of nonlinear systems in observer-controller topology is an important issue in control theory (Cunha et al. 2005; Ahrens and Khalil 2009; Oliveira et al. 2010, 2011). This topology is directly related with different versions of the separation theorem for nonlinear systems (Atassi and Khalil 1999, 2000, 2001), and it can be generalized to solve problems of tracking (Peixoto et al. 2011).

The idea of replacing the traditional observers by estimations of output derivatives is an old idea of estimation theory. This idea is consistent with the theoretical result that shows that a nonlinear system with state $x$, input $u$ and output $y$ given by

$\dot{x}(t)=f(x(t), u(t))$,

$y=h(x(t))$,

is observable if and only if its state can be determined from the output and its derivatives, and the input and its derivatives. In other words, there must be an explicit formula $x=\psi\left(u, \dot{u}, \ldots, u^{(\mu)}, y, \dot{y}, \ldots, y^{(\eta)}\right)$ (Diop and Fliess 1991). Several works have showed that observers can be replaced by estimates of the derivatives of $y$ and $u$ (Plestan and Grizzle 1999; Diop et al. 2000; Diop et al. 1994; Ibrir and Diop 2004; Levant 2003).

The algebraic method of estimation of derivatives, introduced in Fliess and Sira-Ramirez (2004a,b), uses the properties of the operational calculus to build an estimator of derivatives of arbitrary order of a given signal $y(t)$. For simplicity, the reader may consider that these properties are essentially the properties of the Laplace transform (Mikusinski 1960) without any loss of understanding. Such estimator can be implemented by a linear time-varying filter (Reger et al. 2005, 2006). Recently, it was shown that such filters 
can be computer-implemented after a suitable discretization. Furthermore, the fundamental properties of those estimators are maintained for their digital implementations (Novaes and Silva 2009; Novaes 2010). Several successful applications in the control of nonlinear systems are reported in Fliess and Ramirez (2004). It is also important to stress that the algebraic estimators introduced by Fliess and Sira-Ramirez have connections with the results of Chen and Lee (1995), Dabroom and Khalil (1999) and Duncan et al. (1996).

The algebraic estimators are called fast estimators, or nonasymptotic estimators, because they are able to produce arbitrarily accurate estimations in an arbitrarily fast manner. The estimator considered in this paper is the simplest estimator of this family, which is able to estimate only the first-order derivative of the signal.

As we shall see in Sect. 3.1, this method produces an estimate of the derivative with an estimation error that grows with time. This is due to the fact that, within a time interval $[0, h]$, the signal is approximated by a polynomial of degree $k$ (the order of the estimator). This approach is good for small values of $h$, but may produce unacceptable errors for higher values of $h$. Thus, to ensure the desired accuracy of the estimate, a $h$-periodic reset may be performed.

Our theoretical contribution is to establish a formula that provides a bound for the estimation error of the second-order estimator. Furthermore, this formula is applied in order to obtain an important result of closed-loop stability for a class systems. Up to our best knowledge, both results are new in the literature. Although the results so obtained hold only for the second-order estimator and for a particular class of nonlinear systems ${ }^{1}$, we believe that the techniques developed here can be generalized for higher-order estimators, as well as for more general classes of systems. The nature of the closed-loop stability results presented in this work is close to a separation theorem (see for instance Atassi and Khalil 1999, 2000, 2001).

The interesting property that occurs is that the noise immunity of the estimation grows with the increase in $h$ while the estimation accuracy, as stated earlier, decreases with increasing $h$. This compromise between accuracy and rejection of noise is deeply discussed in Novaes (2010), and remains in the digital implementation of these estimators.

A recent work Mboup et al. (2009) has shown that the algebraic estimators can be implemented without the need to re-initialization. However, the present work deals with the version of the time-varying filters that still require $h$-periodic re-initialization.

To motivate our theoretical results, many computer simulations comparing performance and robustness of actual state feedback with feedback of the estimated state are presented. The chosen system is the popular inverted pendulum on a

\footnotetext{
${ }^{1}$ This class does not consider systems that exhibit zero dynamics.
}

cart. This system has the property that its state is of the form $x=\left(y^{\top}, \dot{y}^{\top}\right)^{\top}$ where $y$ is the output vector (see Sect. 4). In this case, the state estimation reduces to estimation of the first-order output derivative. The basic control law is a state feedback $u=F x=F_{1} y+F_{2} \dot{y}$ projected for the linearized system around the origin. This closed-loop system with state feedback will be called $S F$, and the feedback $F$ is designed in a way that the origin is locally asymptotically stable. The closed-loop system obtained by replacing $\dot{y}$ by the output of the algebraic estimator is called $S F A$. In this case, if $\widehat{\dot{y}}$ is the output of the algebraic estimator, the control law is $u=F_{1} y+F_{2} \hat{\dot{y}}$. The presented simulations regard a comparison of performance and robustness of these two control laws $S F$ and $S F A$.

We decided to restrict our comparisons only between the state feedback SF and estimated state feedback SFA via the algebraic method, avoiding the comparison with traditional asymptotic observers. In fact, no observer can be better than the perfect information of state, and we are comparing the algebraic estimator with the "perfect observer" or the "perfect estimator".

The comparisons are divided into three cases:

- Performance comparisons for several values of $h$;

- Comparisons of noise immunity for various values of $h$;

- Comparisons of robustness for various values of $h$.

The expected results of those comparisons of performance, robustness and noise immunity are that the closedloop behavior of the algebraic method should approximate the behavior of perfect state feedback as $h$ becomes small. Moreover, due to the results of Novaes (2010), it is expected that noise immunity should increase when $h$ is increased, since this is the behavior that is observed for the open-loop estimator.

The estimator implemented in this work is the simplest case of the filter described in Reger et al. (2005, 2006), that is, it is the second-order filter. It is important to point out that the research of this class of estimators has evolved since the publication of the seminal ideas of Fliess and Sira-Ramirez about algebraic estimators. Some older versions of the algebraic estimators have allowed the computation of the initial condition of a system. Then, a periodic re-initialization could provide a constant by parts state estimation. The version studied here allows a continuous state estimator, with an error that can be controlled by the reset period $h$ of such filter.

The organization of the article is described below. In Sect. 2, we introduce a brief description of the algebraic estimators, particularizing the presentation for the second-order estimator. The theoretical contribution of the paper is presented in Sect. 3. The inverted pendulum model is described in Sect. 4. Sections 5 and 6 describe the control by state feedback (SF) and feedback control of the estimated state (SFA), 
which were used in the simulations of the inverted pendulum that are presented in Sect. 7. In Sect. 7, we present the results of simulations, comparing control laws SF and SFA. In Sect. 8, we present some conclusions. Finally, in Appendix, we present the proofs of closed-loop stability that regards our separation theorems.

\section{Description of the Algebraic Method}

In this section, we briefly present the notion of algebraic estimators. For details, the reader should refer Fliess and SiraRamirez (2004a,b), Fliess and Ramirez (2004) and Reger et al. $(2005,2006)$.

\subsection{The Basic Idea of the Method}

Consider an arbitrary $C^{\infty}$ signal $y(t)$, defined on the nonnegative real axis. We know that the signal value $y(t)$ for $t>0$ can be approximated by the classical truncated Taylor series expansion:

$\tilde{y}(t)=\sum_{j=1}^{k} \frac{1}{(j-1) !} y^{(j-1)}(0) t^{(j-1)}$,

where $y^{(k)}(0)$ represents the of $k$-th order time derivative of $y(t)$ evaluated at $t=0$.

Obviously, higher is the value of $k$, smaller is the difference $y(t)-\tilde{y}(t)$, and this can be deduced from an error formula of the Taylor expansion. For instance, consider the Lagrange formula:

$R_{k}(t) \leq \frac{1}{k !} \sup _{\tau \in[0, t]}\left|y^{(k)}(\tau)\right|$.

Note that, if $y$ is analytic in an interval $[0, h]$, then $\lim _{k \rightarrow \infty} R_{k}(t)$ will be zero uniformly in $t$ for $t \in[0, h]$, at least when $h$ is smaller than the convergence radius of the Taylor series.

Notice now that the truncated Taylor series (1) can be identified with the response of a linear homogeneous system with a set of initial conditions that may be represented by the initial value $y(0)$ of the signal and also the derivatives $y^{(1)}(0), y^{(2)}(0), \ldots, y^{(k-1)}(0)$ of the signal $y(t)$, also evaluated at $t=0$. In this case, we can write:

$\tilde{y}^{(k)}(t)=0$

$\tilde{y}^{(j)}(0)=y^{(j)}(0), \quad j=0, \ldots, k-1$.

In the notation of Operational Calculus, an estimate $\tilde{y}(s)$ of the signal $y(t)$ can be represented by

$s^{k} \tilde{y}(s)-\sum_{j=1}^{k} s^{k-j} \tilde{y}^{(j-1)}(0)=0$.
The above equation may be also obtained by applying Laplace transform into Eq. (2). The k-th order derivative of Eq. (4) with respect to the operator $s$ yields the following expression:

$\frac{\mathrm{d}^{k}}{\mathrm{~d} s^{k}}\left(s^{k} \tilde{y}(s)\right)=0$

that is independent of any unknown initial conditions. Multiplying the left by $s^{-j}$, Eq. (5) for $j=k-1, \ldots, 2$, , we obtain

$s^{-j} \frac{\mathrm{d}^{k}}{\mathrm{~d} s^{k}}\left(s^{k} \tilde{y}(s)\right)=0$.

This is an important expression, which provides a triangular system of linear equations from which the time derivatives of $\tilde{y}$ of order between 1 and $k-1$ can be calculated. The idea then is to adopt these functions so obtained as approximations of the time derivative of the original signal $y(t)$, re-transforming them to time domain (6) and thereby obtaining explicit formulas for approximating time derivatives of $y(t)$. In the following section, we will study the particular case where $k=2$, showing how (6) can be solved.

\subsection{Dynamics of the Second-Order Estimator}

From now on, the actual value of the output signal will be denoted by $y(t)$, and the value of its polynomial approximation of degree 1 will be denoted by

$\tilde{y}=y(0)+y^{(1)}(0) t$.

The time-derivative estimation given by the algebraic estimator is denoted by $\widehat{\dot{y}}(t)$. The true derivative of the signal will be denoted by $\dot{y}(t)$, or by $y^{(1)}(t)$.

Consider the first-order approximation, at time $t=0$, i.e., the truncation of the Taylor series for $k=2$. Substituting $k=2$ into the Eq. (1), we get:

$\tilde{y}(t)=\sum_{j=1}^{2} \frac{1}{(j-1) !} y^{(j-1)}(0) t^{j-1}$.

Note that $\tilde{y}(t)$ satisfies the homogeneous linear timeinvariant differential equation

$\tilde{y}^{(2)}(t)=0$.

This last equation may be considered in the framework of operational calculus, as seen in Eq. (5), yielding

$\frac{\mathrm{d}^{2}}{\mathrm{~d} s^{2}}\left(s^{2} \tilde{y}(s)\right)=0$.

Substituting values $k=2$ and $j=1$, in the Eq. (6), we obtain:

$s^{-1} \frac{\mathrm{d}^{2}}{\mathrm{~d} s^{2}}\left[s^{2} \tilde{y}(s)\right]=0$. 
From (8), we have

$s^{-1} \frac{\mathrm{d}}{\mathrm{d} s}\left[\frac{\mathrm{d}}{\mathrm{d} s}\left(s^{2} \tilde{y}(s)\right)\right]=0$

and so:

$2 s^{-1} \tilde{y}(s)+4 \frac{\mathrm{d} \tilde{y}(s)}{\mathrm{d} s}+s \frac{\mathrm{d}^{2} \tilde{y}(s)}{\mathrm{d} s^{2}}=0$.

Using the basic properties of the Laplace transform, Eq. (9), when transformed back to time domain reads:

$2\left(\int_{0}^{t} \tilde{y}(\sigma) \mathrm{d} \sigma\right)-4 t \tilde{y}(t)+\frac{\mathrm{d}}{\mathrm{d} t}\left[t^{2} \tilde{y}(t)\right]=0$.

Note that

$\frac{\mathrm{d}}{\mathrm{d} t}\left[t^{2} \tilde{y}(t)\right]=2 t \tilde{y}(t)+t^{2} \tilde{y}^{(1)}(t)$.

Returning to Eq. (10) and substituting the expression above, we obtain:

$\left.2\left(\int_{0}^{t} \tilde{y}(\sigma) \mathrm{d} \sigma\right)-2 t \tilde{y}(t)+t^{2} \tilde{y}^{(1)}(t)\right)=0$.

Solving for $\tilde{y}^{(1)}$, we can write:

$\tilde{y}^{(1)}(t)=\frac{1}{t^{2}}\left[-2 \int_{0}^{t} \tilde{y}(\sigma) \mathrm{d} \sigma+2 t \tilde{y}(t)\right]$.

The idea of implementing the estimator is to replace, in the above equation, $\tilde{y}$ for $y$, obtaining the following approximate formula:

$\widehat{\dot{y}}(t)=\frac{1}{t^{2}}\left[-2 \int_{0}^{t} y(\sigma) \mathrm{d} \sigma+2 t y(t)\right]$.

During an interval $[0, \epsilon]$, for $\epsilon$ very small, we have a numerical indetermination in the value of the calculated derivative $\dot{y}(t)$. The value of $\epsilon$ is chosen for reasons of computational precision of the division by a number $t^{2}$ that is very small. This motivates the introduction of two parallel estimators that are also subject to $h$-periodically reset, but at different instants.

\subsection{Parallel Estimation}

In the next section, we will show that the algebraic estimator has good accuracy only for a small time interval. It is then necessary to restart the calculations for each sufficiently small period of time to ensure the quality of the estimation. Define then a reset interval of length $h$ seconds, meaning that the estimation is reset when $t_{k}=k h, k \in N$. With the purpose of increasing the accuracy of the algebraic estimation, this idea was introduced in (Fliess and Sira-Ramirez 2004a,b). This technique was called parallel estimation, and it proposes to implement two estimators that works simultaneously. The first estimator is re-initialized at the instants of time $t_{k}=h k$ for $k=0,1,2, \ldots$, and the second estimator is re-initialized in $t_{k}=h k+h / 2$. The output $\widehat{\dot{y}}_{1}(t)$ of the first estimator is taken while $t \in\left[\frac{h(2 k+1)}{2},(k+1) h\right)$, and the output $\widehat{\dot{y}}_{2}(t)$ of the second estimator is taken in the intervals $t \in\left[h k, \frac{h(2 k+1)}{2}\right)$. The output value of each estimator in parallel can be calculated by the same formula (12) given below. It is important to point out that in this formula, the values of $t_{k}$ (the reset instants) of each estimator are different from each other.

$\widehat{\dot{y}}(t)=\frac{\left[-2 \int_{t_{k}}^{t} y(\sigma) \mathrm{d} \sigma+2\left(t-t_{k}\right) y(t)\right]}{\left(t-t_{k}\right)^{2}}$.

In the above equation, $\widehat{\dot{y}}(t)$ denotes the estimated $\dot{y}(t)$. Thus, accurate results of the estimation can be obtained at any instant time $t \geq 0$, except for the initial moments $t \in[0, \epsilon)$, for which both estimators produce invalid estimations ${ }^{2}$.

\section{Properties of the Second-Order Estimator}

In this section, we present the theoretical contribution of this article, that is, a formula that gives a bound of the estimation error and the proof of the closed-loop stability for a class of systems.

\subsection{Accuracy Analysis of Second-Order Estimators}

The error analysis for the formula (11) is now performed. We consider $t \in[\epsilon, h]$ where $\epsilon>0$ is sufficiently large to avoid the singularity of the division by $t^{2}$, and $h$ is the chosen re-initialisation period of the algebraic estimator.

Let $y:[0, h] \rightarrow \mathbb{R}$ a signal of class $C^{2}$. Let

$\tilde{y}(t)=y(0)+y^{(1)}(0) t$,

be the first-order Taylor approximation of $y(t)$. In this analysis, $M(t)$ will be the maximum value that $y^{(2)}$ assumes in the compact interval $[0, t]$. In other words,

$M(t)=\sup _{\tau \in[0, t]}\left|y^{(2)}(\tau)\right|$.

Recall that

$y(t)=\tilde{y}(t)+R_{2}(t)$,

where $R_{2}(t)$ is the Taylor remainder. The Lagrange remainder formula gives (vide Lima 1981, p. 91):

$\left|R_{2}(t)\right| \leq M|t|^{2} / 2$.

\footnotetext{
2 Remember that $\epsilon$ is the size of the interval in which the estimators produce bad results after initialization ( $\epsilon$ was defined in Sect. 2.2).
} 
The time derivation of (13) in both sides gives

$$
y^{(1)}(t)=\tilde{y}^{(1)}(t)+R_{2}^{(1)}(t)
$$

As $\tilde{y}^{(1)}(t)=y^{(1)}(0)$, it follows that

$$
R_{2}^{(1)}(t)=y^{(1)}(t)-y^{(1)}(0)
$$

By the mean value theorem (vide Lima 1981, p. 89), there $\xi \in[0, t]$ such that $y^{(1)}(t)-y^{(1)}(0)=y^{(2)}(\xi)(t-0)$. therefore

$$
\left|R_{2}^{(1)}(t)\right| \leq M|t|
$$

Substituting $y=\tilde{y}+R_{2}$ em (11), we obtain

$$
\begin{aligned}
\widehat{\dot{y}}(t)= & \frac{1}{t^{2}}\left[-2 \int_{0}^{t} \tilde{y}(\sigma) \mathrm{d} \sigma+2 t \tilde{y}(t)\right] \\
& +\frac{1}{t^{2}}\left[-2 \int_{0}^{t} R_{2}(\sigma) \mathrm{d} \sigma+2 t R_{2}(t)\right] .
\end{aligned}
$$

Recall from the previous section that $\tilde{y}$ is always a solution of $^{3}$

$\tilde{y}^{(1)}(t)=\frac{1}{t^{2}}\left[-2 \int_{0}^{t} \tilde{y}(\sigma) \mathrm{d} \sigma+2 t \tilde{y}(t)\right]$.

Define

$\Delta(t)=\frac{1}{t^{2}}\left[-2 \int_{0}^{t} R_{2}(\sigma) \mathrm{d} \sigma+2 t R_{2}(t)\right]$.

then, (11) provides

$\widehat{\dot{y}}(t)=\tilde{y}^{(1)}(t)+\Delta(t)=\tilde{y}^{(1)}(0)+\Delta(t)$.

Therefore, the error of the derivative estimation will be

$$
\begin{aligned}
e(t) & =\widehat{\dot{y}}(t)-y^{(1)}(t), \\
& =\tilde{y}^{(1)}(0)+\Delta(t)-\tilde{y}^{(1)}(0)-R_{2}^{(1)}(t), \\
& =\Delta(t)-R_{2}^{(1)}(t) .
\end{aligned}
$$

As we already have an upper bound for $R_{2}^{(1)}(t)$, it remains to provide an upper bound for $\Delta(t)$. To do this, note that, using (15), the triangle inequality and the fact that $\left|\int_{0}^{t} f(\sigma) \mathrm{d} \sigma\right| \leq \int_{0}^{t}|f(\sigma)| \mathrm{d} \sigma$ for every continuous function $f$, it follows that:

\footnotetext{
3 This is equivalent to saying that the estimate is exact for a polynomial signal of degree 1 .
}

$$
\begin{aligned}
|\Delta(t)| & =\left|\frac{1}{t^{2}}\left[-2 \int_{0}^{t} R_{2}(\sigma) \mathrm{d} \sigma+2 t R_{2}(t)\right]\right|, \\
& \leq \frac{1}{t^{2}}\left\{\left|2 \int_{0}^{t} M \frac{\sigma^{2}}{2} \mathrm{~d} \sigma\right|+M\left|t^{3}\right|\right\}, \\
& \leq \frac{4}{3}|t| M .
\end{aligned}
$$

Thus, by (14), an upper bound for the estimation error inside the interval $[0, t]$ will be:

$$
\sup _{t \in[0, t]}|e(t)| \leq \frac{7}{3}|t| \sup _{t \in[0, t]}\left|y^{(2)}(t)\right| .
$$

In particular, if the estimators are $h$-periodically reset, an upper bound for the estimation error of (12) will be

$$
\sup _{t \in[0, h]}|e(t)| \leq \frac{7}{3}|h| \sup _{t \in[0, h]}\left|y^{(2)}(t)\right| \text {. }
$$

We see that the maximum error is proportional to the maximum of the second derivative of the signal $y(t)$ and is also proportional to the reset period $h$ of the algebraic estimator.

\subsection{Closed-Loop Stability}

In this section, we study closed-loop stability of a class of systems, introducing a separation-like theorem. The basic difference of our results with respect to a classical separation theorem is that, although the dynamics of the closed-loop system is altered by the inclusion of the estimator dynamics, due to the special nature of the algebraic estimators, the proof of closed-loop stability only needs to consider the original system dynamics, which is modified by the presence of the estimation error (19). In other words, the order of the closedloop system studied in the proof of our stability theorem is the same order of the open-loop system. This fact does not occur in most of the proofs of different versions of separation theorem (see for instance Atassi and Khalil 1999, 2000, 2001). Although the class of systems is limited, we believe that the techniques developed here can be extended to more general classes of systems.

Let $y(t) \in \mathbb{R}^{m}$ and $u(t) \in \mathbb{R}^{l}$. Consider an affine system of class $C^{1}$ with input $u(t)$ and output $y(t)$, given by:

$$
\begin{aligned}
\dot{y}(t) & =y^{(1)}(t), \\
\dot{y}^{(1)}(t)= & F\left(t, y(t), y^{(1)}(t)\right) \\
& +G\left(t, y(t), y^{(1)}(t)\right) u(t) .
\end{aligned}
$$

Let

$$
\begin{aligned}
x(t) & =\left(y(t)^{\top}, y^{(1)}(t)^{\top}\right) \in \mathbb{R}^{n}, \\
f(t, x(t)) & =\left(y^{(1)}(t)^{\top}, F(t, x(t))^{\top}\right)^{\top},
\end{aligned}
$$




$$
g(t, x(t))=\left(0, G(t, x(t))^{\top}\right)^{\top},
$$

$h(t, x(t))=y(t)$.

Note that, the class of studied systems is a subclass of time-varying affine systems, given by:

$\dot{x}(t)=f(t, x(t))+g(t, x(t)) u(t)$,

$y(t)=h(x(t))$,

$x\left(t_{0}\right)=x_{0}$,

with $f, g, h$ given by (21).

Assume that the origin is an equilibrium point of system (20) for null input. In other words, for the system rewritten as (22), we get

$f(t, 0)=0, \quad \forall t \geq t_{0}$.

As usual in nonlinear systems theory, there is no loss of generality in studying only the stability of the origin. The stability around other points of equilibrium with $y \neq 0$ is completely analogous. It is important to point out that for the studied class of systems, $\dot{x}=0$ implies $y^{(1)}=0$.

In different versions of the separation principle that we will prove, we will assume that a state feedback

$u(t)=\alpha\left(t, y(t), y^{(1)}(t)\right)$

asymptotically stabilizes the origin of the system (20). Sometimes it will be more convenient to use the compact notation of Eq. (22). In this case, being consistent with the notation of Eq. (21a), we will use the notation

$\alpha(t, x) \doteq \alpha\left(t, y, y^{(1)}\right)$

where $\doteq$ denotes "equal by definition". In Theorems 1 and 2, we assume that the feedback of the estimated state $\hat{x}$ defined by

$\hat{x}=\left(y^{\top}, \hat{\dot{y}}^{\top}\right)^{\top}$,

and applied in the system (20). In other words, the following control law is considered:

$u(t)=\alpha(t, y(t), \hat{\dot{y}}(t))$

where $\hat{\dot{y}}(t)$ is the output of the algebraic estimator (12) with $h$-periodic reset and parallel estimation. Hence, sometimes it will be convenient to denote this control law in the compact form below:

$u=\alpha(t, \hat{x})$.

Before stating the first stability theorem, we present some important assumptions that are considered by this result. Assume that

Hypothesis H1 The following assumptions hold for system (20) and the control law (26) of class $C^{1}$ :
- $F(t, 0,0)=0, \forall t \geq t_{0}$.

- There exists $\gamma$ positive such $\left\|G\left(t, y, y^{(1)}\right)\right\| \leq \gamma$, for all $y, y^{(1)}$ belonging to $\mathbb{R}^{m}$ and for all $t \geq t_{0}$.

- Let $x$ defined by (21a) and let

$$
P(t, x)=F(t, x)+G(t, x) \alpha(t, x) .
$$

Assume that $\frac{\partial P}{\partial x}$ is uniformly bounded. In particular, as $P(t, 0)=0$ for all $t$, for all ${ }^{4} \delta>0$ such that $\|P(t, x)\| \leq$ $\delta\|x\|$.

- $\alpha(t, 0,0)=0, \forall t \geq t_{0}$.

- The map $\alpha$ is (globally) Lipschitz with respect to the third variable, that is, exists $K>0$ such that

$$
\begin{aligned}
& \| \alpha\left(t, y, y^{(1)}\right)-\alpha\left(t, y, \hat{y}^{(1)}\|\leq K\| y^{(1)}-\hat{y}^{(1)} \|,\right. \\
& \forall y, y^{(1)}, \hat{y}^{(1)} \in \mathbb{R}^{l} \text { e } \forall t \geq t_{0}
\end{aligned}
$$

Hypothesis H2 The origin of the closed-loop system with feedback (23) is globally exponentially stable. ${ }^{5}$

The following theorem presents the first version of the separation principle for this class of systems along with the parallel estimators of Sect. 2.3.

Theorem 1 Assume that we apply the control law (26) to system (20), where $\alpha(\cdot)$ obeys both Hypothesis $H 1$ and $H 2$, and $\hat{\dot{y}}(t)$ is the output of the algebraic estimator (12), with $h$-periodic reset and parallel estimation. Then, there exists a sufficiently small h such that the origin of the system (20) in closed loop with the control law (26) is globally exponentially stable.

Proof See Appendix.

We now present the hypothesis of the second stability theorem, which replaces the Hypothesis $\mathrm{H} 2$.

Hypothesis H3 Consider $x(t), f(t, x), g(t, x)$ and $h(t, x)$ as defined in (21). Let $\alpha$ the control law (23). Consider the system (20) in closed loop with (23) rewritten as

$$
\begin{aligned}
\dot{x}(t) & =f(t, x(t))+g(t, x(t)) \alpha(t, x(t)), \\
y(t) & =h(x(t)), \\
x\left(t_{0}\right) & =x_{0} .
\end{aligned}
$$

Let $n=2 m$ and assume there exists a function $V$ : $\left[t_{0}, \infty\right] \times \mathbb{R}^{n} \rightarrow \mathbb{R}$ of class $C^{1}$ and functions $W_{i}: \mathbb{R}^{n} \mapsto$ $\mathbb{R}, i=1,2,3$ such that:

(i) $W_{i}$ is continuous and positive definite around the origin, $i=1,2,3$, with $W_{1}$ radially unbounded.

(ii) $W_{1}(x) \leq V(t, x) \leq W_{2}(x), \forall t \geq t_{0}$ e $x \in \mathbb{R}^{n}$.

(iii) $\dot{V}(t, x)=\frac{\partial V}{\partial t}+\frac{\partial \bar{V}}{\partial x} \theta(t, x) \leq-W_{3}$, onde $\theta=f+g \alpha$.

\footnotetext{
${ }^{4}$ The existence of $\delta$ is guaranteed by the mean value inequality (Lima 1981).

${ }^{5}$ Refer to Definition 3.5 of Khalil (1996) for the definition of global exponential stability.
} 
(iv) There exists $\epsilon>0$ small enough such that the map $W_{4}(x)$ defined by

$$
W_{4}(x)=W_{3}(x)-\epsilon\left\|\frac{\partial V}{\partial x}\right\|\|x\|,
$$

is positive definite around the origin for $x \in \mathbb{R}^{n}$.

Remark From Theorem 3.8 and Corollary 3.3 of Khalil (1996), it follows from (i), (ii), (iii) that the closed-loop system is globally uniformly asymptotically stable ${ }^{6}$. This means that these assumptions are "natural" in the context of global asymptotic stability. However, the assumption (iv) is a technical hypothesis that might be eliminated by a converse theorem (see for example Appendix A.6 of Khalil 1996).

Theorem 2 Assume that we apply to the system (20) the control law (26). Assume that the Hypothesis HI and H3 holds for the map $\alpha(\cdot)$. Then, there exists a sufficiently small $h$ such that the origin of the system (20) in closed loop with the control law (26) is globally uniformly asymptotically stable.

Proof See Appendix.

\section{Inverted Pendulum Model}

The system that is considered for illustrating our algebraic estimator is the inverted pendulum on a car. The Eq. (30) below describes the inverted pendulum shown in Fig. 1. This model can be easily obtained using Newtonian or Lagrangian mechanics (Craig 1982).

In this figure, $M$ is the mass of the cart, $m$ is the mass of the pendulum rod, which considered to be rigid, $l$ is the length of the pendulum rod, $g$ is the acceleration of gravity, $r$ is the position of the car and $\theta$ is the rod angle with respect to the vertical direction. The output $y$ of the system is the column vector $(r, \theta)^{\top}$, and the state vector is $x=\left(y^{\top}, \dot{y}^{\top}\right)^{\top}$. Let us assume that the input $u(t)$ of the system is the force $f(t)$ that is applied in the cart, and there is no torque action on the rigid rod of length $l$. Note that $x=\left(x_{1}, x_{2}, x_{3}, x_{4}\right)^{\top}$ where $x_{1}=r, x_{2}=\theta, x_{3}=\dot{r}$ e $x_{4}=\dot{\theta}$.

The system model is then given by

$$
\dot{x}=\left(\begin{array}{c}
x_{3}(t) \\
x_{4}(t) \\
u \quad+\frac{m l x_{4}^{2} \operatorname{sen}\left(x_{2}\right)}{M+m-m \cos ^{2}\left(x_{2}\right)} \\
\frac{u g \operatorname{sen}\left(x_{2}\right) \cos \left(x_{2}\right)}{M+m-m \cos ^{2}\left(x_{2}\right)} \\
\frac{u m l \cos \left(x_{2}\right)}{\left(M+m-m \cos ^{2}\left(x^{2}\right) m g l \operatorname{sen}\left(x_{2}\right)\right.}-\frac{u l^{2}-m^{2} l^{2} \cos ^{2}\left(x_{2}\right)}{(M+m) m l^{2}-m^{2} l^{2} \cos ^{2}\left(x_{2}\right)} \\
-\frac{m^{2} l^{2} x_{4}^{2} \operatorname{sen}\left(x_{2}\right) \cos \left(x_{2}\right)}{(M+m) m l^{2}-m^{2} l^{2} \cos ^{2}\left(x_{2}\right)}
\end{array}\right) .
$$

\footnotetext{
${ }^{6}$ Refer to Definition 3.2 and Lemma 3.3 of Khalil (1996) for definition of overall asymptotic uniform stability.
}

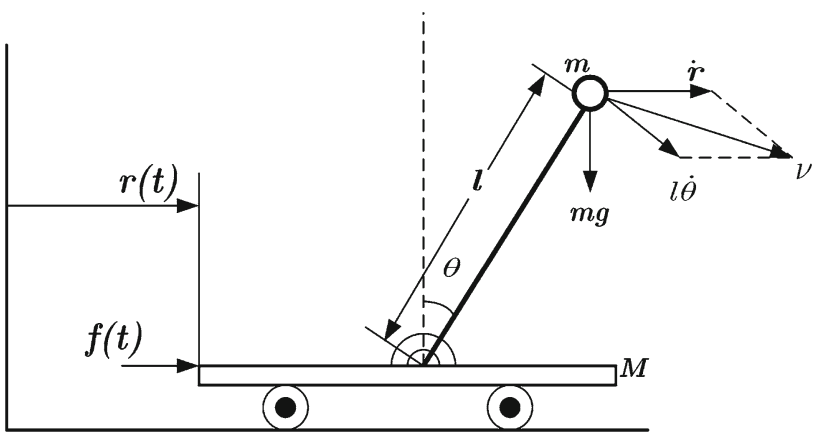

Fig. 1 Inverted pendulum on a cart

\section{Control by State Feedback (SF)}

Consider the system shown in Fig. 1. We desire to maintain the inverted pendulum in the upright position $x_{2}=0$. The tilted pendulum can be brought back to the vertical position by the application of a convenient control law. Simultaneously, we want to bring the basis for the reference position $x_{1}=0$.

For this, we have designed a control system for the linearized model of the inverted pendulum around the origin. This linearized model is controllable, and thus, we can impose poles by state feedback (Chen 1999), of the form:

$u=-F x$,

where $x \in R^{4}$ is the state vector and $F$ is the feedback gain matrix. Due to the classical result called indirect method of Lyapunov (see Khalil 1996), the origin of the closed-loop system with this control law will be locally asymptotically stable.

The following values of the physical constants of the system were adopted: $M=2 ; m=1 ; l=0.5 ; g=9.81$.

The closed-loop poles are allocated in a way that the dominant poles are given by $s_{1,2}=-2 \pm j 2 \sqrt{3}$, corresponding to a damping factor $\xi=0.5$. The two remaining poles are far enough apart so that their effect is negligible. Where chosen poles $s_{3,4}=-10$. Calculating the resultant feedback according to Chen (1999), we get: $F=[163.0989,298.1504,73.3945,60.6972]$. Seja $F=$ $\left[\begin{array}{ll}F_{1} & F_{2}\end{array}\right]$ where $F_{1}=[163.0989,298.1504]$ and $F_{2}=$ [73.3945, 60.6972]. Note that the control law is then given by:

$u=F_{1} y+F_{2} \dot{y}$.

Recall that the closed-loop system of Fig. 2 will be called $S F$. 


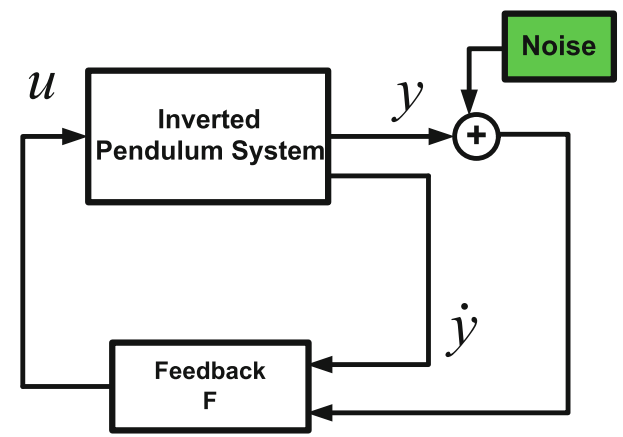

Fig. 2 State feedback control (SF)

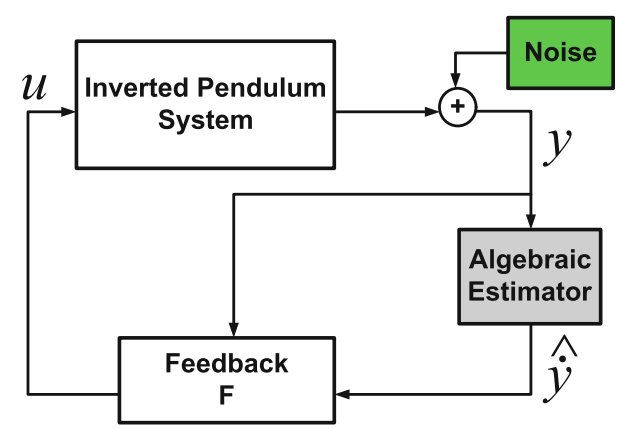

Fig. 3 State feedback of the algebraic estimate of the state (SFA)

\section{Control by Feedback of the Estimated State (SFA)}

Recall that the state $x$ in the system is of the form $x=\left(\begin{array}{l}y \\ \dot{y}\end{array}\right)$. In this subsection, we consider the second-order algebraic estimator (12), with $h$-periodically reset, and that is implemented along with the policy of parallel estimation described in Sect. 2.3. Let $\widehat{\dot{y}}$ be the output of the algebraic estimator that is implemented in this way. Consider the control law [obtained by replacing $\dot{y}$ by $\widehat{\dot{y}}$ in (31)]:

$u=F_{1} y+F_{2} \widehat{\dot{y}}$.

The closed-loop system of Fig. 3 will be called SFA.

Note that the noise shown in Fig. 3 will be amplified by algebraic estimator, while the noise shown in Fig. 2 only influences $y$. In the case of SF, the speed $\dot{y}$ is a not signal that is corrupted by noise, while for the SFA, the algebraic estimation $\widehat{\dot{y}}$ will be corrupted by noise. This will certainly provide an honest comparison, perhaps too pessimistic, because in practical applications, if the speed sensors are present, they will also provide measurements that are corrupted by noise.

\section{Comparison Between SF and SFA}

This section provides comparisons between the state feedback (SF) and the feedback of the algebraic estimation (SFA). In all simulations, the following initial condition $x_{0}=x\left(t_{0}\right)$ will be considered:

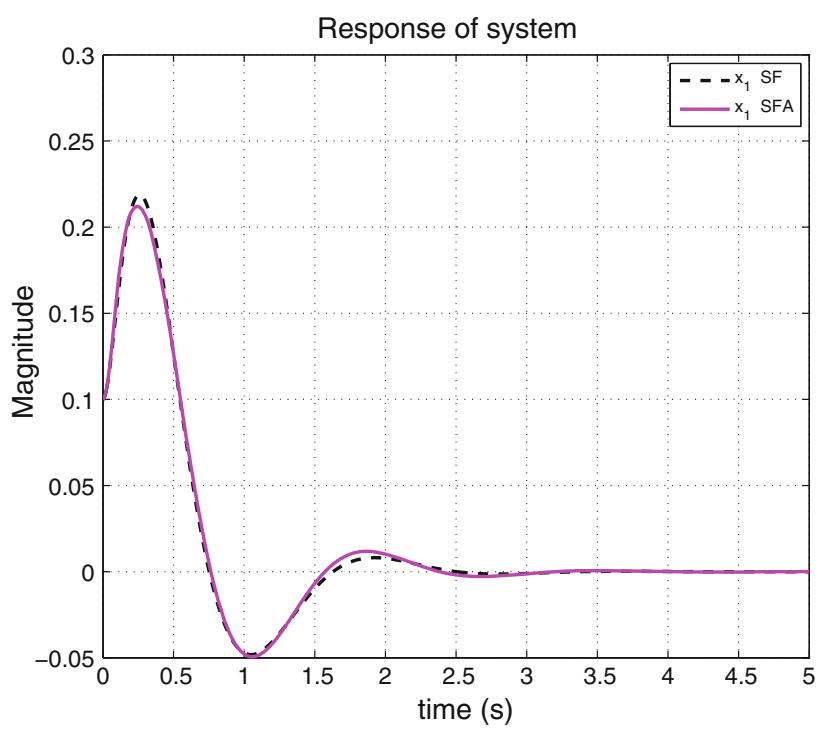

Fig. 4 Comparison of positions for $h=0.1$ without noise

$x_{0}=\left(\begin{array}{c}0.1 \\ 0.1 \\ 0 \\ 0\end{array}\right)$.

For the sake of space, we have chosen to display only the most relevant results, especially when other variables, which are not shown here, have exhibited similar qualitative results. The interested reader can refer to Murgueytio (2011), where all variables are displayed, including the output of the estimator, as well as other additional details that are not discussed here. In all simulations in the presence of noise, we use the uniform random number of Matlab/Simulink ${ }^{\circledR}$. For providing an honest comparison of RE and REA, the same seed of the random number generation was used for repeating exactly the same noise that we have applied in both cases.

\subsection{Comparing SF and SFA for Various Values of the Reset} Period $h$

To carry out the relevant tests comparing SF and SFA, we use different values for the reset period. They are $h=1 \times 10^{-6}$, $h=0.01$ and $h=0.1$ for the simulations without noise and $h=0.1$ and $h=0.05$ for simulations with noise.

Figures 4 and 6 show the behavior of the pendulum position when $h=0.1$. Figures 5 and 7 show the differences of positions between SF and SFA when $h=0.1$. It can be observed that the SFA in this case is very close to the pure state feedback SF. For the case of $h=0.01$, the Figs. 8 and 9 show even smaller position differences.

Taking a very small reset period $h$ (for instance $h=10^{-6}$ ), the performance of the closed-loop system will not improve. On the contrary, very bad results are obtained due to numeri- 


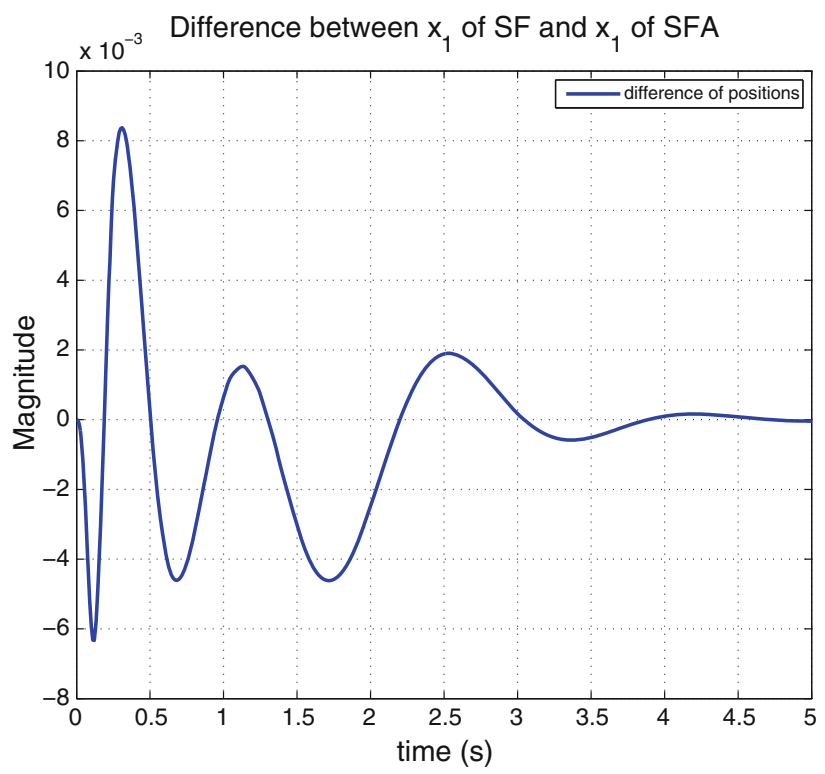

Fig. 5 Difference of positions between SF and SFA for $h=0.1$ without noise

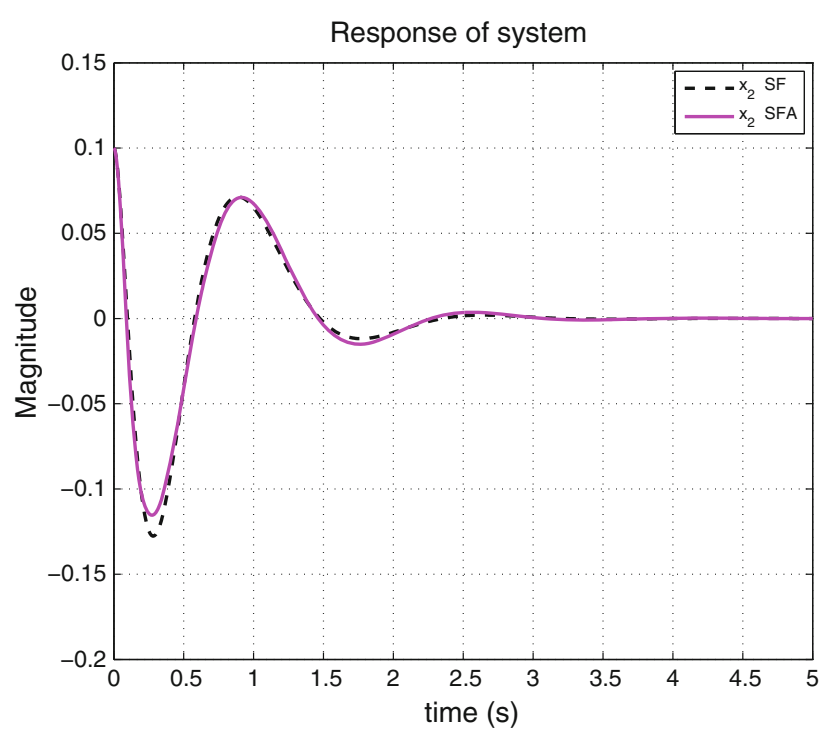

Fig. 6 Comparison of angular positions for $h=0.1$ without noise

cal problems. In fact, the computation of (12) is always close to a division by zero [see (12)]. This fact can be observed in Figs. 10 and 11 .

\subsubsection{Comparison Between SF and SFA with Measurement Noise}

Now, we repeat the previous simulations, but now applying an additive noise in the system output. The applied noise was generated by MATLAB and recorded in a variable. Recall that both simulations of the SF and the SFA use exactly the

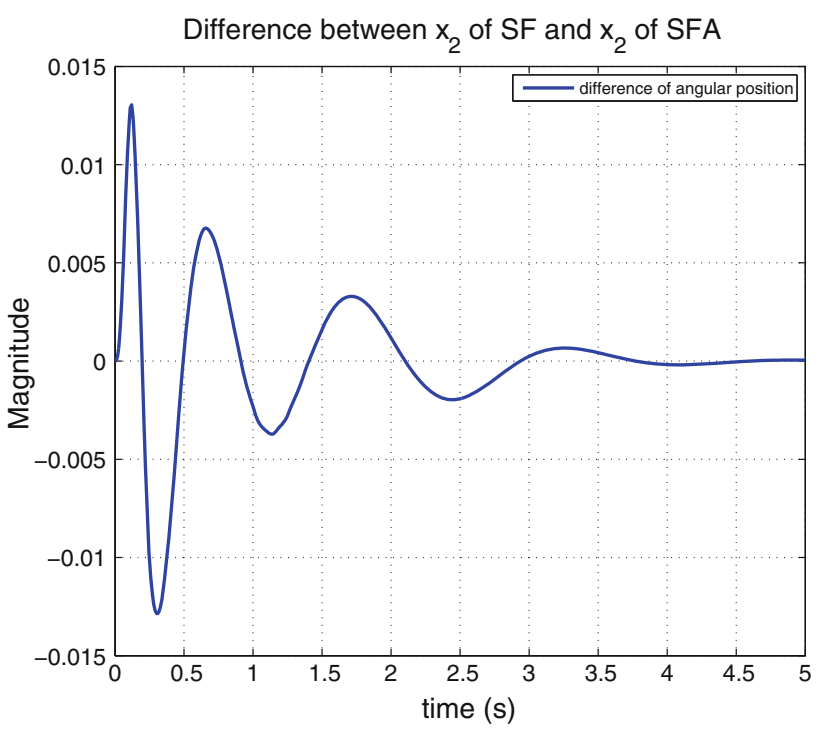

Fig. 7 Difference of angular position between SF and SFA for $h=0.1$ without noise

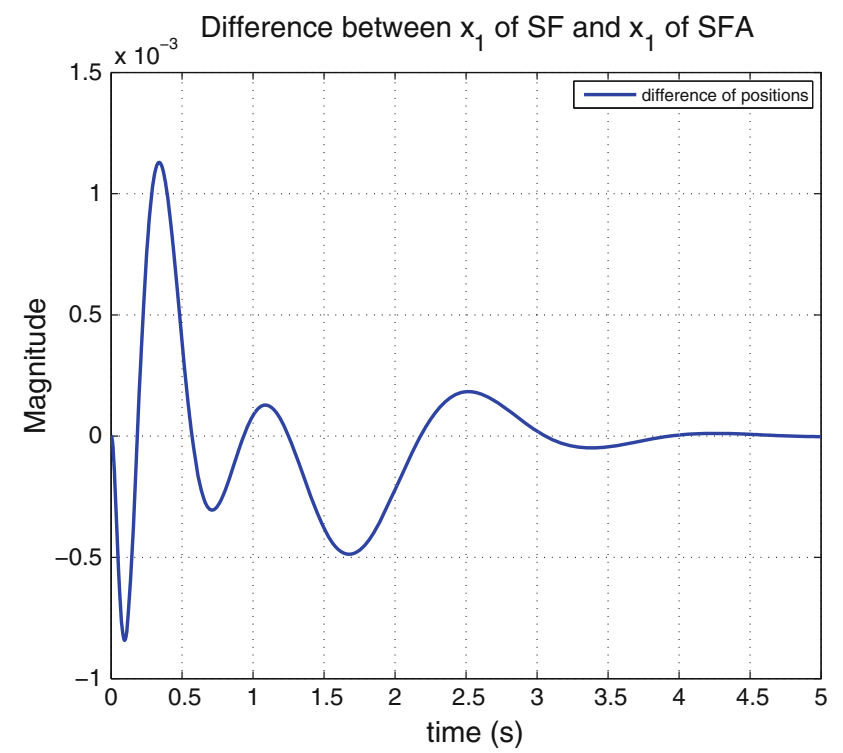

Fig. 8 Difference of positions between SF SFA for $h=0.01$ without noise

same applied noise, assuring so that the comparison is really honest.

Figures 12 and 14 show the behavior of the position of the mobile base (cart) and the angular position of the pendulum rod, under the influence of noise for $h=0.1$. Figures 13 and 15 show the differences between SF and SFA. It can be said that even in the presence of noise, the actual states $x_{1}$ e $x_{2}$ of SF and SFA are very close to each other.

For $h=0.05$, system performance is much more affected by noise, a behavior that was already found in the study of the open-loop estimator. The difference between the states of SFA and SF is greater than the ones obtained for $h=0.1$, as 


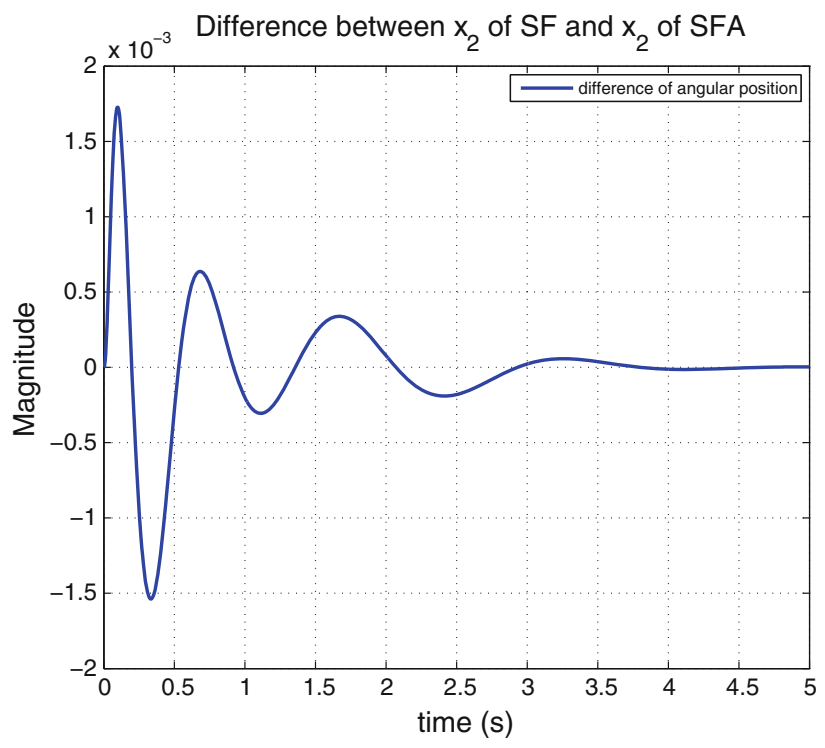

Fig. 9 Difference of angular positions beteween SF ans SFA for $h=$ 0.01 without noise

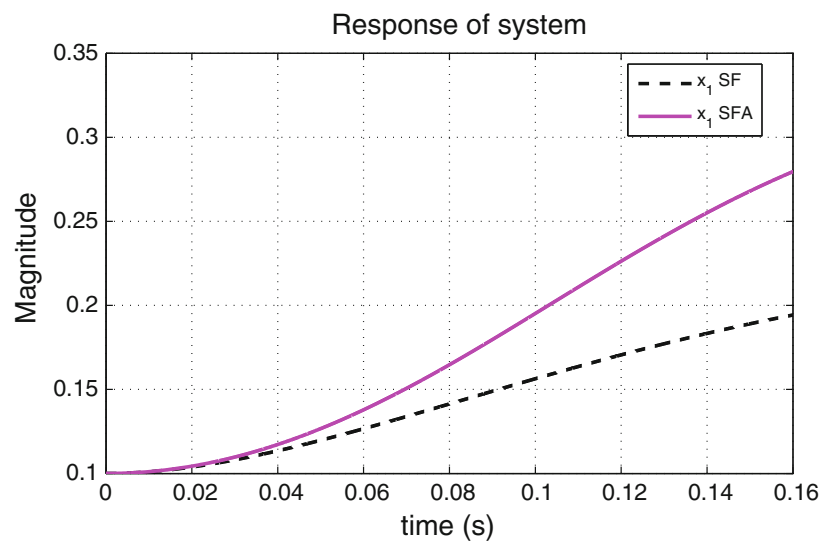

Fig. 10 Comparison of position between SF and SFA with $h=$ 0.000001 without noise

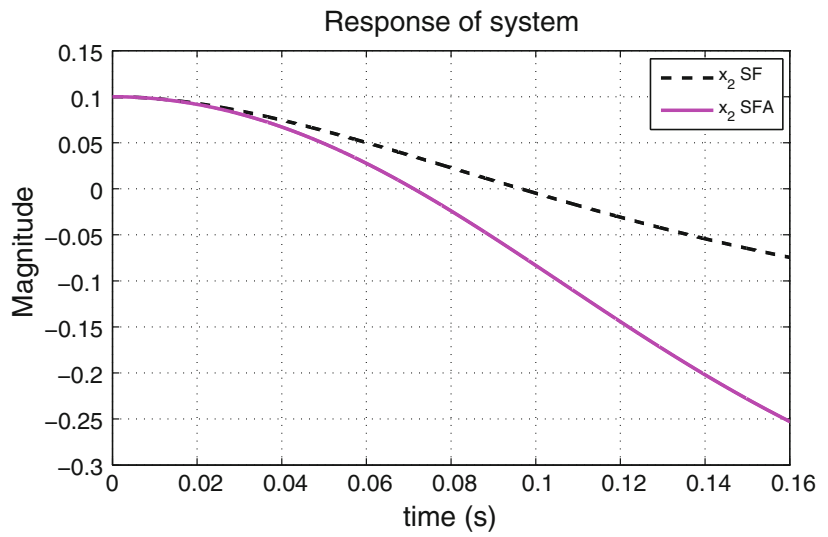

Fig. 11 Comparison of angular position between SF and SFA with $h=0.000001$ without noise

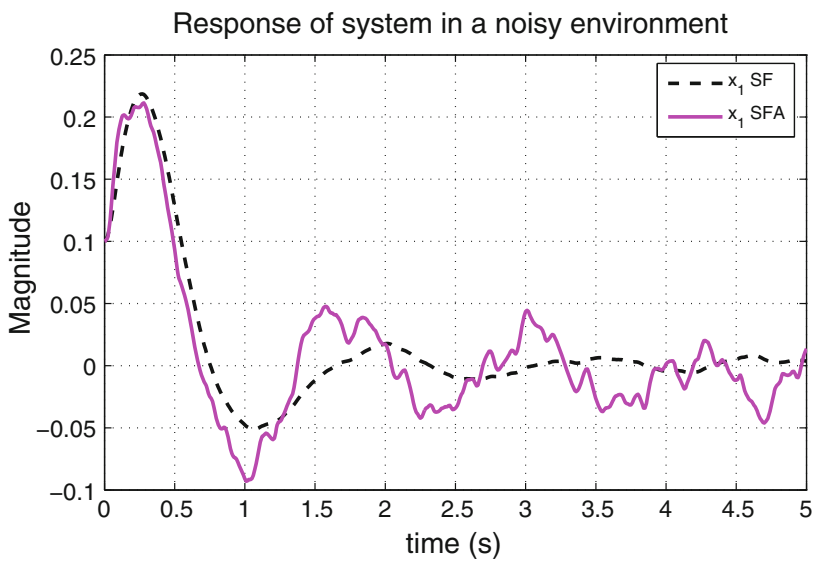

Fig. 12 Comparison of position between SF and SFA with $h=0.1$ with noise

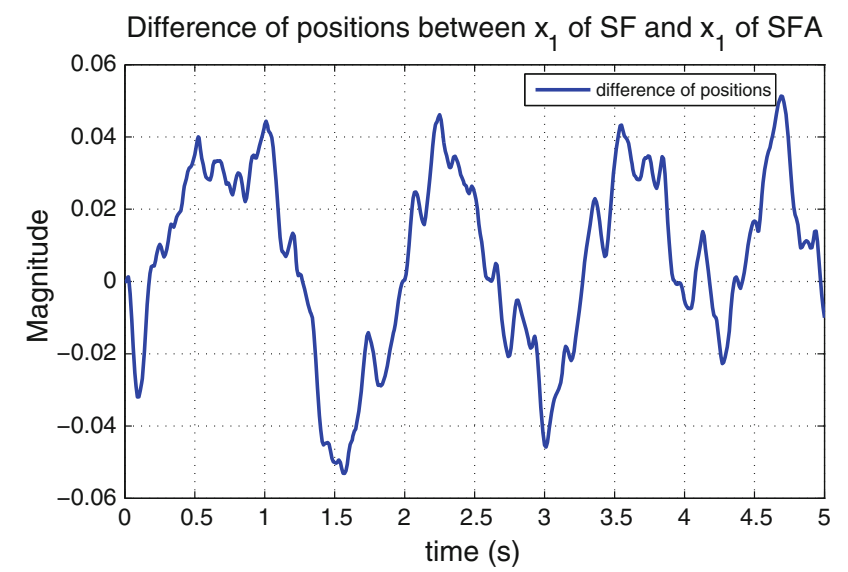

Fig. 13 Difference of positions between SF and SFA for $h=0.1$ with noise

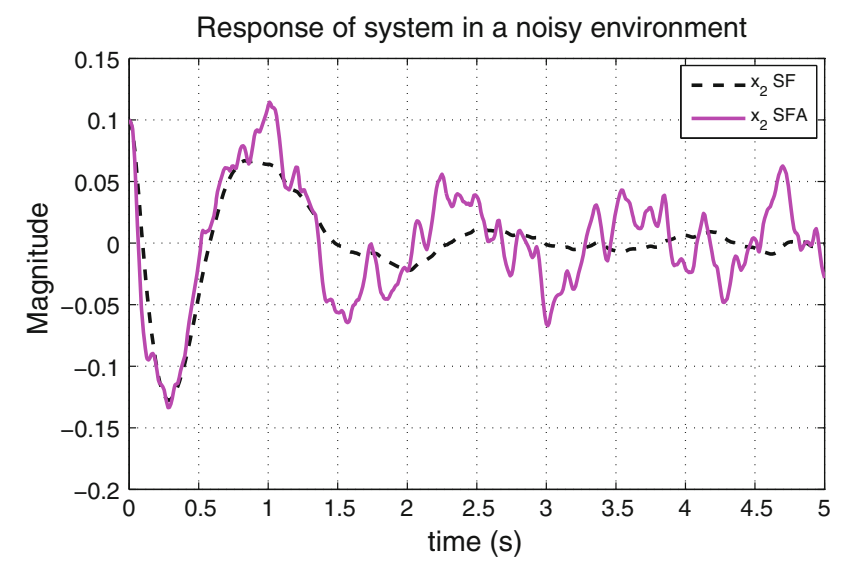

Fig. 14 Angular positions with $h=0.1$ and with noise

shown in Fig. 16. Other figures that are shown in Murgueytio (2011) allow to conclude that the estimation of $\dot{y}$ is indeed much more corrupted by noise, especially for small values of $h$. The behavior of the output in closed loop is less sensitive 


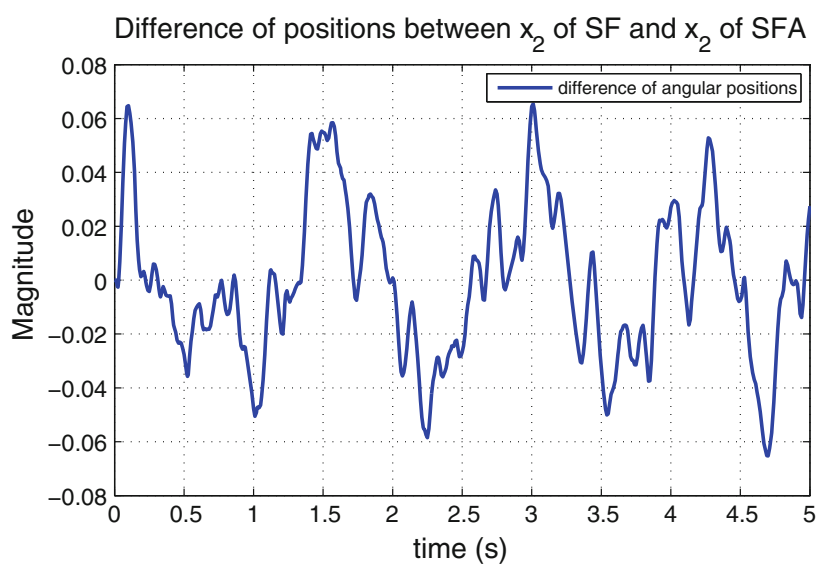

Fig. 15 Difference of angular positions between SF and SFA for $h=$ 0.1 with noise

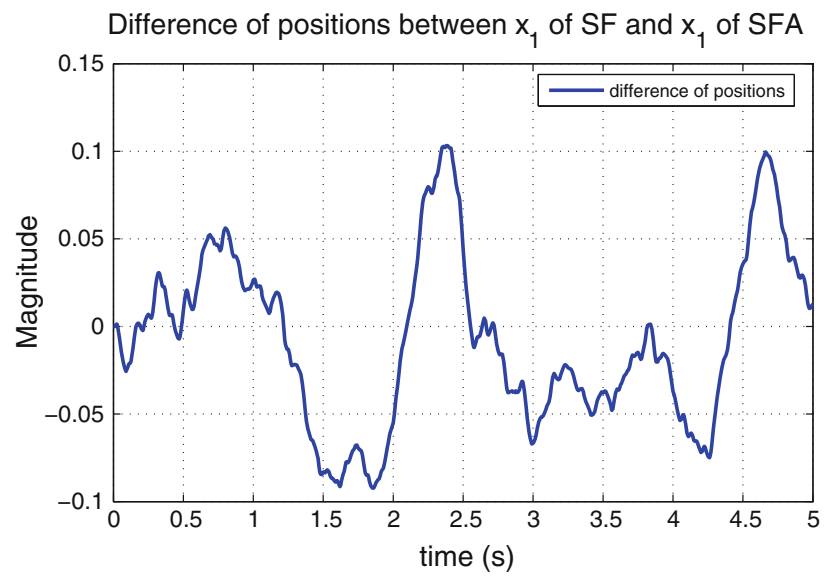

Fig. 16 Difference of positions between SF and SFA for $h=0.05$ with noise

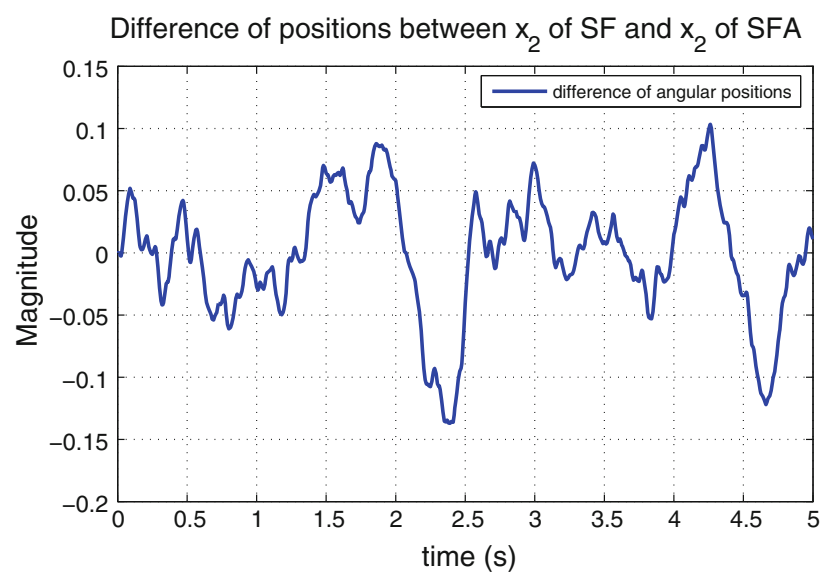

Fig. 17 Difference of positions between SF and SFA for $h=0.05$ with noise

to noise than the estimation itself. This can be explained by the fact that the mechanical system behaves as a low-pass filter (Fig. 17).

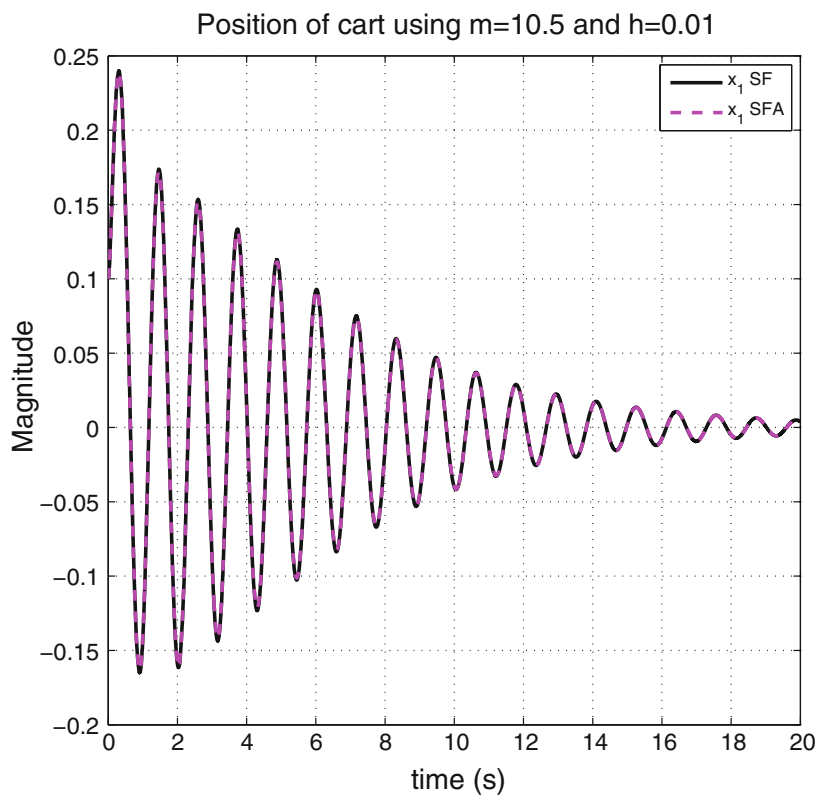

Fig. 18 Comparison of positions for SF and SFA for $m=10.5$ and $h=0.01$

\subsection{Parametric Robustness Analysis}

The parameter chosen to be modified is $m$ (mass of the pendulum rod of Fig. 1). In the inverted pendulum system, we changed only the value of the mass $m$, letting it take larger values than the nominal one, denoted by $m$, but using the same control law. We have got a range of values $\left[m, m^{*}\right)$ where the linearized system in closed loop remains stable. A good approximation of the value $m^{*}$ could be determined from the linearized system and the criterion of Routh. Using Matlab, we have estimated $m^{*}$ through the direct calculation of the eigenvalues of the state matrix $A$ of the linearized closed-loop system, and using a method of binary search. This has produced the estimated value $m^{*}=11.584$. To verify the claim that the robustness of the SFA approximates the robustness of the SF for small values of $h$, we have made several simulations for various values of reset period $h$ choosing a value of $m$ that was less than $m^{*}(m=10.5)$ and then setting a value of $m$ nearest to $m^{*}(m=10.9)$. The presented comparisons only regards the values of $x_{1}(t)$, because the other state variables have exhibited similar qualitative behaviors.

\subsubsection{Analysis for $m=10.5$}

- Taking $h=0.01$ : The differences between the results of the SF and SFA are very small (see Fig. 18).

- Taking $h=0.1$ : The differences between the results of the SF and SFA for $h=0.1$ are greater than the ones of $h=$ 0.01 , but are still very small, almost not distinguishable in Fig. 19. 


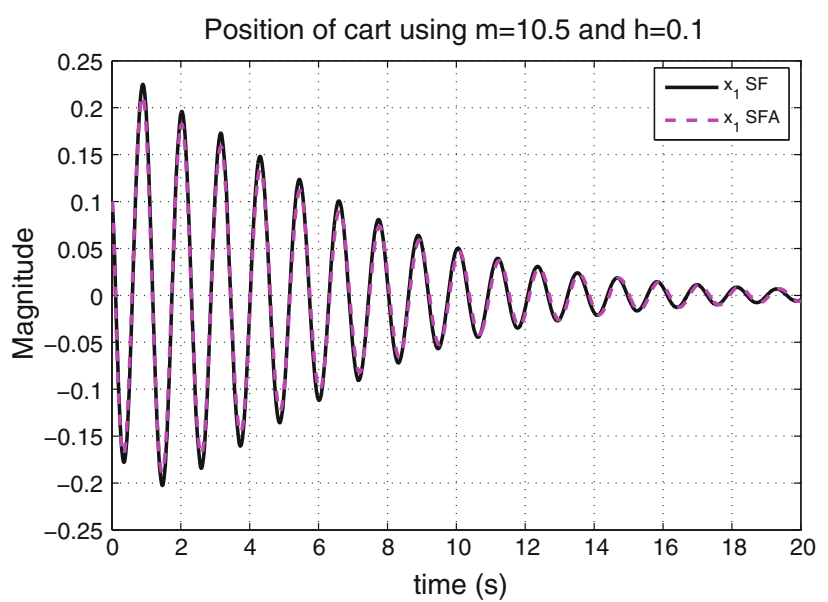

Fig. 19 Comparison of positions for SF and SFA with $m=10.5$ and $h=0.1$

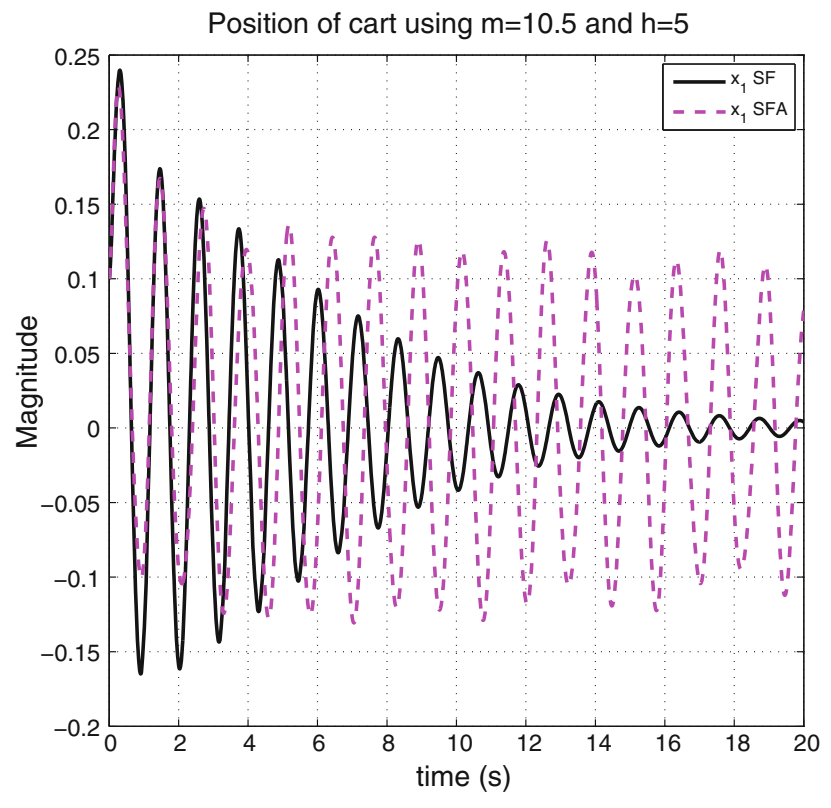

Fig. 20 Comparison of positions for SF and SFA with $m=10.5$ and $h=5$

- Taking $h=5$ : The robustness of SFA does not imitate the one of SF any more, as can it can be observed in Fig. 20. The difference between SF and SFA is now clearly visible in these figures. The oscillation is not damped in closed loop for the SFA, showing that robustness of SF is significatively greater than the SFA for this value $h$.

\subsubsection{Analisys for $m=10.9$}

Figure 21 shows the behavior of the actual states given by the two control laws studied SF and SFA in the case where $m=10.9$ with $h=0.01$. In this case, both closed-loop systems are unstable.

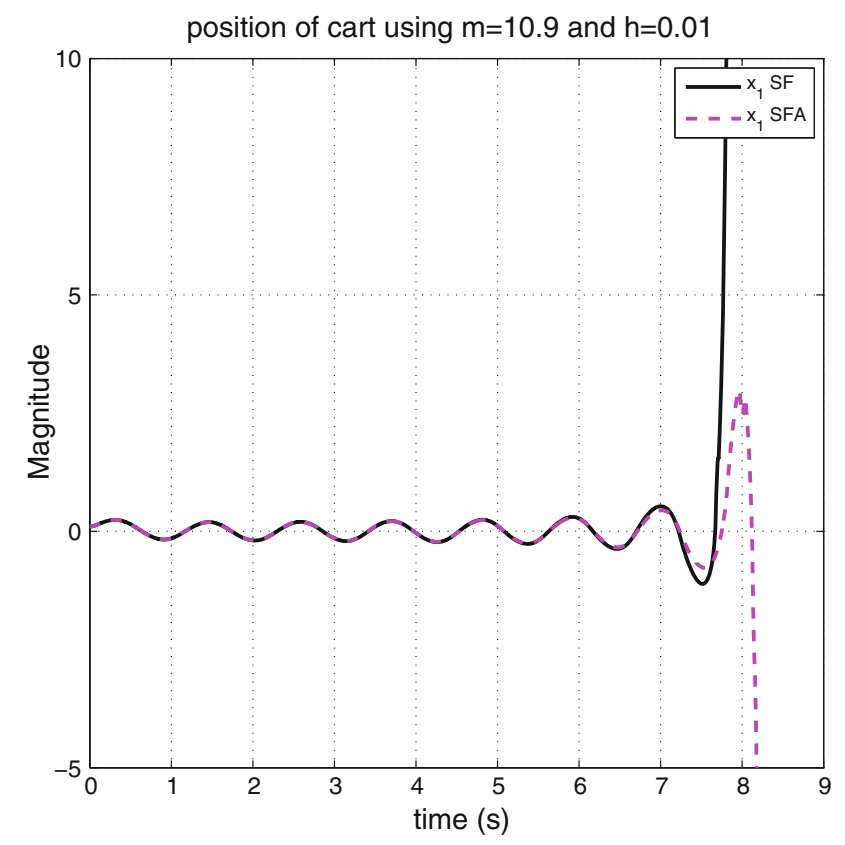

Fig. 21 Comparison of positions between SF and SFA with $m=10.9$ and $h=0.01$

The explanation for obtaining such instability for values slightly smaller than $m^{*}$ (both for SF and SFA) lies in the fact that the estimate obtained for $m^{*}$ refers to the linearized system, while the simulations have considered the nonlinear model of the pendulum.

\section{Conclusions}

The simulations in our case study have allowed to establish a behavior pattern described below.

(A) Performance of the Controller in Closed Loop In the absence of noise, the increase in $h$ degrades the controller performance (its transient behavior), reaching instability in closed loop for very large values for $h$ (vide Murgueytio 2011). However, the degradation of the estimation is much more noted by increasing $h$ when compared with the performance degradation so obtained. There is a big range of $h$ where the performance is still acceptable, but the estimate is already severely degraded in terms of accuracy.

(B) Noise Immunity (of the Actual State) In the presence of noise, one notes a degradation of the actual behavior of state with the increase in $h$, since the estimation in open loop has the same trend and causes degradation of the closed-loop system as a whole. It is observed that, as the mechanical system behaves as a low-pass filter, degradation of estimated state is really greater than the degradation of the actual behavior of the state. 
Table 1 Results of simulations of state feedback

\begin{tabular}{|c|c|c|}
\hline & Increase in $h$ & Decrease in $h$ \\
\hline $\begin{array}{l}\text { (A) Performance of the } \\
\text { controller in closed loop }\end{array}$ & Worsens with increase in $h$ & $\begin{array}{l}\text { Improves with decreasing } \\
\text { of } h\end{array}$ \\
\hline $\begin{array}{l}\text { (B) Noise immunity (real } \\
\text { state) }\end{array}$ & $\begin{array}{l}\text { The immunity decreases } \\
\text { with increase in } h\end{array}$ & $\begin{array}{l}\text { Increases, but is much less } \\
\text { sensitive than the } \\
\text { estimation }\end{array}$ \\
\hline (C) Robustness of stability & $\begin{array}{l}\text { The increase in } h \text { degrades } \\
\text { the robustness }\end{array}$ & $\begin{array}{l}\text { The reduction in } h \text { improves } \\
\text { the robustness }\end{array}$ \\
\hline
\end{tabular}

(C) Parametric Robustness of Stability For small values of $h$, it is found that the robustness of the stability of the system is very close to the robustness of perfect state feedback. The increase in $h$ degrades robustness of the system. Estimated state feedback for large values of $h$ becomes less robust than perfect state feedback.

We have summarized the qualitative results in Table 1.

It is important to point out that the estimation accuracy may be controlled by the value of the reset period $h$. In fact, this is a consequence of the results of Sect. 3.1 that shows that the estimation error tends to zero when $h$ tends to zero. Unlike traditional observers, whose estimate is asymptotic, the algebraic estimator provides a virtually fast estimate that is virtually exact. Also very important is that the proof of the Theorem 1 (and also of Theorem 2) consider a dynamical system of order equal to the original system. We believe that this aspect is important, ensuring itself a robust nature of the result so obtained. Note that any given estimator of the output derivative that obeys (19) will present the same closed-loop properties, since this is the only property of the estimator that was used to prove the stability result. Although the Theorems 1 and 2 are similar to the principle of separation, the proof of this result has not the same nature of traditional statements of separation theorems, since the dynamics of the estimator is not taken into account, but only the estimating error. Comparing the proof of the Theorem 1 (and 2) with the statements of the traditional separation theorem in its various versions (see for example Atassi and Khalil 1999, 2000, 2001), we have noticed a fundamental philosophical difference. In fact, the closed-loop system is modeled in general as an augmented system that contains the dynamic of original system, as well as the dynamic of estimator. Note that the estimate of the asymptotic observer converges to the actual state, while the algebraic estimator is virtually instantaneous. This extreme speed of estimation implies that there is no need to consider the convergence of the estimator in the proof of closed-loop stability, and this explains why the internal variables of the estimator are "transparent" to the stability proof.

It should be emphasized that the formula (19) is valid in the absence of noise, and another analysis should be done by a stochastic approach to ensure stability in some sense that is compatible to a noisy context. In this case, the analysis tools will have to take into account the nondifferentiability of the signals involved, for example using stochastic calculus.

Note that there is not a theoretical rule for adjusting an appropriate value of the reset period $h$, taking into account the compromise between performance and robustness, both on one side and rejection of noise on the other side. Another interesting research topic could consider this problem via the optimization of some reasonable criterium.

It is interesting to underline that the implementation of the algebraic estimators developed in recent literature is distinct from the implementation reported here (Mboup et al. 2009). In fact, a formula similar to (11) could be obtained in a way that the integration is made in the interval $[t-h, t]$, where $t$ is the current instant. In this context, there would be no need of re-initializing the estimators, whereas the error and rejection to noise of the filter cease to be variable in time, making the response more homogeneous. We believe, however, that the qualitative results and error analysis for these filters are quite similar to those results presented here.

The results reported here are analogous to the results of Ahrens and Khalil (2009), regarding a version of separation theorem using a switched high gain observer. There are also connections to the results of Levant (2003) using differentiation via sliding mode techniques. The compromise between rejection to noise, robustness and accuracy of the estimator here reported has paralleled the results of the previously mentioned studies. A comparison with these other approaches will be the subject of future research.

Finally, case studies reported in Murgueytio (2011) show promising results of algebraic estimators in closed loop with control laws based on flatness (flatness based control).

\section{Appendix: Proof of Theorems 1 and 2}

We begin this appendix with considerations that are common to the statements of Theorems 1 and 2. Note that the closedloop system with the feedback of the estimated state is of the form

$$
\begin{aligned}
\dot{y}(t)= & y^{(1)}(t), \\
\dot{y}^{(1)}(t)= & F\left(t, y(t), y^{(1)}(t)\right) \\
& +G\left(t, y(t), y^{(1)}(t)\right) \alpha(t, y(t), \hat{\dot{y}}(t)),
\end{aligned}
$$


where $\hat{\dot{y}}$ is obtained from (12) with the policy $h$-periodic re-initialization. The proof of stability will consider that the map $\hat{\dot{y}}(t)$ is a function of time that the order of the closedloop system studied is the same order of the original system. The dynamics of the estimator will be considered only to demonstrate the global existence and uniqueness of solutions (see remark at the end of the proof of Theorem 1).

Essentially, the proof considers (33) that is the closed-loop system with the algebraic estimator (that is, system SFA) as an approximation of the closed-loop system with a stabilizing state feedback (that is, system SF). In other words, the system (33) is the system SF changed by an uncertainty, which is limited by the estimation error. Thus, the proof reduces to show that the actual state of (33) is convergent to the equilibrium point. This is done via the same Lyapunov function of the system SF, by showing that it remains negative even in the presence of the estimation error. To show this, note that, by (33b) and (19), we have:

$$
\begin{aligned}
\|e(t)\| & =\left\|y^{(1)}(t)-\hat{\dot{y}}(t)\right\|, \\
& \leq h L \mid F(t, x)+G(t, x) \alpha(t, y, \hat{\dot{y}}) \|,
\end{aligned}
$$

where $L=7 / 3$. From the triangle inequality, it follows that

$$
\begin{aligned}
\|e(t)\| \leq & h L\left\|F+G \alpha\left(t, y, y^{(1)}\right)\right\|, \\
& +h L\left\|G\left[\alpha(t, y, \hat{\dot{y}})-\alpha\left(t, y, y^{(1)}\right)\right]\right\|
\end{aligned}
$$

and using the map $P(t, x)$ defined in the Hypothesis H1, the Lipschitz property of $\alpha$, and also the fact that the norm of $G$ is uniformly bounded, we have:

$$
\begin{aligned}
\|e(t)\| & \leq h L\|P(t, x)\|+h L \gamma K\|e(t)\|, \\
& \leq h L \delta\|x\|+h L \gamma K\|e(t)\| .
\end{aligned}
$$

Then, if $h$ is small enough in a way that $h L \gamma K<1$, then

$\|e(t)\| \leq h \mu\|x\|$,

where $\mu=L \delta /(1-h L \gamma K)>0$.

Using the notations of (21), (24), (25) and (27), we can rewrite (33) in the form

$$
\begin{aligned}
\dot{x} & =f(t, x)+g(t, x)[\alpha(t, \hat{x})], \\
& =f(t, x)+g(t, x) \alpha(t, x)+g[\alpha(t, \hat{x})-\alpha(t, x)] .
\end{aligned}
$$

And denoting

$\theta(t, x)=f(t, x)+g(t, x) \alpha(t, x)$,

we can rewrite the closed-loop system as

$\dot{x}(t)=\theta(t, x(t))+g[\alpha(t, \hat{x})-\alpha(t, x)]$,

where $\hat{x}$ é definido por (25). If $V(t, x)$ is a strict Lyapunov function for the system, we get:

$\dot{V}=\frac{\partial V}{\partial t}+\frac{\partial V}{\partial x} \theta(t, x)+\frac{\partial V}{\partial x} g[\alpha(t, \hat{x})-\alpha(t, x)]$.
Let

$\phi=-\left(\frac{\partial V}{\partial t}+\frac{\partial V}{\partial x} \theta(t, x)\right)$.

As $\| G \mid \leq \gamma$, by (21c), it follows that $\|g\| \leq \gamma$. Therefore, the Lipschitz property of $\alpha$ gives:

$\dot{V} \leq-\phi+\gamma K\left\|\frac{\partial V}{\partial x}\right\|\|e\|$.

We can then deduce from (34) that

$\dot{V} \leq-\phi+h \gamma K \mu\left\|\frac{\partial V}{\partial x}\right\|\|x\|$.

Proof of Theorem 1

Before presenting the proof, we state the following converse Lyapunov theorem:

Theorem 3 (Khalil 1996, Teo. 3.12) Let

$\dot{x}(t)=\theta(t, x(t))$

be system, where the map $\theta: \mathbb{R} \times \mathbb{R}^{n} \rightarrow \mathbb{R}^{n}$ is of class $C^{1}$, possessing an equilibrium point at the origin, which is globally exponentially stable. Assume that $\frac{\partial \theta}{\partial x}$ is uniformly bounded. Then, there exist positive constants $c_{1}, c_{2}, c_{3}$, e $c_{4}$, and a $C^{1}$ Lyapunov function $V: \mathbb{R} \times \mathbb{R}^{n} \rightarrow \mathbb{R}$ such that

- $c_{1}\|x\|^{2} \leq V(t, x) \leq c_{2}\|x\|^{2}$.

- $\dot{V}=\frac{\partial V}{\partial t}+\frac{\partial V}{\partial x} \theta(t, x) \leq-c_{3}\|x\|^{2}$.

- $\left\|\frac{\partial V}{\partial x}\right\| \leq c_{4}\|x\|$.

To begin the proof of Theorem 1, note that if

$\theta=\left[\begin{array}{c}y^{(1)} \\ P(t, x)\end{array}\right]$,

then

$\frac{\partial \theta}{\partial x}=\left[\begin{array}{c}U \\ \frac{\partial P}{\partial x}\end{array}\right]$

where

$U=\left[\begin{array}{ll}0 & I_{m}\end{array}\right]$

Therefore, as $\left\|\frac{\partial P}{\partial x}\right\|$ is uniformly bounded (see Hypothesis H1), we must have that $\frac{\partial \theta}{\partial x}$ is also uniformly bounded. We use the Lyapunov function $V$ whose existence is guaranteed by Theorem 3. From (37) and (38), it follows that

$\dot{V} \leq-c_{3}\|x\|^{2}+h \gamma K \mu c_{4}\|x\|^{2}$,

thus, for $h$ small enough, we get $N=c_{3}-h \gamma K \mu c_{4}>0$ and so

$\dot{V} \leq-N\|x\|^{2}$. 
This last condition combined with the first two conditions of the converse Lyapunov theorem proves the global exponential stability of the system in the closed loop with feedback of the estimated state (see Corollary 3.4 of Khalil 1996).

Remark The local existence and uniqueness of the solutions of the system are guaranteed by theorem 2.2 of Khalil (1996) applied to the system (12)-(33) between the moments of reset. Indeed (12) can be implemented by two differential equations of the form:

$$
\begin{aligned}
& \dot{z}(t)=y(t), \\
& \hat{\dot{y}}(t)=\frac{1}{\left(t-t_{k}\right)^{2}}\left[-2 z(t)+2\left(t-t_{k}\right) y(t)\right],
\end{aligned}
$$

where $z\left(t_{k}\right)$ is $h$ periodically reset to zero. The filter that is active works within an interval of time such that $t-t_{k}$ is in $[h / 2, h]$. The fact that there is no escape phenomena in finite time is guaranteed by the fact that $V$ is decreasing [and hence the state $x(t)$ is bounded]. Next, as the norm of $y(t)$ is uniformly bounded by a real number $J$, this implies that the norm of the integral of $y(t)$ at intervals of finite length $T$ not exceed $T J$. In particular, the derivative estimate is also always bounded. Now, it is easy to see from (39b) that the fact that actual output $y(t)$ converges to zero also implies that the derivative estimate of both filters also converges to zero (at least considering only the intervals where each filter is active).

\section{Proof of Theorem 2}

By (38) and part (iii) of Hypothesis H3, we have:

$$
\dot{V} \leq-W_{3}+h \gamma K \mu\left\|\frac{\partial V}{\partial x}\right\|\|x\| .
$$

Now assume that $h<\epsilon /(\gamma K \mu)$, onde $\epsilon$ is defined in part (iv) of H3. It follows that

$$
\dot{V} \leq-W_{4} \text {. }
$$

In this last condition, from parts (i) and (ii) of the Hypothesis H3, and by Corollary 3.3 of Khalil (1996), it follows that the origin is globally uniformly asymptotically stable.

\section{References}

Ahrens, J., \& Khalil, H. (2009). High-gain observers in the presence of measurement noise: A switched-gain approach. Automatica, 45 , 936-943.

Atassi, A., \& Khalil, H. (1999). A separation principle for the stabilization of a class of nonlinear systems. IEEE Transactions on Automatic Control, 44, 1672-1687.

Atassi, A., \& Khalil, H. (2000). Separation results for the stabilization of nonlinear systems using different high-gain observer designs. Systems \& Control Letters, 39, 183-191.

Atassi, A., \& Khalil, H. (2001). A separation principle for the control of a class of nonlinear systems. IEEE Transactions on Automatic Control, 46, 742-746.
Chen, C., \& Lee, J. (1995). Design of high-order digital differentiators using $l_{1}$ error criteria. IEEE Transactions on Circuits and Systems, 42(4), 287-291.

Chen, C. T. (1999). Linear system theory and design (3rd ed.). New York: Oxford University Press.

Craig, J. J. (1982). Introduction to robotics. Rio de Janeiro: PrenticeHall do Brasil.

Cunha, J. P. V. S., Hsu, L., Costa, R. R., \& Lizarralde, F. (2005). Controle de sistemas lineares incertos por modos deslizantes e observador de alto ganho sem peaking. Revista Controle \& Automação (SBA), 16, $449-466$.

Dabroom, A., \& Khalil, H. (1999). Discrete-time implementation of high-gain observers for numerical differentiation. International Journal of Control, 72, 1523-1537.

Diop, S., \& Fliess, M. (1991). On nonlinear observability. In ECC'91 (pp. 152-157).

Diop, S., Grizzle, J. W., \& Chaplais, F. (2000). On numerical differentiation algorithms for nonlinear estimation. In IEEE CDC'2000.

Diop, S., Grizzle, J. W., Moraal, P. E., \& Stefanopoulou, A. (1994). Interpolation and numerical differentiation for observer desing. In 1994 American control conference, Baltimore (pp. 1329-1333).

Duncan, T., Mandl, P., \& Pasik-Duncan, P. (1996). Numerical differentiation and parameter estimation in higher-order linear stochastic systems. IEEE Transactions on Automatic Control, 41(322), 522532.

Fliess, M., \& Ramirez, S. (2004). Control via state estimations of some nonlinear systems. In Proceedings of the symposium on nonlinear control systems (NOLCOS'04). http://hal.inria.fr/inria-00001096

Fliess, M., \& Sira-Ramirez, H. (2004a). Control via state estimations of some nonlinear systems. IFAC Nolcos conference, Stutgart.

Fliess, M., \& Sira-Ramirez, H. (2004b). Reconstructeurs d'etats. Comptes Rendus de l'Academie des Sciences, 338, 91-96.

Ibrir, S., \& Diop, S. (2004). A numerical procedure for filtering and efficient high-order signal differentiation. International Journal of Applied Mathematics and Computer Science, 14, 201-208.

Khalil, H. K. (1996). Nonlinear systems (2nd ed.). Upper Saddle River, NJ: Prentice-Hall.

Levant, A. (2003). Higher-order sliding modes, differentiation and output-feedback control. International Journal of Control, 76, 924941.

Lima, E. L. (1981). Curso de Análise (Vol. 2). Rio de janeiro: IMPAProjeto Euclides.

Mboup, M., Join, C., \& Fliess, M. (2009). Numerical differentiation with annihilators in noisy environment. Numerical Algorithms, 50(4), 439-467.

Mikusinski, J. (1960). Operational calculus. Dordrecht: Elsevier.

Murgueytio, Z. V. L. (2011). Estimação algébrica aplicada aos sistemas de controle-um estudo de casos. Master's thesis, Escola Politécnica.

Novaes, C. E. B. (2010). Métodos de estimação de derivadas via cálculo operacional e aplicações a problemas de controle. Master's thesis, Escola Politécnica.

Novaes, C. E. B., \& Silva, P. S. P. (2009). On non-asymptotic state observers and their implementation in discrete time. DINCON'09, Bauru (pp. 45-53).

Oliveira, T. R., Peixoto, A. J., \& Hsu, L. (2010). Controle por realimentação de saída para sistemas incertos fortemente não-lineares. Revista Controle \& Automação (SBA), 21, 69-81.

Oliveira, T. R., Peixoto, A. J., \& Hsu, L. (2011). Controle por realimentação de saída e modos deslizantes via função de chaveamento periódica aplicado ao problema de busca extremal. Revista Controle \& Automação (SBA), 22, 412-424.

Peixoto, A. J., Oliveira, T. R., \& Hsu, L. (2011). Rastreamento global via controle por modos deslizantes e observador com ganho dinâmico. Revista Controle \& Automação (SBA), 22, 308-324. 
Plestan, F., \& Grizzle, J. W. (1999). Synthesis of nonlinear observers via structural analysis and numerical differentiation. 1999 European control conference, September.

Reger, J., Mai, P., \& Sira-Ramirez, H. (2006). Robust algebraic state estimation of chaotic systems. IEEE 2006 CCA/CACSD/ISIC, Munich (pp. 326-331).
Reger, J., Sira-Ramirez, H., \& Fliess, M. (2005). On non-asymptotic observation of nonliner systems. 44th IEEE conference on decision and control, Sevilla. 